$11-5-2020$

\title{
UNHCR and partner practices of community-based protection across sectors in the East and Horn of Africa and the Great Lakes Region
}

Charles Mballa

Josephine Ngebeh

Machtelt De Vriese

Katie Drew

Abigayil Parr

See next page for additional authors

Follow this and additional works at: https://knowledgecommons.popcouncil.org/departments_sbsr-rh How does access to this work benefit you? Let us know!

\section{Recommended Citation}

Mballa, Charles, Josephine Ngebeh, Machtelt De Vriese, Katie Drew, Abigayil Parr, and Chi-Chi Undie. 2020. "UNHCR and partner practices of community-based protection across sectors in the East and Horn of Africa and the Great Lakes Region." Nairobi: UNHCR and Population Council. 


\section{Authors}

Charles Mballa, Josephine Ngebeh, Machtelt De Vriese, Katie Drew, Abigayil Parr, and Chi-Chi Undie 


\section{UNHCR AND PARTNER PRACTICES OF}

\section{COMMUNITY-BASED PROTECTION ACROSS SECTORS IN THE EAST AND HORN OF AFRICA AND THE GREAT LAKES REGION}

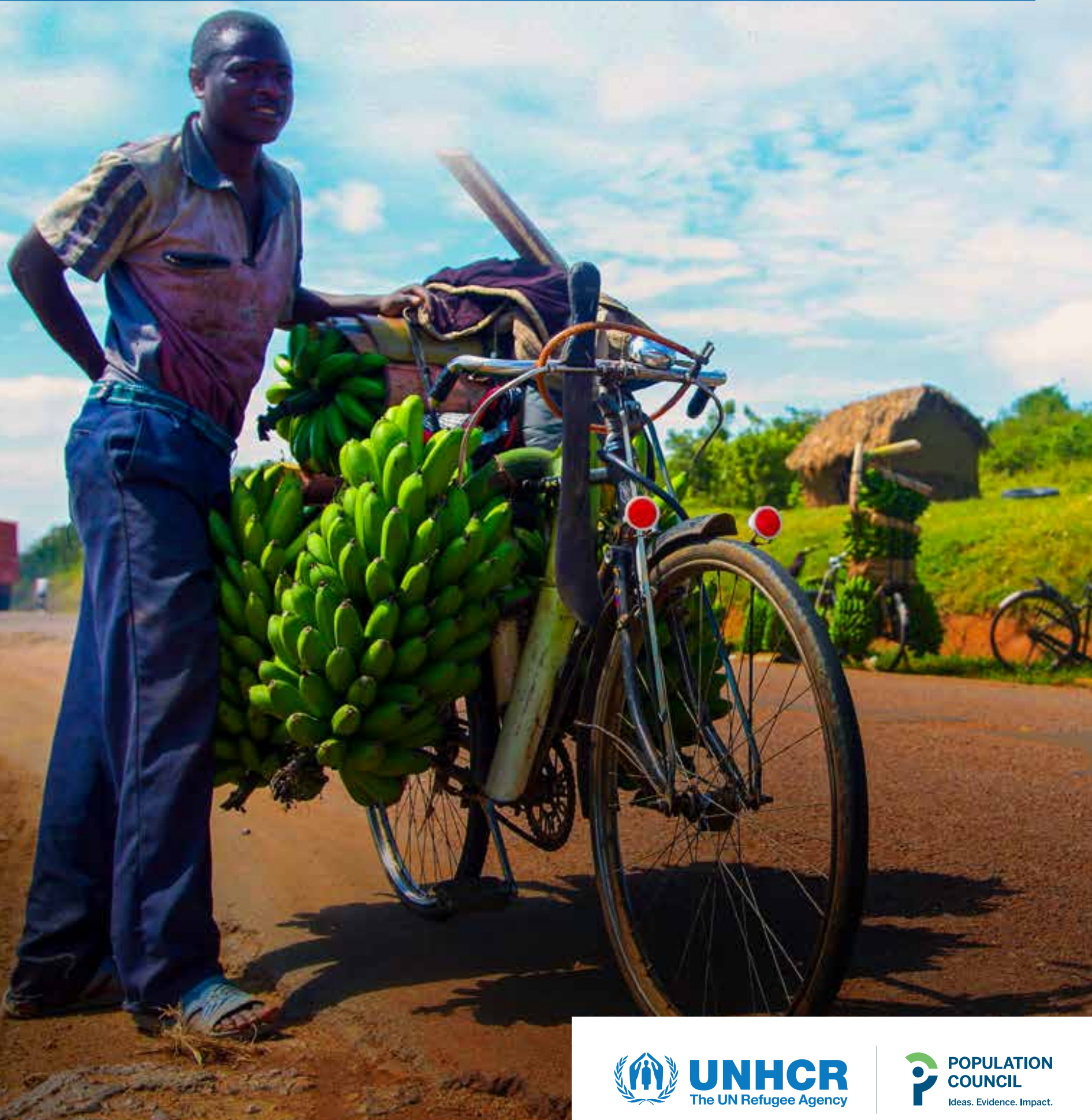




\section{SUGGESTED CITATION}

Charles Mballa, Josephine Ngebeh, Machtelt De

Vriese, Katie Drew, Abigayil Parr, Chi-Chi Undie. 2020.

UNHCR and Partner Practices of Community-Based

Protection across Sectors in the East and Horn of Africa

and the Great Lakes Region. UNHCR and

Population Council. 


\section{TABLE OF CONTENTS}

ACRONYMS

FOREWORD

INTRODUCTION

METHODOLOGY

HIGHLIGHTS OF PROMISING PRACTICES

COMPENDIUM OF PRACTICES

COVID-RELATED PRACTICES

9

1. CHILD PROTECTION AND YOUTH

9

2. COMMUNITY-BASED PROTECTION

3. SGBV/RISK MITIGATION/RESPONSE

26

NON-COVID-RELATED PRACTICES

28

1. CHILD PROTECTION AND YOUTH

28

2. COMMUNITY-BASED PROTECTION

35

3. GENDER EQUALITY

4. SGBV/RISK MITIGATION/RESPONSE 


\begin{tabular}{|c|c|}
\hline AGD & Age, Gender, and Diversity \\
\hline ARRA & Agency for Refugee and Returnee Affairs \\
\hline CBAC & Community-Based Adolescent Committee \\
\hline CBP & Community-Based Protection \\
\hline CCCT & Community Care Coalition Team \\
\hline $\mathrm{CHH}$ & Child-Headed Household \\
\hline CHW & Community Health Worker \\
\hline CORPS & Community Owned Resource Person \\
\hline CPC & Child Protection Committee \\
\hline CPV & Child Protection Volunteer \\
\hline CRRF & Comprehensive Refugee Response Framework \\
\hline CWC & Child Welfare Committee \\
\hline DICAC & Development and Inter-Church Aid Commission \\
\hline DRC & Danish Refugee Council \\
\hline DHR & Direction de l'Hydraulique Rurale \\
\hline EHAGL & East and Horn of Africa and the Great Lakes \\
\hline FDP & Food Distribution Points \\
\hline GE & Gender Equality \\
\hline HCW & Healthcare Workers \\
\hline HIAS & Hebrew Immigrant Aid Society \\
\hline IDP & Internally Displaced Person \\
\hline IPV & Intimate Partner Violence \\
\hline IRC & International Rescue Committee \\
\hline KAP & Knowledge, Attitudes, and Practices \\
\hline LWF & Lutheran World Federation \\
\hline MEARL & Monitoring, Evaluation, Accountability, Research, and Learning \\
\hline MHPSS & Mental Health and Psychosocial Support \\
\hline MINEMA & Ministry in Charge of Emergency Management \\
\hline MoA & Memorandum of Agreement \\
\hline MWC & Market Water Committee \\
\hline NGO & Non-Governmental Organizations \\
\hline NRC & Norwegian Refugee Council \\
\hline OPM & Office of the Prime Minister \\
\hline PPA & Project Partnership Agreement \\
\hline PRC & Post-Rape Care \\
\hline PTA & Parent-Teacher Associations \\
\hline RADO & Rehabilitation and Development Organization \\
\hline RCC & Refugee Central Committee \\
\hline RCCE & Risk Communication and Community Engagement \\
\hline RCW & Refugee Community Worker \\
\hline REF & Refugee Engagement Forum \\
\hline ROV & Refugee Outreach Volunteer \\
\hline SCl & Save the Children International \\
\hline SF & Stakeholder Forum \\
\hline SG & Steering Group \\
\hline SGBV & Sexual and Gender-Based Violence \\
\hline TB & Tuberculosis \\
\hline TdH & Terre des Hommes \\
\hline TWG & Technical Working Group \\
\hline UN & United Nations \\
\hline UNFPA & United Nations Population Fund \\
\hline UNHCR & United Nations High Commissioner for Refugees \\
\hline UNICEF & United Nations International Children's Emergency Fund \\
\hline VSLA & Voluntary Savings and Loan Association \\
\hline WASH & Water, Sanitation, and Hygiene \\
\hline WEO & Woreda Education Office \\
\hline WFP & World Food Program \\
\hline ZTVA & Zero Tolerance Village Alliance \\
\hline
\end{tabular}




\section{FOREWORD}

T

his compendium was jointly developed by UNHCR's Regional Bureau for the East and Horn of Africa and the Great Lakes, and the Population Council of Kenya. It collates a wide range of innovative community-based protection initiatives. The compendium is informed by a community of protection practice canvassed from partners, refugees, and host communities, as well as other stakeholders. The East and Horn of Africa and the Great Lakes region is host of 4 million refugees. This refugee population is typified by its diversity and includes over $80 \%$ of women, girls, boys, and adolescents with multiple protection needs.

In the spirit of the whole of society approach which underpins the Global Compact for Refugees, the compendium is the result of a longstanding partnership between the UNHCR Regional Bureau and the Population Council, Kenya, which began in 2014. The Regional Bureau and the Population Council, Kenya, collaborated, guided by a technical cooperation framework which aims to strengthen programming and information-sharing in refugee settings.

The compendium attests to the resolve shown by both institutions to operationalize foundational protection guidance, including: the Age, Gender, and Diversity Policy and Approaches (2018); the Accountability to Affected Populations Guidelines; the Inter-Agency Standing Committee Guidelines, Inclusion of Persons with Disabilities in Humanitarian Action, and other protection frameworks which reinforce the aims of the Global Compact for Refugees reaffirmed by Member States of the UN General Assembly. The compendium also seeks to vividly showcase multi-stakeholder approaches in pursuit of collective protection outcomes, and to outline age, gender and diversity sensitive prevention and responses to COVID-19 in a mutually reinforcing manner.

It is our sincere hope that this compendium will serve as practical tool for stakeholders to enhance protection delivery; promote direct participation of Persons of Concern and host communities in decision-making; and document innovative practices in a manner which tangibly and meaningfully impacts the lives and diverse protection needs of millions of refugees in the region.

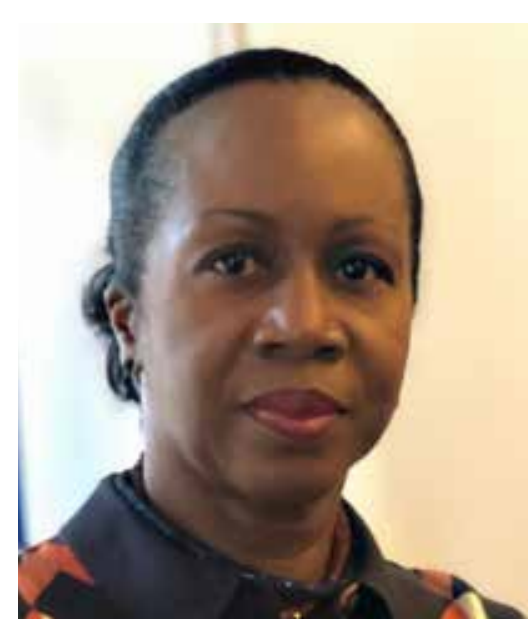

Clementine Awu Nkweta Salami Director, UNHCR Regional Bureau for East and Horn of Africa and the Great Lakes Region

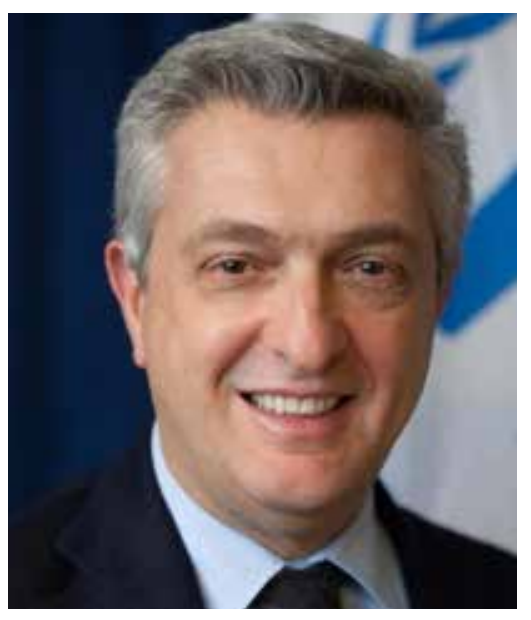

Filippo Grandi

UN High Commissioner for Refugees UNHCR

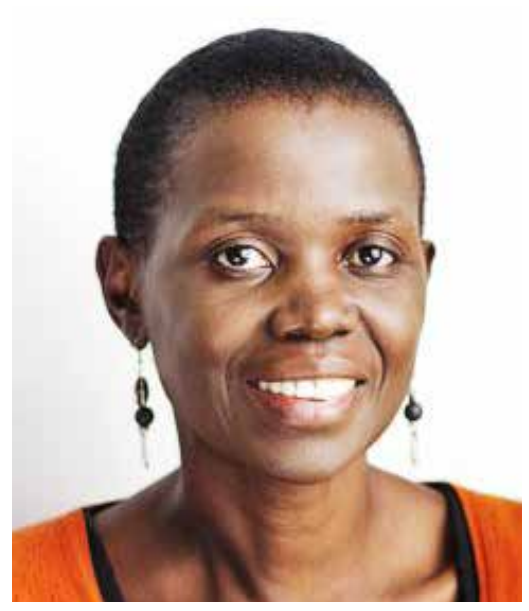

Harriet Birungi

Country Director \& Senior Associate Population Council, Kenya 


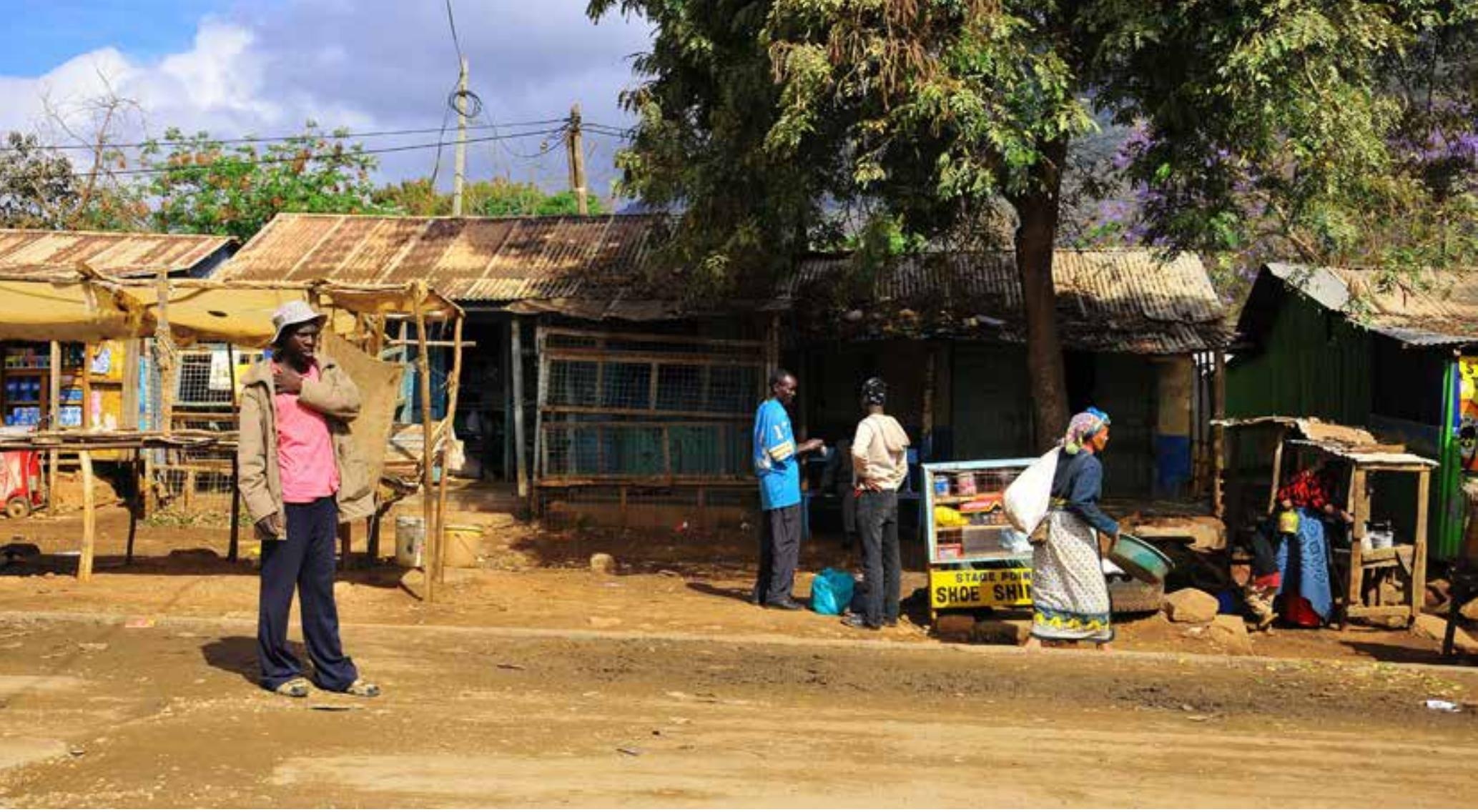

\section{INTRODUCTION}

This compendium summarizes regional information on promising practices, emerging practices, and case studies that form part of the response of the United Nations High Commissioner for Refugees (UNHCR) and its partners to the needs of refugees, internally displaced persons (IDPs), and host communities in the East and Horn of Africa and Great Lakes (EHAGL) region. The documentation of these practices serves several purposes. It fosters south-south and global exchange, learning, and capacity-building, while inspiring UNHCR and its partner program developers and implementers with fresh ideas for resolving common challenges.

The global COVID-19 pandemic makes this compendium even more critical at this time, as operations grapple with meeting the needs of Persons of Concern in the context of unprecedented lockdown and physical distancing regulations, which have radically changed service delivery mechanisms in refugee settings. Innovations borne out of these circumstances are important to capture and share across sectors and thematic areas. Not only can interventions applied successfully in one sector be employed to resolve issues in another, but the documentation of interventions permits a better understanding of programming strengths and gaps. It also illuminates common themes within and across country operations.

In addition to practices related to COVID-19, the compendium also documents interventions that preceded the pandemic, and that will therefore continue beyond COVID-19 as normalcy is restored. This approach helps ensure that innovative practices that were ongoing prior to COVID-19 are not overlooked - particularly given their importance as COVID-19 restrictions fluctuate and recede over time.

Despite concerted efforts (described in the next section) to make the compendium as inclusive and expansive as possible, it is important to note that this document is not representative of all practices occurring within UNHCR field operations by any means (as compendium submissions by UNHCR and partners were voluntary). Rather, it represents an important starting point for gaining insight into the vast range of efforts that provide emerging solutions to emerging challenges. The compendium is therefore a living document that recognizes the evolution of program solutions, and that will hopefully encourage more documentation and results generation to capture accomplishments, and the growth and maturation of practices that often go unnoticed.

The compendium showcases Persons of Concern' and host communities as bona fide partners of UNHCR, conceptualizing or co-conceptualizing many of the documented practices according to their emerging needs. It also highlights the creativity of these partners, along with that of UNHCR, governments, and UNHCR implementing agencies.

1 This includes refugees, asylum seekers, Internally Displaced People (IDP), returnees and statelesss persons. 


\section{METHODOLOGY}

The UNHCR Regional Bureau for the EHAGL Region oversees a total of 11 countries, namely: Burundi, Djibouti, Eritrea, Ethiopia, Kenya, Rwanda, Somalia, South Sudan, Sudan, Tanzania, and Uganda. UNHCR's Community-Based Protection (CBP) Unit invited all country operations to identify noteworthy practices pre- and during COVID-19. To facilitate the identification process, a UNHCR self-rating scale ${ }^{2}$ was made available to UNHCR Sector Focal Points, who were in turn asked to circulate the scale and the UNHCR methodology document for collating practices ${ }^{3}$ to their operations. Multi-functional teams were recommended as a mechanism for ensuring that the identification of practices was as inclusive as possible, and that Persons of Concern were engaged in the process, along with implementing partners. A UNHCR practice submission template 4 was also recommended as the format for operations to use in writing up and submitting their practices. In order to encourage submissions, these recommendations were not presented as prescriptions.

The UNHCR methodology document for collecting practices outlines three categories for classifying practices on the ground, namely, promising practices, emerging practices, and case studies. These categories are defined below.

\begin{tabular}{|c|c|}
\hline Category & Definition \\
\hline Promising Practice & $\begin{array}{l}\text { A practice that is proven to work well and produce sustainable results and has a protective } \\
\text { and/or transformative potential for individuals and/or communities, as demonstrated by } \\
\text { reliable results. It can serve as a model to be replicated and scaled up. }\end{array}$ \\
\hline Emerging Practice & $\begin{array}{l}\text { A practice that shows early indications of producing positive results to positively transform } \\
\text { lives of individuals and/or communities. }\end{array}$ \\
\hline Case Study & $\begin{array}{l}\text { A practice, or part of a practice, that adhered, or attempted to adhere (and faced challenges), } \\
\text { to defined criteria without requirement for provision of evidence or any judgement as to its } \\
\text { results or sustainability. It can provide insights and lessons learned into future programming } \\
\text { even when it does not meet certain aspects of criteria. }\end{array}$ \\
\hline
\end{tabular}

As outlined in the UNHCR methodology document,

'Promising practices are those that meet higher standards and demonstrate evidence, and they will be subject to more scrutiny. Emerging practices are those that show some signs or potential of producing results. ... [C] ]ase studies are more loosely defined as practices that meet basic requirements and show some signs of or have potential for producing results. They may be at different stages within the programming cycle with varying degree or quality of evidence of results. [Emerging practices and case studies] are both worth documenting, as they can inform future programming.5

Two virtual meetings were held with UNHCR Sector Focal Points to clarify the purpose of the compendium, reinforce its importance, and increase country operation submissions.

2 UNHCR (2019). Methodology and Background Document: Collecting Practices and Case Studies - Child Protection, Community-Based Protection, Gender Equality, Sexual and Gender-Base Violence, pp. 12-18.

3 Ibid.

4 Ibid., pp. 16-17.

5 UNHCR (2019). Methodology and Background Document: Collecting Practices and Case Studies - Child Protection, Community-Based Protection, Gender Equality, Sexual and Gender-Base Violence, p. 7. 
In September 2020, a three-person review team ${ }^{6}$ independently assessed each submission, categorizing each one according to its alignment with the above definitions of 'promising practice,"emerging practice,' and 'case study.' For cases in which reviewers' assessments were not similar, the assessment given by the majority of reviewers (2 out of 3) was used as the final categorization. Where conflicts of interest arose (due to reviewers' affiliation/involvement with a practice under assessment), the reviewers concerned abstained from participating in the assessment in question. Furthermore, three virtual regional workshops were convened in September 2020, bringing together country operations to discuss their submissions, give presentations on selected practices, reinforce submitters' understanding of the three practice categorizations, and come to consensus on the final categorization assigned by assessors. The first regional workshop was meant to focus on any practices submitted from Eritrea, Djibouti, Somalia, South Sudan, and Sudan; while the second centered on Ethiopia, Kenya, and Uganda; and the third workshop brought together Burundi, Rwanda, and Tanzania. Following the workshops, submitters were given several days to submit additional information about any submitted practice, if there was existing evidence which had not been outlined in the submission template, but could help inform the final categorization of the practice. The three virtual regional workshops brought together a combined total of 120 participants.

All submissions were considered, whether the submission template was employed for the submission of practices or not (the only exceptions were submissions that attempted to capture practices in 1 to 2 sentences, and therefore did not provide sufficient information). While this posed a challenge for presenting practices in a uniform manner, it expanded participation and ensured the inclusion of innovative practices from across the region: The compendium comprises nearly 70 practices from 10 country operations.

The compendium is organized into two main sections: COVID-19-related practices, and non-COVID-19-related practices. Each main section is further organized into UNHCR thematic areas, namely: Child Protection and Youth, Community-Based Protection, Gender Equality, and Sexual and Gender-Based Violence (SGBV)/Risk Mitigation/Response. It is noteworthy that Accountability to Affected People cuts across the thematic areas reflected in this compendium.

Each practice is described, with an outline of results also included for Promising Practices, and, frequently, for Emerging Practices. For the sake of brevity, the practice descriptions do not delve into issues such as the challenges experienced in implementation, but attempt to supply sufficient information for the reader to understand the goals and components of each intervention. Contact information is then provided to allow readers to follow-up directly with those who conceptualized the practices and/or are implementing them first-hand. Indeed, it is hoped that such interactions will occur within and across country operations, and that the compendium will continually foster information-seeking and sharing.

The overlap between thematic areas is noteworthy, and results in practice categorizations that are not necessarily flawless across the board. Nonetheless, this reality demonstrates the interconnectedness of thematic areas, which is appropriate.

6 Two Innovation Officers, with the UNHCR EHAGL Bureau, and with the Innovation Service (Global), respectively; and a Population Council Senior Associate. All reviewers were based in Nairobi, Kenya. 


\section{HIGHLIGHTS OF PROMISING PRACTICES}

T his introductory section of the compendium ends with highlights from the promising practices identified in the region based on voluntary submissions from stakeholders. It focuses on the results or impacts generated by these practices. A more detailed account of each practice submitted for inclusion in the compendium (including all promising practices) is laid out in the remainder of the document.

\section{Promising Practice from Ethiopia: Education Assessment and Programming and the Community Engagement Approach}

\section{Results}

- Since adoption of the approach in 2015, it has led to improved attendance and retention at school level.

- There was 83\% attendance at the primary school level in 2019 and a 53\% increase in enrolment at secondary school level in refugee and host community schools combined in the first half of 2020.

\section{Promising Practice from Rwanda: Community Hygiene Work Group and the Community's Joint Effort in COVID-19 Prevention, Including Local Musicians}

\section{Results}

- As part of joint COVID-19 prevention actions, the refugee community has built 122 additional hand washing stands to promote hygiene in the camp.

- Findings from a Knowledge, Attitudes, and Practices (KAP) survey indicated that COVID-19 related knowledge in the camp was generally high, and that the majority of refugees in the camp concerned recognize communitybased health communication a significant source of information. Furthermore, refugees scored higher in KAP related to handwashing (compared to other hygiene behaviors), partially reflecting the positive impact of the joint community effort in establishing handwashing stands.

- Women scored lower than men on COVID-19 related knowledge, due to illiteracy and limited access to phone/ radio.

\section{Promising Practice from Rwanda: Voluntary Savings and Loan Associations (VSLAs)}

\section{Results}

- Mahama Refugee Camp currently has 15 VSLAs mainly formed by Persons with Disabilities, although participation is open to all community members.

- Community members, including leaders, have joined income generating associations initiated by Persons with Disabilities. All VSLAs combined are composed of with 361 community members (159 male, 202 female). Of these, 90 are Persons with Disabilities.

- A total of 15 VSLAs have opened-up bank accounts.

- VSLAs are now sought out by various stakeholders (e.g., the Rwandan government and foreign embassies) to participate in camp competitions, exhibitions, and award ceremonies, and other special occasions. During mass meetings and various International Days, time slots are allocated to refugees with disabilities, their families, and partners to share testimonies about the life-changing effects of VSLAs.

- Communities testify that refugees with disabilities also have the capacity and strength to support their families and community at large.

\section{Promising Practice from Rwanda: Survivor Identification and Response through Screening}

\section{Results}

- In 2017, participating health facilities in 2 Rwanda camps experienced a 300\% increase in the proportion of survivors identified and connected to comprehensive SGBV care within 2 months after the commencement of the screening intervention.

- In 2019, in the 5 participating refugee camps in Rwanda, 326 persons were identified as SGBV-positive via screening, and 276 were referred to SGBV response services. 
- In 2019, participating health facilities in refugee camps in 4 countries combined (including Rwanda) experienced a $129 \%$ increase in the proportion of survivors identified and connected to comprehensive SGBV care within 6 months after the commencement of the screening intervention.

- In the 5 Rwanda camps where routine SGBV screening is being conducted, SGBV response partners report a sudden increase in cases received, which is thought to relate to the significant increase in SGBV survivor detection and access to care. Qualitative data collected from both clients and providers at the health center indicate that women and girls are now aware that, in addition to the SGBV response partner/agency, they can also report SGBV incidents at the health center. This has contributed to the number of detected cases of SGBV.

- In addition to Rwanda, the intervention has been replicated by UNHCR and its implementing partners in South Sudan, Uganda, and Zambia.

- In collaboration with the Population Council, UNHCR has conducted an initial set of meetings with education partners, district, teachers and students, to prepare the ground for introducing a child sexual abuse screening intervention in schools in Mahama, Mugombwa and Kigeme Camps. This initiative, also initially developed and evaluated in Kenya by the Population Council and Kenyatta National Hospital, is pending due to COVID19.

\section{Promising Practice from Uganda: The 'Zero Tolerance Village Alliance' (ZTVA)}

\section{Results}

- Evaluation results of this community-based SGBV prevention intervention demonstrated statistically significant reductions in the occurrence of: physical intimate partner violence (IPV) (for men and women), sexual IPV (for men), non-partner physical violence (for men and women), and non-partner sexual violence (for women).

- To date, the ZTVA intervention has been implemented in nearly 15 refugee villages in Uganda. Background preparations (including training of implementing partners, sensitization of community leaders, and/or creation of Stakeholder Forums, etc.) have also been carried out to eventually introduce the intervention in refugee settings in Djibouti, Ethiopia, and Zambia. 


\section{COMPENDIUM OF PRACTICES COVID-RELATED PRACTICES}

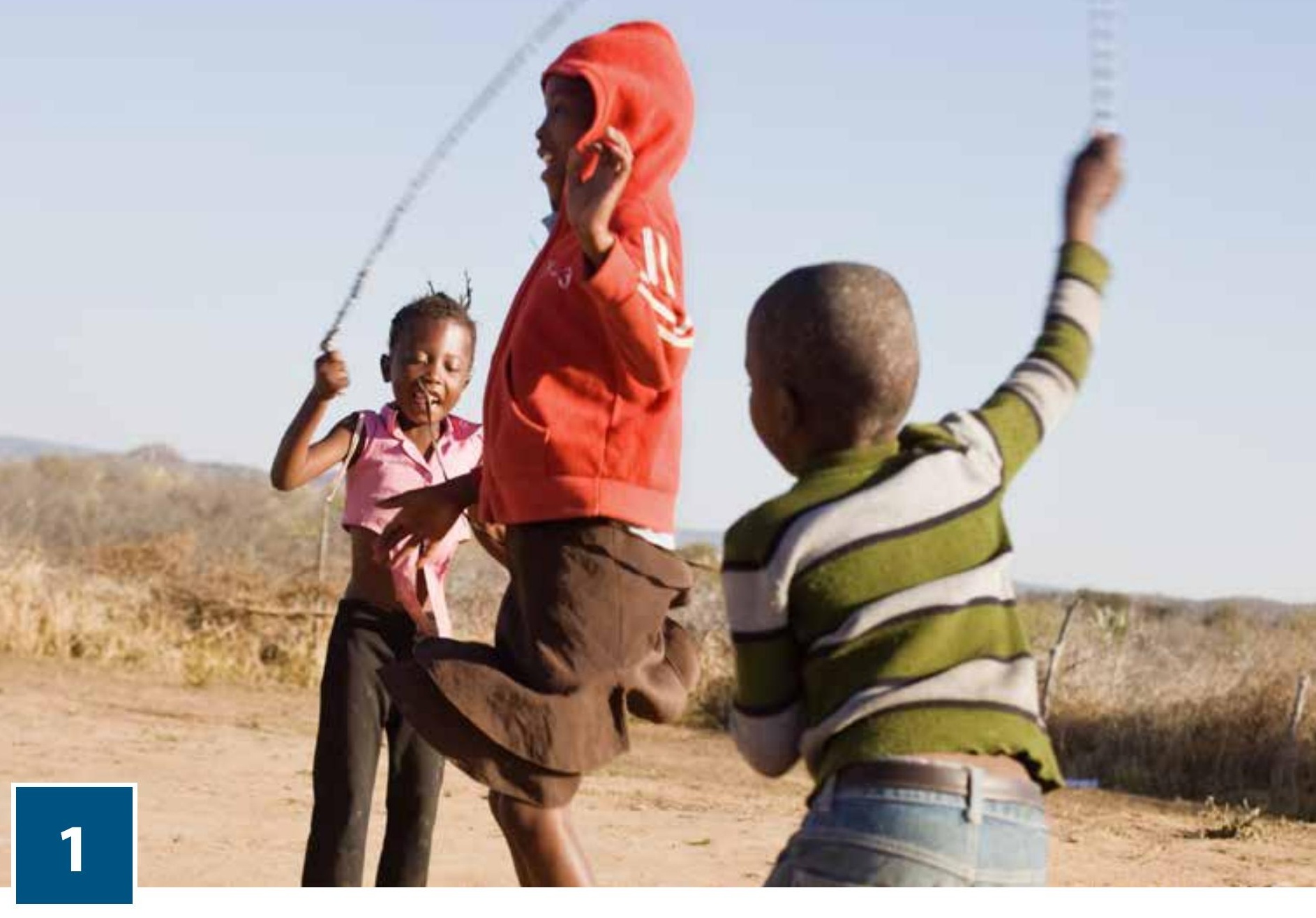

\section{CHILD PROTECTION AND YOUTH}

\section{Emerging Practice from Djibouti: Remote Child Protection Case Management Using Incentive Workers and Community-Based Protection (CBP) Members}

\footnotetext{
The detection of COVID-19 cases in refugee camps led to government-mandated lockdowns which prohibited entries into (and exits from) these locations by refugees, implementing partners, and UNHCR alike. This led to an unprecedented situation in which some children were separated from their parents (e.g., in cases where parents had already traveled outside the camps for work or medical care).

This emerging practice therefore entails UNHCR and its implementing partner collaborating remotely with incentive workers and CBP members working in the field of child protection in the refugee camps to monitor the individual case management of separated children, unaccompanied children, children at risk, and neglected or abandoned children.
} 
The specific components of this intervention are home visits, awareness-raising, and child protection case management data collection, as outlined below:

\section{Home visits}

- Assessment of children's needs and living situation in the refugee setting, including shelter, financial support, food assistance and medical assistance.

- Identification of children with specific needs and protection concerns, such as experience of violence (physical, sexual violence), early marriage, and pregnancy.

o Identification of neglected, abandoned, separated, unaccompanied, and orphan children.

\section{Awareness-raising}

- Raising community awareness of protection issues of concern, such as children's rights, child sexual and physical abuse, child labor, and early marriage.

o Raising community awareness of community-based COVID-19 protection measures.

\section{Data collection}

- Documenting the number of children within the camp that are separated, orphans, abandoned, or unaccompanied.

o Weekly reporting on the activities conducted the camp and current situation at the camp.

o Identifying child protection cases that need immediate assistance and reporting.

- Conducting a household survey to assess the psychosocial well-being of household members (including children) on the impact of COVID-19 restrictions on the daily lives of refugees.

\section{Results}

A total of 41 child survivors were identified via this intervention in the initial 4-month period after the onset of COVID-19. The survivors are enrolled in the ongoing case management program.

Partner(s): International Children's Action Network

For further information, contact:

Laura De Somer: desomer@unhcr.org

Arnaud Ntahompagaze: ntahompa@unhcr.org

Helene Chege Wangui: helen.wangui.c@gmail.com

\section{Emerging Practice from Tanzania: Community Radio for Education during COVID-19}

n refugee camps in Tanzania, a multifaceted approach is being employed to ensure the continuity of schooling during the COVID-19 pandemic. The approach involves the use of a distance learning program via community radio, coupled with the preparation of an education packet for delivery to households.

The Refugee Education Working Group held consultations with the government and obtained permission to produce and broadcast an education radio program that would meet refugees' needs. Once they had obtained permission, the EWG began working with Radio Kwizera on planning for the broadcast. The planning process has involved working through financial and technological constraints. As there is limited access to radio sets and other devices in the refugee camps, the Refugee Education Working Group is working to procure and distribute solar-powered radio devices to households. UNHCR education partners are also re-programming their budgets and approaches to accommodate emerging needs.

In addition, take-home education packages, including photocopies of textbooks based on the curriculum, are being prepared for home-based learning, targeting mainly secondary school students.

Partner(s):

Refugee Education Working Group

Radio Kwizera

For further information, contact:

Tamar Joanian: joanian@unhcr.org 


\section{Emerging Practice from Uganda: Child Ambassadors for COVID-19}

Thin

he COVID-19 pandemic necessitated the commencement of community education to ensure compliance with Ministry of Health directives, including handwashing, staying at home, and physical distancing. In response, World Vision began a community sensitization initiative in collaboration with community leaders, including Child Protection Committee members (and block leaders who distributed soap to vulnerable households for handwashing. In the process of household distribution, it was observed that children jested with the household distributors, yelling out the word'corona.' Among the household distribution team, 'William,' a South Sudanese refugee child, who happened to belong to a World Vision-initiated hygiene club in school, was the only member that piqued the interest of children, and that they seemed eager to listen to. Consequently, children became a vital part of the program. The importance of their involvement in the program was underscored by the lockdown context during the pandemic, which disrupted children's routines, leaving them idle with no place to go, unable to play and interact with friends. This placed new stressors on parents and caregivers, who often either had to forgo work, or find new childcare options.

This intervention aimed to empower children to effectively communicate with their peers on the new situation created by COVID-19, and to engage in adhering to preventive measures. The intervention began with William, the hygiene club member brought on board to provide support as an ambassador. His role was to create awareness among other children in the settlement, and he was provided with a megaphone to convey messages in various villages, sensitizing fellow children on COVID-19 prevention. Since then, 19 child ambassadors ( 9 female and 10 male) have participated in a 2-day COVID-19 training sessions carried out by two health workers and one staff member from an implementing partner organization that focuses on communication with communities. The training session builds children's capacity to disseminate COVD19-related information to their peers and communities. Like William, trained children are given megaphones to reach other children in their home areas. They are also provided with leaflets containing child-friendly messages crafted by the Ministry of Health, and internalized by the children as part of the training.

\section{Results}

By June 2020, 948 children (458 female and 490 male) had been reached with messages on COVID-19 by trained child ambassadors during home-to-home sensitization sessions. Through their participation in this initiative, children observed and reported a lack of soap in most families reached, and requested an additional soap distribution exercise to promote hygiene, with the support of Child Protection Committees.

Partner(s):

World Vision International

For further information, contact:

Veronique Njo: njo@unhcr.org

Brenda Adrako Madrara: Brenda_Madrara@wvi.org; abitimorenda@gmail.com 


\section{Case Study from South Sudan: Student Mobilization for COVID-19 Messaging in Urban Areas}

n Gorom refugee settlement in Juba, student beneficiaries of a UNHCR scholarship volunteered to assist their communities by sharing COVID-19 prevention information. The volunteers were given orientation on World Health Organization key prevention information and divided into teams led by community health workers or hygiene promoters. The teams conduct household-level information campaigns, demonstrating basic hygienic practices in line with recommended IEC materials. The student teams also refer individual medical cases to health partners.

Partner(s): various

For further information, contact:

Tamar Joanian: joanian@unhcr.org

\section{Emerging Practice from South Sudan: 'Everyone Can Make a Difference; Every Action Counts'}

n Doro refugee camp, each village has a youth representative (Chairperson) who participates in the camp's Youth Committee. Eight different ethnicities have been identified among the refugees, each with its own dialects. Since there is a need for sensitization on COVID-19 and good hygiene practices, this diversity serves well, enabling communication in the various local languages. To be successful, contextualization of key COVID-19 messages to the local camp context is required.

In this youth-led intervention, young people in the refugee camp have taken up COVID-19 community sensitization. Youth Chairpersons have collaboratively composed eight songs on COVID-19 in their local languages. The songs are based on COVID-19 messages that have been approved by the World Health Organization. This group of youth visit one community after another, sensitizing refugee community members about COVID-19, and singing the translated version of the songs according to the dialect spoken in the specific sub-location.

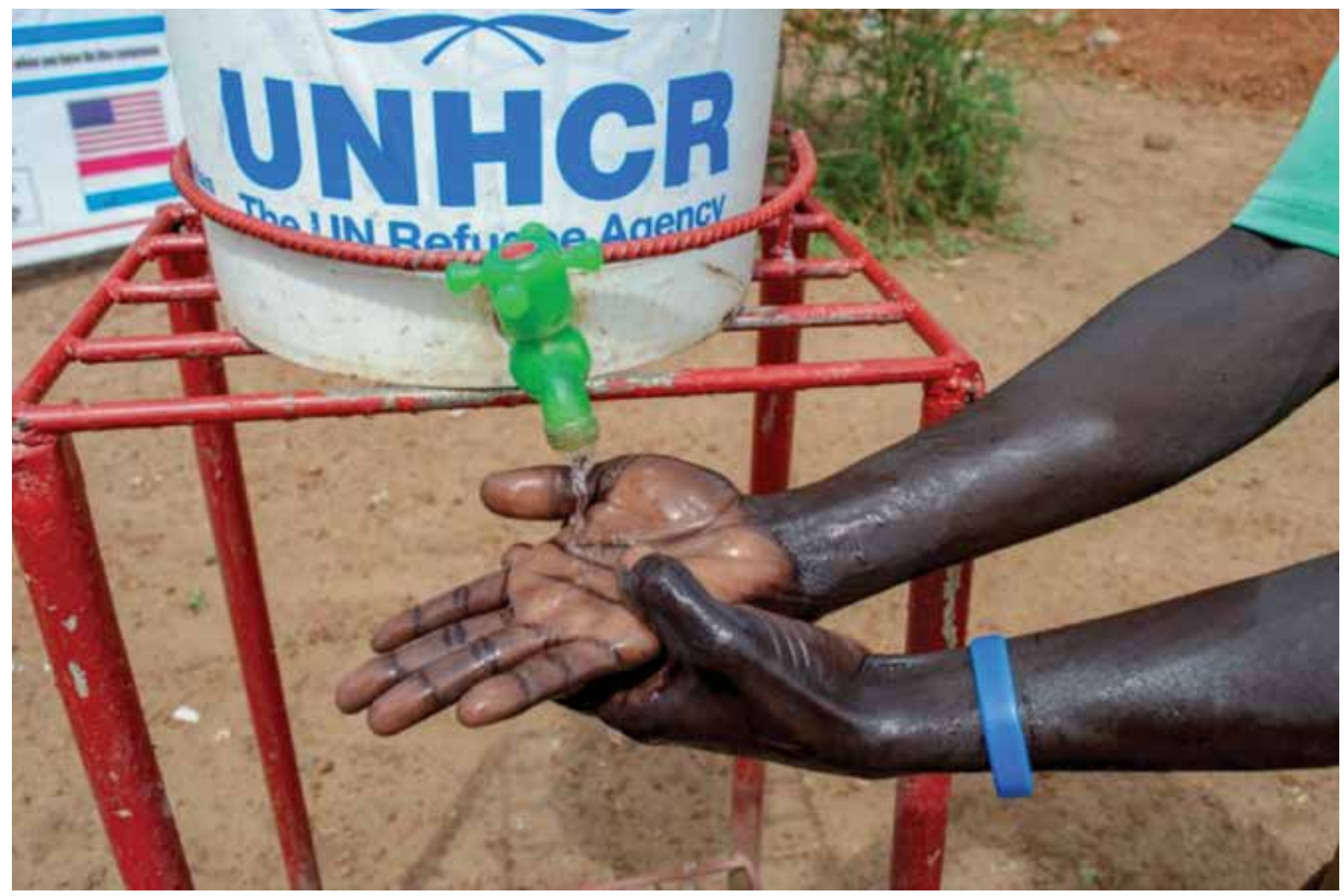



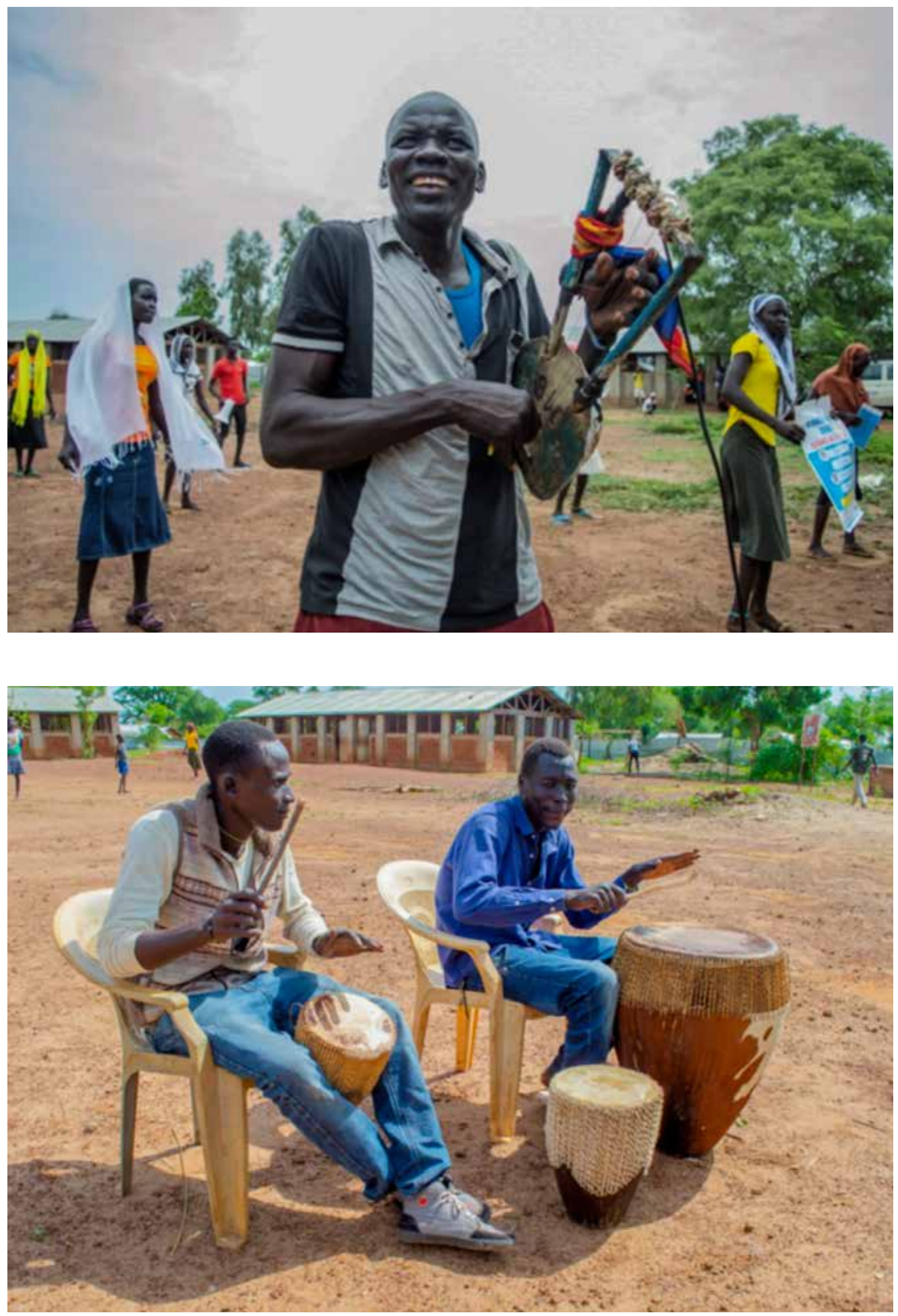


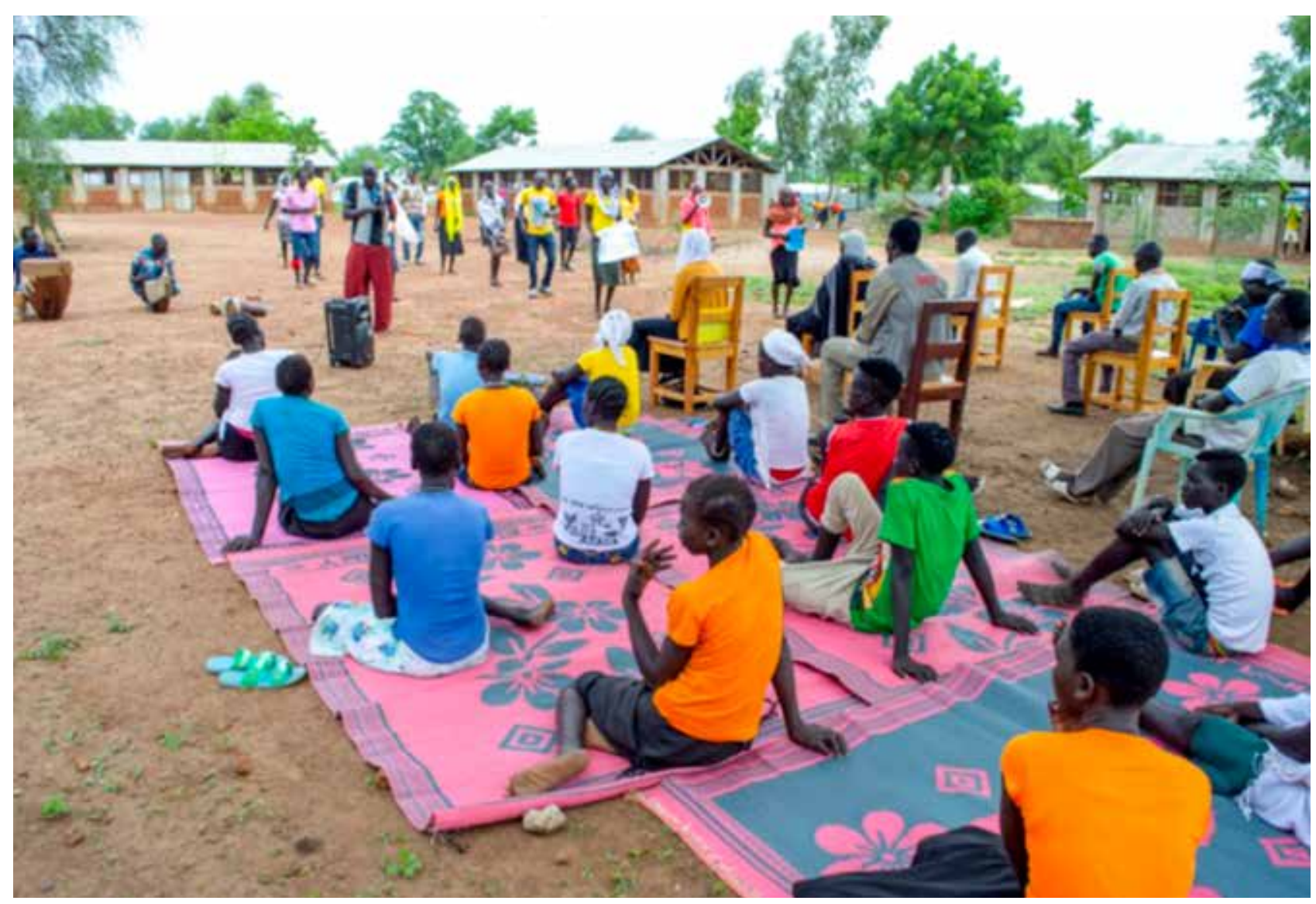

The group ensures physical distancing throughout their performances, along with proper handwashing.

During each event, a team of volunteers directs the audience to handwashing stations. A large banner with COVID-19 content is hung behind the handwashing stations. As the community members line up, each person is instructed to stand approximately one meter apart. To ensure that they maintain this distance, community members are encouraged to position themselves in the middle of circles that have been marked on the ground with sand. A volunteer is stationed adjacent to each handwashing station to instruct women, men, and children on recommended handwashing techniques.

Chairs for the audience are spread one meter apart and everyone who opts to sit on mats is encouraged to maintain the same distance. When all seats (both chairs and mats) are filled up, the show kicks off. The songs encompass local metaphors and highlight good practices, such as the avoidance of hand-shaking and physical distancing. After a series of songs, the group gives talks with COVID-19 prevention content.

The Youth Committee documents the number of areas visited, the number of performance session conducted, and the number of people reached. In July 2020, through a multisectoral approach under the SGBV program, community-based SGBV structures (Protection monitors, SASA! Activists, SGBV advocates) were engaged in awareness sessions and the dissemination of SGBV prevention messaging which were integrated with COVID-19 preventive messaging. A total of 4,899 community members (2,092 male and 2,807 female) were reached.

This youth group started the initiative by itself but needs the support of humanitarian actors to sustain its activities. Local initiatives such as this one can provide an impactful platform for conveying critical messages adjusted to the local context and customs.

\section{Partner(s):}

Commission for Refugee Affairs

Danish Refugee Council

\section{For further information, contact:}

Dr. Gebrewold Petros Yohannes: petros@unhcr.org

Thomas John Hither, Chairperson, Youth Committee, Doro Refugee Camp

Alor Kuol, DRC Deputy Camp Manager, Doro Refugee Camp 


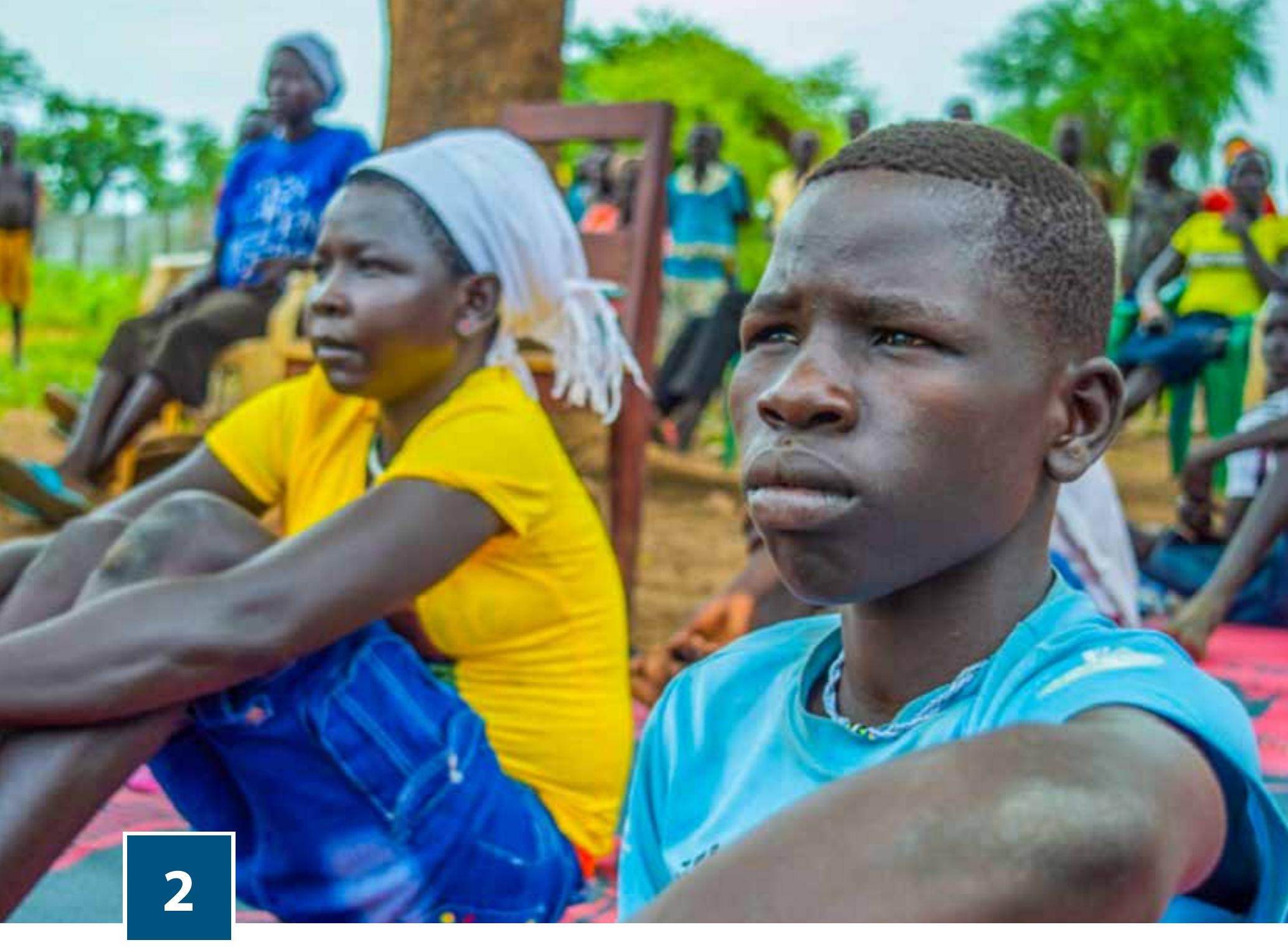

\section{COMMUNITY-BASED PROTECTION}

\section{Emerging Practice from Djibouti: Remote Community Services}

\footnotetext{
OVID-19 containment measures in Djibouti led UNHCR staff and implementing partners to begin teleworking. This had its impact on access to protection services for Persons of Concern. The current intervention emerged out of the need to maintain the continuity of protection services and engagement with refugees and asylum seekers. The intervention involves the establishment of a Helpline for refugees and asylum seekers. The Helpline is complemented by two WhatsApp groups in refugee camps - one for community leaders, and the other for community-based health workers.

The Helpline phone number is widely circulated (through information posters in Oromo, Somali, French, Arabic and English) to inform a wide range of refugees and asylum seekers (women, men, girls and boys) about its existence. The Helpline is available daily from 8 am to $5 \mathrm{pm}$, while the Whats App groups are in operation 24 hours a day. Refugee community leaders are provided with pre-paid phone credit/airtime to maintain contact with community members, UNHCR protection staff and each other.

Persons with health problems and other urgent needs, are referred by community leaders to health workers, who offer care and supportive referrals to relevant partners (national counterparts and/or UNHCR implementing partners) in accordance with the safety measures in place. Community-based health workers receive training on how to handle health issues related to COVID-19. Incoming calls to the helpline are documented along with the services provided as a result.
} 
The intervention has helped UNHCR to: maintain continuous contact with community structures; maintain case management activities; and listen to and guide refugees and asylum seekers with grievances and problems exacerbated by the pandemic.

\title{
Partner(s): $\mathrm{n} / \mathrm{a}$
}

For further information, contact:

Laura De Somer: desomer@unhcr.org

Dr. Tongna Alain Rodrigue Zoure: zoure@unhcr.org

Oubah Abdi Hassan: abdihaso@unhcr.org

\section{Case Study from Ethiopia: WhatsApp Groups for Refugee Community Leaders and Refugee Outreach Volunteers in Addis Ababa}

\begin{abstract}
A Ithough refugees in Ethiopia are located in refugee camps, diverse refugee population groups also reside in the urban setting in Addis Ababa. Some refugees move to Addis Ababa under referrals from refugee camps due to health, security, SGBV and other humanitarian reasons that cannot be addressed in the camps. Eritrean refugees constitute the largest group of urban refugees. Many reside in Addis Ababa under the Out-of-Camp Policy, a special arrangement with the Government of Ethiopia, considering the culture and historical background and ties between Ethiopia and Eritrea.

Various COVID-19 preventive measures introduced in Addis Ababa, including the closure of the reception center and limitations on face-to-face services, hindered UNHCR's communication with refugee communities residing in Addis Ababa. Addis Ababa has generally good mobile and internet coverage, and refugees in Addis Ababa enjoy good connectivity. Up to $94 \%$ of refugee households in Addis Ababa have access to a mobile phone.
\end{abstract}

As conventional communication channels with refugee communities (reception center, face-to-face meetings) became largely restricted due to COVID-19 restrictions, alternative effective channels of communication were explored. Given the solid mobile coverage in Addis Ababa, digital communication channels were established, including UNHCR WhatsApp groups with refugee community leaders and outreach volunteers.

This intervention aimed to ensure swift and effective two-way communication between UNHCR and refugee communities on a wide range of topics, including resettlement updates, messages from the UNHCR Representative to refugees, availability of services, helpline phone numbers, information on COVID-19 preventive measures, and clarification of misinformation.

UNHCR established three WhatsApp groups for refugee community leaders (15-20 members per group), representing all major nationalities in Addis Ababa, and one WhatsApp group for Refugee Outreach Volunteers. UNHCR staff (the Urban Team) serves as an administrator of all four groups, posting relevant information on the groups and replying to queries and questions.

Members of the WhatsApp groups are required to abide by certain guidelines, including not discussing cases, nor sharing sensitive or personal information about refugees.

The WhatsApp groups allow for access to relevant information for refugee leaders who are in turn tasked to cascade information to their communities, including groups with specific needs. This two-way communication is a tool to enhance empowerment and foster a horizontal partnership between refugee leaders and UNHCR.

Partner(s): n/a

For further information, contact:

Ota Hlinomaz: hlinomaz@unhcr.org

Nunu Aliyi: aliyi@unhcr.org 


\title{
Case Study from Kenya: Community Protection Committee
}

\begin{abstract}
The COVID-19 pandemic heightened the need for Persons of Concern have access to timely, relevant, and accurate information in applicable languages, while also ensuring the accessibility of communication and critical protection case management for individuals at increased risk (e.g., older persons, children, youth, Persons with Disabilities, LGBTI, and ethnic and religious minorities). The community thus proposed the formation of a committee to work alongside sectoral committees. In response to this proposal, and drawing on an AGD lens, UNHCR established a 36-member protection committee, members of which were trained on basic principles of Protection Delivery, Confidentiality, Referral Pathway and COVID-19 pandemic prevention/mitigation.
\end{abstract}

With the onset of the pandemic, camp attendance/presence by UNHCR and partners was scaled down, although work continued remotely through hotlines, bulk SMS, WhatsApp platforms and radio talk-back shows. However, refugees wanted more information from UNHCR, stating that they could not rely solely on refugee leaders to provide information.

The main component of the intervention involves leveraging existing networks of outreach volunteers and women's, children's and other groups formed and led by Persons of Concern to provide key information on critical sectoral activities and protection needs, as well as ensuring referral pathways are sustained while providing community-based protection and psychosocial support. The Committee Protection Committee provides a platform for UNHCR to harness and leverage these networks. The 36-member committee receives information from partners and conveys it the community at the block level. The committee also works with law enforcers and the camp management agency to ensure that all new arrivals are placed in the quarantine center, as well as with other sectoral refugee committees.

Other roles of the committee are to:

- Conduct door-to door/home visits to families and provide basic information on services being offered and other emerging issues.

- Safely identify the most at risk refugees in need of urgent assistance and support, and refer them to UNHCR and partners or relevant units/sectors for immediate response.

- Establish networks with community outreach volunteers, workers and structures, section and block leaders, Kenya police to facilitate referrals.

- Conduct community level information sharing on protection and prevention against contracting the coronavirus.

- Conduct regular protection monitoring by sections through section leaders to be able to gather information and inform quickly on the emerging issues such as health, SGBV, child protection, shelter, basic needs, and any other intentions.

- Provide daily updates and a report to UNHCR every Thursday of the week.

UNHCR and the Norwegian Refugee Council provide mobile phone airtime to committee members to ensure two-way communication and facilitate the provision of weekly sitreps.

Partner(s):

Norwegian Refugee Council

Refugee Affairs Secretariat

For further information, contact:

Lina Koki: koki@unhcr.org

Nicholas Midiwo: midiwo@unhcr.org 


\section{Case Study from Kenya:}

Refugee Community Workers' Use of WhatsApp Messaging Application in WASH

1 ovement restrictions during the COVID-19 pandemic led UNHCR and CARE to rely heavily on Refugee Community Workers (RCWs) to provide monitoring support for WASH activities in refugee communities. RCWs monitor activities at boreholes and within community blocks. With the pandemic came the need to use a remote platform to for the communication of monitoring information, including taking and sending photographs showing activity progress, and reporting urgent complaints such as tap leakages and pipe bursts. The WhatsApp messaging application has been employed for this purpose. The use of this application has ensured that complaints are reported and acted upon promptly, and that progress reports continue to be received and logged in at CARE WASH Office. RCWs and camp leaders have telephone contacts for WASH officers at CARE and UNHCR and can log complaints at any time during the day and at night via this application.

Partner(s):

CARE

For further information, contact:

Osman Yussuf Ahmed: ahmedos@unhcr.org

\section{Promising Practice from Rwanda: \\ Community Hygiene Work Group and the Community's Joint Effort in COVID-19 Prevention, Including Local Musicians}

\footnotetext{
The global COVID-19 pandemic and a camp scabies outbreak led to a partner coordination meeting proposal to commence a community-based hygiene workgroup in Kiziba, co-chaired by the Karongi Community-Based Protection team with support from health and WASH partners.
}

The specific aims of the practice were to: comprehensively assess the hygiene situation (knowledge, practices, and infrastructure) in the camp by developing professional inspection tools and surveys which are friendly for Persons of Concern to use; rehabilitate camp WASH facilities by sharing recommendations based on the inspection and research findings; empower community mobilizers with accountability and the ability to monitor camp hygiene and promote hygienic behavior; and mobilize the refugee community to assimilate COVID-19 prevention measures by inviting community members (especially refugee youth) to be at the center of community sensitization sessions and other evaluation activities.

The main components of the intervention are as follows:

o Community-based Inspection of hygiene: Hygiene inspection forms were developed jointly by the workgroup, and the team facilitated the hygiene inspection for households, schools, and school feeding projects. Recommendations based on the inspection results were shared with the WASH partner to improve WASH facilities before the onset of the COVID-19 pandemic in Rwanda.

- Health and Hygiene Community Worker initiative:This initiative was established in Kiziba to form groups composed of one health community worker and multiple WASH community workers for the purpose of conducting home visits and awareness campaigns.

- Community mobilization and the community's joint efforts: The Community-Based Protection team invited a refugee youth rap and music group ('DJ MC Orphans'), to facilitate a COVID-19 prevention sensitization campaign.

- Placing refugees at the center of COVID-19 research in the refugee camp: Collaborating with University of Global Health Equity, and with approval from the Ministry of Education and the Ministry in Charge of Emergency Management (MINEMA), refugee youth in Kiziba conducted a phone-based COVID-19 Knowledge, Attitudes and Practice (KAP) survey-the first of its kind carried out in a refugee camp and facilitated by refugee youth. Refugee youth were meaningfully involved in the project design and implementation, thus developing their research skills and experience. 


\section{Results}

- As part of joint COVID-19 prevention actions, the refugee community has built 122 additional hand washing stands to promote hygiene in the camp.

- Findings from the KAP survey indicated that COVID-19 related knowledge in the camp was generally high, and that the majority of the Kiziba refugees recognize community-based health communication as a significant source of information. Furthermore, Kiziba refugees scored higher in KAP related to handwashing (compared to other hygiene behaviors), partially reflecting the positive impact of the joint community effort in establishing handwashing stands.

- Women scored lower than men on COVID-19 related knowledge, due to illiteracy and limited access to phone/ radio.

Partner(s):

Africa Humanitarian Action

Alight

For further information, contact:

RuizhiZhu: zhur@unhcr.org; rwaki@unhcr.org

Speciose Buwamaria: buwamari@unhcr.org

\section{Case Study from Uganda: Community Mobilization through a Refugee Welfare Council for COVID-19 Prevention and Response}

R efuge Welfare Councils (RWCs) were established in Imvepi refugee settlement in 2017 to enhance community mobilization and coordination within the refugee population. Composed of refugee community leaders, the main role of RWCs is to identify, prevent, and respond to risks within their environment. This includes systematizing existing positive self-protection strategies; local advocacy; raising awareness of human rights, the law and medical, legal and psychosocial services; and promoting participation by different AGD groups in decisions relating to protection.

As a result of RWC efforts, protection partners and the government (Office of the Prime Minister [OPM]) and UNHCR became more receptive and responsive to protection issues and needs, while Persons of Concern became better informed of, and able to access, appropriate protection services within the settlement. With the COVID-19 pandemic, RWCs are being leveraged as a mechanism for COVID-19 prevention and response in the following areas:

- Mitigating the risks or discourage the use of negative protection strategies

- Reinforcing existing positive strategies (particularly, WHO and Ministry of Health COVID-19 guidelines)

- Supporting new, positive mechanisms of prevention and response that do not align with some preCOVID-19 conventions (e.g., staying at home, regular handwashing with soap, physical distancing, maskwearing).

RWCs are the first point of contact/reference in the settlement from whom all partners obtain information meant forPersons of Concern. From the RWCs, they obtain guidance on activity implementation during lockdown periods, and on how to identify, assess, and support those most at risk/vulnerable. RWCs thus work with both implementing and operational partners, UNHCR, other UN agencies (WFP, UNICEF, UNFPA, UN Women, etc.), and OPM on a day-today basis to ensure that quality protection services (child protection, SGBV, general protection, legal and physical protection, and community-based protection reach Persons of Concern.

With most partners teleworking during COVID-19, coordination between partners and community has been enhanced through RWCS who provide a daily status report to UNHCR, and follow up on action points fed back to them. RWCs have also helped achieve effective communication with refugee communities to mitigate resistance to new regulations sparked by COVID-19 (e.g., new food distribution guidelines and national COVID-19 prevention guidelines).

Furthermore, the leadership role of RWCs in community activities, has had positive effects. For example, RWCs have successfully fostered a communal work approach in which payment is not demanded for community work. Communities also join in to perform tasks meant for incentive workers, thus hastening any distribution efforts and minimizing large gatherings. This is evident during the distribution of food, menstrual hygiene management materials, and community events such as funerals. 
RWCs are also instrumental in liaising with the host community, independently resolving conflicts related to land, stray animals, firewood, and poles for shelter construction. They engage the host community in peaceful coexistence dialogue, only involving the OPM and UNHCR as witnesses to agreed-upon action points.

Shelter construction for Persons with Specific Needs in the settlement during the pandemic has also been carried out by RWCs who have engaged the refugee community in this endeavor. For example, in Zone 2 (Village 14), under the leadership of a female RWC, youths were mobilized to repair fiver shelters for Persons with Specific Needs during the pandemic.

Partner(s):

Danish Refugee Council

Office of the Prime Minister

UNHCR implementing partner

For further information, contact:

Willian Ngeze: ngeze@unhcr.org

Faith Kamoyo: kamoyo@unhcr.org

\section{Emerging Practice from Uganda: Community Care Coalitions}

D

ersons with Specific Needs such as Persons with Disabilities, those with serious medical conditions, women at risk, the elderly, and unaccompanied children in refugee settlements have challenges related to access to services, as well as to food, health, education, water, and shelter assistance. They also are often more susceptible to severe forms of COVID-19. With the limited number of community-based protection staff on ground as a consequence of COVID-19, a gap emerged in the identification, assessment, and referral of Persons with Specific Needs to relevant service providers. The Community Care Coalition approach, described below, aims to improve service delivery and support to Persons with Specific Needs.

With limited and diminishing resources dedicated towards the protection of Persons with Specific Needs in Adjumani operation, there was need for a more community-based approach towards addressing the needs of this population. Through a Project Partnership Agreement with Lutheran World Federation (LWF), a Community Care Coalition approach was introduced. This approach is implemented through a community-based structure called Community Care Coalition Teams (CCCTs). The latter are formed in consultation with Refugee Welfare Council leaders (refugee leaders in the different settlements). National COVID-19 restrictions have included transportation bans (except for essential services), closed borders, social distancing, ban on mass gatherings, and 30\% workplace presence. Under these circumstances, CCCTs have been instrumental in supporting Persons with Specific Needs and in ensuring that they have access to services and assistance despite the limited number of staff on ground.

\section{Main Components:}

The main components of the approach included the following:

- Preliminary institutional mapping

- Training of Community-Based Protection staff on the approach (for intervention implementation)

- Community stakeholders' meetings

- Forming a CCCT

- Action planning

- Training the CCCT

- Identifying Persons with Specific Needs in the settlements and linking them to the structure

- Training of the caregivers of Persons with Specific Needs

- $\quad$ Supporting the CCCT

- Monitoring and reporting

- Mobilizing resources to aid the implementation of community-based activities by the CCCTs 
Thus far, a total of 129 refugees ( 66 females and 63 males) are members of a CCCT. CCCT members are provided with visibility aprons for easy identification by the community. Several Persons with Specific Needs have been identified and assessed. There is increased community knowledge on community service and assistance points for Persons with Specific Needs through trainings conducted, and the referral pathway for such services/assistance has been strengthened by engaging CCCTs to act as Persons with Specific Needs guides in the settlement. The involvement of refugee leaders in inception and quarterly feedback meetings has resulted in a well-coordinated intervention. Ultimately, community capacity to respond to Persons with Specific Needs in the context of limited support has been strengthened.

Partner(s):

Lutheran World Federation

For further information, contact:

Elvira Ndanga: ndangael@unhcr.org

Elizabeth Kaboyo: Elizabeth.kaboyo@lutheranworld.org

Ajavu Patrick: patrick.ajavu@lutheranworld.org

Deogratias Acidri: acidri@unhcr.org

Prosscovia Achiro: achiro@unhcr.org

\section{Emerging Practice from Uganda: Shelter Support for Persons with Specific Needs}

\footnotetext{
Teq he temporary closure of the Kabazaana reception center due to COVID-19 led to the emergency settling of all the Persons of Concern who were residing at the center. (This was done in adherence to physical and social distancing requirements). Consequently, particularly vulnerable individuals for whom no shelters had been constructed at the time had nowhere to sleep. These individuals were unable to construct shelters on their own. This situation left Persons with Specific Needs susceptible to protection risks, as well as the risk of health issues brought about by cold and wet weather conditions.
}

As a solution to this problem, a total of 74 temporary structures have been constructed, within which 65 particularly vulnerable individuals and 9 single mothers have been housed. A community-based approach was employed in constructing the shelter, including the contribution of roofing materials (plastic sheeting provided by UNHCR as part of their Core Relief Items settling package) by particularly vulnerable individuals. Community leaders and community workers were tasked with the assessment, supervision, and follow up of the construction, while able community members contributed labor at a reduced cost, in addition to safeguarding the property of particularly vulnerable individuals. ALIGHT contributed USD 53 per shelter towards the purchase of materials such as poles, reeds, nails, transportation, and labor.

\section{Results}

The intervention has had positive results from the perspective of Persons with Specific Needs and other community members. Asked for views about the shelter support, one beneficiary responded, 'I am really happy to have a house [to] myself where I have my own privacy. Even if they tell us to go back to the reception center after this pandemic, I am not willing to go back. I am ready to settle here. Thank you, Alight.' Similarly, a community worker said, 'I appreciate the approach Alight is using where the community members are helping fellow community members in construction of these houses for the particularly vulnerable individuals, and this going to help them settle in quickly. As a community worker, I am happy to see my people being supported by Alight in this period where the pandemic has affected all of us.'

Partner(s):

Alight

For further information, contact:

Davis Mugumya: mugumya@unhcr.org

Sylvia Samanya: samanya@unhcr.org 


\section{Case Study from South Sudan: Community-Led COVID-19 Task Force}

To o prevent the spread of COVID-19 in Ajoung Thok and Pamir refugee camps, services that involved mass gathering and person-to-person contact were scaled down. UNHCR offices in the field discontinued field visits and physical community engagement. This left a gap in the usual rapid and efficient dissemination of information to the community on COVID-19 and preventive measures. The registration of refugees was also affected, with related activities carried out remotely. While awareness-raising was conducted via radio, analysis of feedback from the field showed that many refugees still had queries.

In summary, there was a need for effective communication on community activities within the COVID 19 guidelines; prompt identification and referral of Persons with Specific Needs and children at risk; and information campaigns on new remote registration modalities. An intervention was therefore developed to raise awareness on COVID-19; track COVID-19 rumors in the community; and identify and refer Persons with Specific Needs for required services during the pandemic period.

Specifically, COVID-19 taskforces were established by the refugee council leaders in the two camps, aimed at providing support to UNHCR and partners to promote two-way communication with the refugee community, and at generating information on their needs (particularly those of Persons with Specific Needs during COVID-19), and the precautionary measures being implemented. In Ajuong Thok, the Community-Led COVID-19 task force consists of 16 members (4 female and 13 male). In Pamir, the taskforce comprises 10 members ( 5 female and 5 male). Both taskforces are chaired by the camp chairperson.

Community-led COVID-19 taskforces also support UNHCR in remote registration by documenting individual information of newborn babies in their communities. They work with health partners to identify the refugees who are travelling back into the two refugee camps for monitoring by hygiene partners and immediate receipt of COVID-19 information. They also report inactive ration cards, and lost ration cards needing replacement and reactivation to UNHCR protection on behalf of refugees. The taskforces participate in the weekly Refugee Council meetings, which are also attended by UNHCR, DRC and LWF child protection. The taskforces in both camps are being supported by DRC with megaphones, face masks, refreshments, and stationery to efficiently carry out awareness-creation. sessions within the community.

\section{Partnership(s):}

Danish Refugee Council

Lutheran World Federation

For further information, contact:

Ivy Wahome: wahomei@unhcr.org

Regina Khamis Wude: wude@unhcr.org

Abraham Mijok Ayuel Dau: dau@unhcr.org

\section{Case Study from South Sudan: \\ Mask Production}

NHCR's field office in Malakal has started the production of face masks by IDP and host communities as a part of a livelihood initiative and peace-building activity to mitigate the impact of COVID-19. The Jamjang Sub-Office is looking into the possibility of engaging alumni of vocational training centers (both refugees and host community members) to produce reusable facemasks once budget availability is confirmed.

Partner(s): n/a

For further information, contact:

ViTran: tran@unhcr.org 


\section{Case Study from South Sudan: Community Radio Station}

I Jamjang refugee camps, UNHCR has worked with Jamjang FM radio to disseminate information on COVID-19 prevention. Various messaging modalities are used, including announcements and radio talk shows. Additionally, some refugees are currently developing a short drama to be aired on the FM radio station, incorporating important COVID-19 prevention and response messages. On some shows, UNHCR and implementing partner medical doctors have been engaged to assist with myth-busting. Plans are underway to air a program that will respond to questions, rumors and address some community misconceptions around COVID-19.

There is need to ensure that more Persons of Concern have access to radio sets to enhance their exposure to the messages. The preferred devices are mobile phone radios because they are multi-purpose and can serve both as mobile phones for community engagement and feedback, and as radio sets for receiving important public health information disseminated by the South Sudanese Ministry of Health as well as UNHCR and partners through Jamjang FM.

Efforts to ensure reciprocity in communication between Persons of Concern, UNHCR, and public health actors disseminating COVID-19 information through radio are underway. Ad hoc community feedback evaluations have been conducted, primarily through health staff inquiring from community members visiting health centers. The community appreciates the messages and is receiving accurate information disseminated via radio.

Partner(s):

Jamjang FM

For further information, contact:

Tamar Joanian: joanian@unhcr.org

\section{Emerging Practice from South Sudan: Humanitarian Coordination for COVID-19 Response}

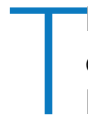
he Maban Risk Communication and Community Engagement (RCCE) Working Group has been cited on numerous occasions by the South Sudan National RCCE Technical Working Group as an example of 'a best practice of fieldlevel inter-agency collaboration.'There is no similar RCCE coordination mechanism set up by humanitarian partners in response to the COVID-19 pandemic anywhere else in South Sudan. With the onset of COVID-19 in South Sudan, humanitarian partners in Maban activated the Maban RCCE Working Group with the aim of harmonizing their approach to COVID-19 risk communication interventions. The RCCE Working Group is led by the government (Maban County Health Department) and co-led by UNHCR. A total of 21 humanitarian partners have voluntarily signed up to be members of the Maban RCCE Working Group, which meets on a bi-weekly basis.

The Maban RCCE Working Group comprises four sub-groups: 1) COVID-19 Rumor Tracking and Management SubCommittee, 2) COVID-19 Behavioral Steering Committee, 3) Monitoring, Evaluation, Accountability, Research, and Learning Unit, and 4) Mental Health and Psychosocial Support Sub-Working Group. Each group's tasks are described below.

\section{COVID-19 Rumor Tracking and Management Sub-Committee: Led by Internews and co-led by the Danish Refugee Council.}

o Training of trainers for all humanitarian partners in Maban on the effective tracking and management of rumors, misinformation, and concerns.

- Cascading of the training to implementing partner organizations.

o Development of a harmonized inter-agency online tracking tool that all partners in Maban use to report rumors.

o Periodic production of a Rumor Management Report that documents rumors circulating within the community, along with planned responses. The report is shared with the National Rumor Tracking and Management SubCommittee.

o Development of a rumor management pathway that ensures feedback on the Rumor Management Report reaches the community. 


\section{COVID-19 Behavioral Steering Committee: Led by Save the Children International and co-led by Relief International.}

o Provision of evidence-based advice to local authorities and partners on short- and long-term behavior change strategies for ensuring the uptake of COVID-19 prevention behaviors among refugees.

o Spearheading of two COVID-19 advocacy campaigns and two research projects, coupled with support for humanitarian partners to document the stories of COVID-19 change agents.

\section{Monitoring, Evaluation, Accountability, Research and Learning Unit (MEARL): Led by World Food Program and co-led by Jesuit Refugee Service and UNHCR.}

- RCCE monitoring data collection

- Humanitarian partners in Maban submit their RCCE monitoring data to the MEARL Unit on a weekly basis. This information is consolidated and submitted weekly to the National RCCE Technical Working Group. The MEARL Unit also shares exploratory data analysis reports with humanitarian partners, alongside the raw RCCE data.

- Following a Maban RCCE Working Group sensitization meeting geared toward promoting data-sharing among humanitarian partners, the MEARL Unit organized an inter-agency training for all monitoring and evaluation (M\&E) focal points in Maban County, building their capacity to use the harmonized monitoring tool.

- Supporting inter-agency RCCE research initiatives

- The MEARL Unit has supported the Behavioral Steering Committee to conduct two inter-agency RCCE research projects: 1) A rapid knowledge, attitudes and practices (KAP) survey that was conducted across the refugee camps and host community; and 2) Research on how to more effectively engage the community in COVID-19 interventions. Both research projects had the backing of the National RCCE Technical Working Group and shaped national RCCE guidance policies in South Sudan.

\section{Mental Health and Psychosocial Support (MHPSS) Sub-Working Group: Led by Jesuit Refugee Service (JRS) and co-led by the Danish Refugee Council (DRC).}

- Streamlining of MHPSS within the COVID-19 response by ensuring associated considerations are harmonized and integrated into key messaging and information-sharing among partners and community-based structures in the host and refugee communities; strengthening the MHPSS component in existing structures in the host community and refugee camps; providing support and capacity-building in MHPSS, (such as Psychological First Aid) to frontline workers, partners, and community-based structures; promoting the integration of Persons with Specific Needs into MHPSS activities; consolidating information on the situation of MHPSS well-being as well as service delivery to support gap analysis, coordinated response and advocacy for resource allocation to MHPSS partners; maintaining regular communication and connection with the national MHPSS technical working group (TWG), including participation in the national MHPSS TWG meetings; and promoting mutual learning and exchange of ideas and best practices among partners.

o The Maban MHPSS Sub-Working Group is represented on the National MHPSS TWG..

Partner(s):

Danish Refugee Council

Internews

Jesuit Refugee Service

Maban County Health Department

Relief International

Save the Children International

World Food Program

For further information, contact:

Dr. Gebrewold Petros Yohannes: petros@unhcr.org 


\section{Case Study from Sudan: Community Engagement through the Translation of COVID-19 Messages}

C ommunity teachers in Kordofan are being engaged to help translate COVID-19 prevention messages into major refugee local languages for dissemination with the help of local leaders. This initiative is meant to help create awareness and reduce further transmission of COVID-19. In some locations, such as Khartoum, communities took an active role right from the early stages of the COVID-19 response, disseminating information posters and identifying locations for them in their areas through Community Outreach Volunteers, community and religious leaders, and Sultans.

Partners: $n / a$

For further information, contact:

Annheli Aldhammar: aldhamma@unhcr.org

\section{Case Study from Sudan: \\ Community Engagement via Mobile Phone}

$\mathrm{n}$ White Nile State and other areas, both refugee and host community leaders have been selected and trained on the COVID-19 referral pathway to strengthen their capacity to respond to cases in a timely and efficient manner. They have also been supported with phone credit (airtime) to facilitate communication. In Khartoum, UNHCR is supporting a local Health Committee of Persons of Concern to work with the Ministry of Health to monitor community members for COVID-19 related symptoms. UNHCR Sudan has also launched a 'WhatsApp communication tree' mainly for Eritrean, Syrian, and Yemeni refugee communities in Khartoum. This will enable information to be distributed via WhatsApp along a network of refugee groups, facilitating timely information dissemination to and feedback from communities.

Partners: $n / a$

For further information, contact:

Annheli Aldhammar: aldhamma@unhcr.org 


\section{SGBV/RISK MITIGATION/RESPONSE}

\section{Case Study from Burundi: Mobile Phone-Based Referrals and Case Management for SGBV}

D espite movement restrictions in Burundi during the COVID-19 pandemic, UNHCR has been able to ensure continuity of care for SGBV survivors, and connection to care for newly identified survivors by issuing mobile phones and monthly airtime to key community-based protection actors within refugee camps. These actors include community leaders, security guards, host community members of the Joint Security Committee, and Sectoral Committee Chairs and Vice-Chairs. This intervention enables these actors to alert relevant implementing partners of SGBV cases and follow up on them. It also enables UNHCR to remotely transmit important messages to Sectoral Committee Chairs and Vice-Chairs, which are in turn conveyed to the wider community.

To facilitate SGBV case referral and follow-up, two facilitators per camp are appointed to liaise with social workers as needed. Urgent SGBV cases are referred to services that are available within the camp during the pandemic period. Where case management is required, the facilitators contact the social workers, the UNHCR SGBV implementing partner (International Rescue Committee), or the UNHCR SGBV focal point by phone.

Partner(s): International Rescue Committee

For further information, contact:

Gwendolyn Roeske: roeske@unhcr.org

Brigitte Mukanga Eno: eno@unhcr.org

Christine Irambona: irambona@unhcr.org 


\section{Case Study from Uganda: Training Community Structures to Identify, Report, and Follow Up on Protection Issues}

$\mathrm{D}$ uring the COVID 19 lockdown, cases requiring attention continued to emerge from refugee communities, but some leaders and community structures did not have appropriate knowledge regarding Protection and reporting processes related to this sector.

As community structures and Refugee Welfare Council leaders are situated within refugee communities, this intervention sought to draw on these community-based resources to help identify protection cases, including instances of domestic violence, and of child abuse and exploitation. In addition to identifying cases, there was a need for cases to be reported to relevant partners for intervention.

The intervention empowered these community structures by training them in the identification of protection issues and risks (SGBV, child protection, and legal), as well as in the timely reporting of such cases for intervention. This approach has helped address gaps in protection caused by COVID-19 restrictions, which limited partners' presence in the community.

Partner(s): HADS

For further information, contact:

William Ngeze: ngeze@unhcr.org

Mansur Buga: buga@unhcr.org

\section{Case Study from South Sudan: Mobile Phone Referrals and Psychosocial Counseling Sessions}

$R$ eferrals via mobile phone are being used to reach refugees in need of support, particularly in hard to reach areas withing Upper Nile, Unity, Central Equatorial and Jonglei states. In locations where mobile networks are not available, health partners and local authorities coordinate with one another, acting as emergency referral points. They then refer the cases to the International Rescue Committee (IRC) Women's Protection and Empowerment teams, and the Lutheran World Federation (LWF) for further intervention, including psychosocial counseling. IRC and LWF have provided basic training to a few refugee incentive workers in Jamjang's camps to enable them to provide basic counselling for SGBV and child protection cases in case of lockdown situations which would restrict community members' movement.

Partner(s):

International Rescue Committee

Lutheran World Federation

For further information, contact:

ViTran: tran@unhcr.org 


\section{NON-COVID-RELATED PRACTICES}

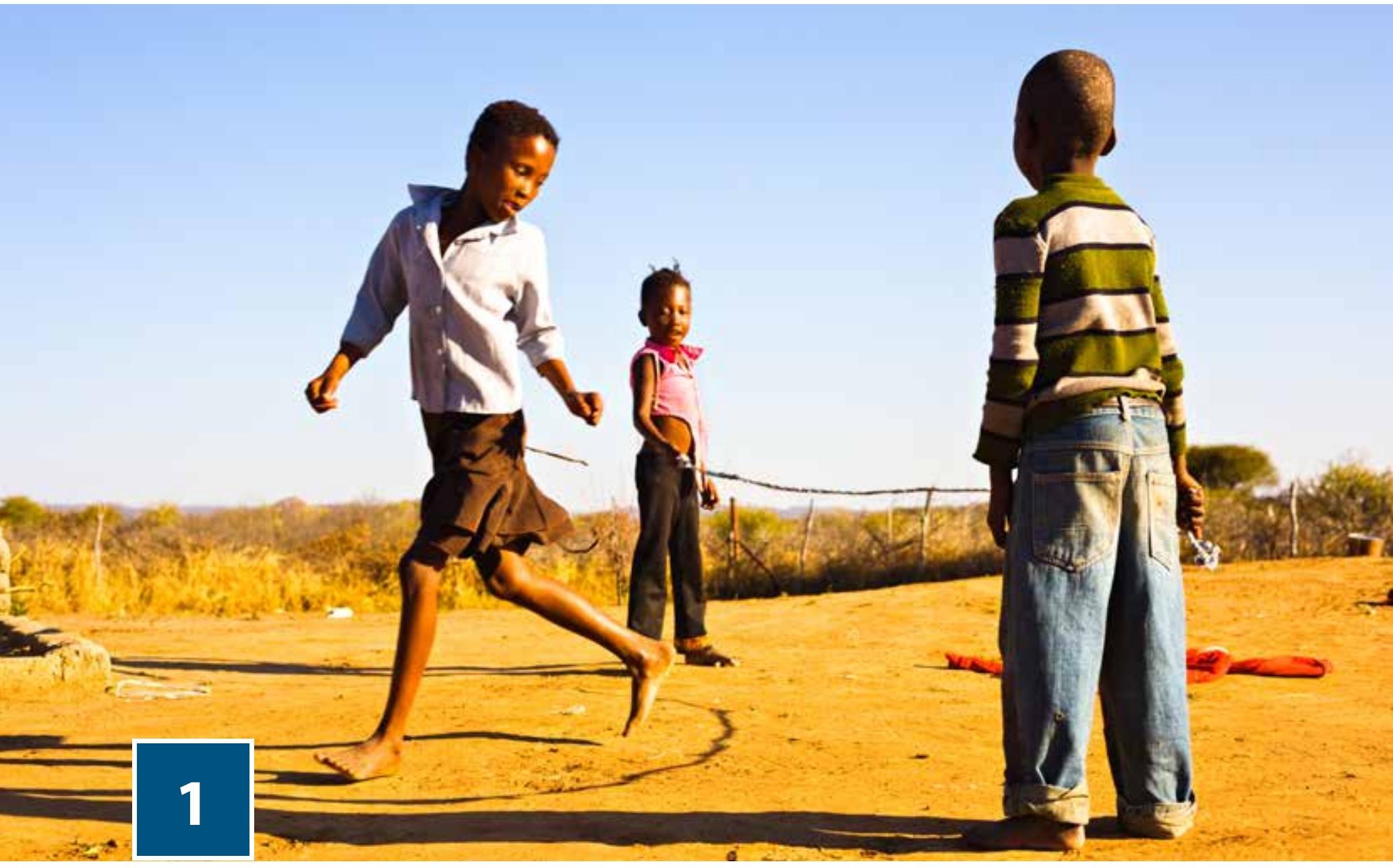

\section{CHILD PROTECTION AND YOUTH}

\section{Promising Practice from Ethiopia: Education Assessment and Programming and the Community Engagement Approach}

U NHCR Ethiopia operation's education strategy focuses on improving learning achievements, ensuring that schools are safe learning environments, improving access to secondary and higher education, ensuring that opportunities for education are lifelong and available to all according to needs, and providing access to education opportunities as early as possible during an emergency. The school enrollment rate is low due to various reasons, including economic hardship and mobility of the refugees in some camps.

In response to this reality, the operation conducts an annual Joint Assessment to assess the overall situation of Education programs, gaps and needs, and to serve as a benchmark for the planning for the subsequent year. The Joint Assessment takes place after end-year school closure between June and July, with qualitative and quantitative data collected to measure the degree of performance against the plan for the year. Questionnaires and observation checklists are also used. Participants in the Joint Assessment include the Government's Agency for Refugee and Returnee Affairs (ARRA), Development and Inter-Church Aid Commission (DICAC), Save the Children International (SCI), UNHCR, the Woreda Education Office (WEO), teachers, Parent-Teacher Associations (PTAs), community representatives, and leaders. Secondary data are drawn from the monthly and monitoring reports provided to UNHCR and ARRA zonal offices. 
During the Joint Assessment, there is a debrief of the findings at community level, fostering the participation of community representatives, and triangulation of the findings. A specific community engagement approach is employed as part of the intervention, entailing the following:

- Participation of community representatives in Joint Assessments: Religious leaders, Women Associations, Refugee Central Committees (RCCs), and other members selected by the community based on their capacity to convey the view of the community.

- Monitoring of student and teacher attendance throughout the school year by PTAs. (There is one PTA per school - 43 in total, each composed of 7 refugees, as well as Student Councils [5-8 students per Student Council in secondary schools] and Student Leaders [3-4 in each primary school]).

- Capacity-building of PTAs. For instance, by mid-2020,43 PTAs had received training on their roles and responsibilities in schools.

- The use of complaint mechanisms at schools with effective follow-up procedures (e.g., complaint boxes, focal points in schools [some of whom solely handle SGBV complaints]).

- Promoting parent participation in specially designed school activities, such as parent-led pre-school storytelling and singing sessions centered around cultural heritage. These activities take place throughout the year to enhance parent participation.

The Joint Assessment provides a platform for the community to voice its concerns, provide suggestions in relation to programs, and participate in the education planning process. Outcomes of the annual Joint Assessment are documented and used as and advocacy tool, in addition to being a reference for resource allocation and proposal development.

It is noteworthy that UNHCR has established and effective coordination mechanism with the local District's Education Office to mainstream UNHCR's education work within the national education system, enhancing sustainability, maintaining national standards, and improving acceptance. UNHCR's coordination role is to ensure an effective, multi-stakeholder response to the development needs of both the refugees and host communities. The Education Working Group, which comprises representatives such as ARRA, UNHCR, World Food Program, and other implementing partners (Save the Children International, Development and Inter-Church Aid Commission), is in place and bound by a Memorandum of Understanding. It includes 43 learning institutions (19 Early Childhood Care and Education [ECCE] centers, 21 primary schools, 3 secondary schools, and the College of Teacher Education).

\section{Results}

Since adoption of the approach in 2015, it has led to improved attendance and retention at school level. There was $83 \%$ attendance at the primary school level in 2019 and a 53\% increase in enrolment at secondary school level in refugee and host community schools combined in the first half of 2020.

\section{Partner(s):}

Agency for Refugee and Returnee Affairs

Development and Inter-Church Aid Commission

Save the Children International

Woreda Education Office

World Food Program

\section{For further information, contact:}

Muhammad Harfoush: harfoush@unhcr.org

Jael Shisanya: shisanya@unhcr.org 


\section{Emerging Practice from Kenya: Family and Community-Based Approach to Child-Headed Households Care}

K

inship or foster care is not consistently available for children in need of this service in refugee settings, owing to the diversity in cultures and practices in offering such care. Yet, many situations in refugee settings give rise to the need for foster care, including when children are left behind by parents returning to their country of origin; the abuse of some children in kinship care, necessitating short- or long-term separation; the lack of suitable foster care placements for children rescued from unsuitable care arrangements; and the arrival of unaccompanied minors in a refugee camp with no relatives identified, or suitable foster care found.

The current intervention aims to provide a reliable, community-led structure for durable care that acts as a support structure for child-headed households (CHHs); enhance the safe identification of protection concerns for children living in $\mathrm{CHHs}$ and linkage to appropriate protection interventions; and support aging-out adolescents in developing life skills and coping skills in refugee camps.

To this end, feedback meetings were held with $\mathrm{CHHs}$ to provide a forum for dialogue on issues affecting them. CHHs were asked to identify adults of their choice as mentors/supervisors whom they preferred to support them in various ways. The identified mentors were informed of their selection by a child, and brought together for sensitization sessions on parental responsibilities, and the role of mentors/supervisors under this initiative, including reporting protection concerns that the children may experience.

Follow-up feedback meetings with the $\mathrm{CHHs}$ revealed that mentors/supervisors were already proactively supporting the $\mathrm{CHHs}$ in various ways, including fetching water for the children when they were in school, cooking, and taking them to hospital when they fell sick. This proactive stance by mentors/supervisors has been commended and encouraged.

Children in need of fostering are identified via various mechanisms, such as community structures, Furaha centers (one-stop inter-agency child protection facilities), during the food distribution cycles, through reports to the Danish Refugee Council offices, or by UNHCR through bi-weekly field support or referrals from other units. Appropriate referrals are made through UNHCR's child protection unit, using the refugee camp's established inter-agency referral pathways. A child assessment is conducted through standardized best interest assessment tools and a care plan proposed in collaboration with the child. Prospects for alternate care, such as kinship and foster care, are explored first. UNHCR reviews the care plans and, neither kinship nor foster care is available, an intermediary, supervised independent living arrangement is reached between the partner and the child. To facilitate this process, the child shares names of adults that they are already comfortable with to act as mentors/supervisors, and they are sensitized to support the child as described above.

\section{Results}

Since its initiation, a total of 50 mentors/supervisors (16 male and 34 female) have been identified, sensitized, and linked with children.

\section{Partner(s):}

Danish Refugee Council

Lutheran World Federation

For further information, contact:

Amin Afridi: afridi@unhcr.org

Alexander Novikau: novikau@unhcr.org

Ina Guya: guyai@unhcr.org 


\section{Emerging Practice from Kenya: Child Protection Community-Based Structures}

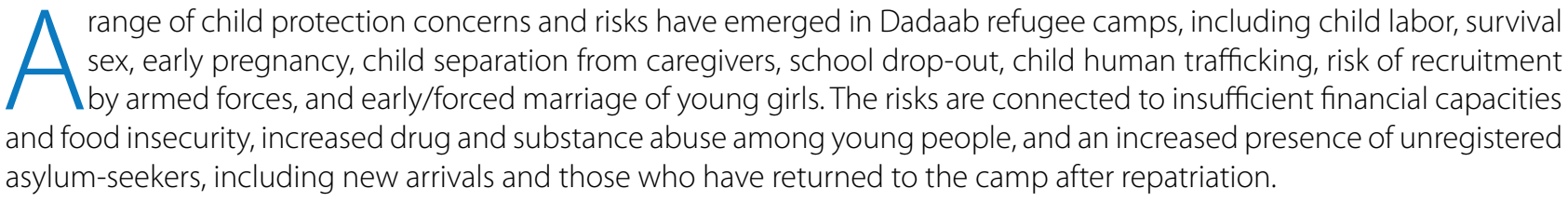

To address child protection issues in Dadaab, UNHCR works with Terre des Hommes (TdH) and Save the Children International (SCl) to implement a multi-pronged intervention, drawing on community-based structures such as Child Protection Volunteers (CPV), Community Owned Resource Persons (CORPs), Child Friendly Help Desks, Child Protection Committees (CPC), Foster Parent and Children Council, Community-Based Adolescent Committees (CBAC), Child Welfare Committees (CWC), Child Education and Welfare Center Committees and Child Mothers to empower the community to respond to existing and emerging child protection concerns. These community-based structures receive training to assist in identifying child protection concerns, and in exploring the causes and consequences. Further, they develop joint identification, prevention and response strategies related to communities' individual child protection needs and capacity.

These interventions are complemented by other approaches, including awareness-creation, mediation, information dissemination, providing referral pathways, community trainings, providing basic counselling services, adolescentled design and implementation of community actions plans, processing of low-risk cases, follow-up of referred cases, and linking the community with service providers to strengthen and promote the protection of children at family and community levels. Specifically:

- TdH provides temporary and permanent placement of children under foster care in its foster parent scheme.

- A group of refugee foster parents work with $\mathrm{SCl}$, volunteering to take care of and raise abandoned and unaccompanied minors (There are 100 foster parents in Ifo and Dagahaley and a total of 542 children under foster care).

- Community-based structures help in identifying abused children, reporting and offering referral pathways and follow-up support for such cases.

- Community-based structures such as CPC, CORPs, and CPVs are involved in low-risk child abuse case mediation processes, resulting in the reduction of low-risk cases within the communities. Along with community leaders, these same stakeholders are involved in identification and verification of beneficiaries for TdH services such as livelihood support, training and case management services.

- Child Welfare Committees help in raising awareness and sensitizing the community on the prevention of child abuse, and of harmful cultural practices, such as child marriage, FGM, and child labor. They also support SCl in enhancing child abuse victims' access to justice. They employ accessible (child-friendly, etc.) messages in their dissemination of information to children, parent/caregivers, and community members. Additionally, they pyschoeducate community members on immediate and long-term effects of child abuse, and on how to identify children in distress.

- Children assembly members hold sessions with partners during which their concerns are raised and responded to by the partners concerned. In general, community structures act as feedback reporting mechanism for beneficiaries and partners.

Partner(s):

Save the Children International

Terre des Hommes

For further information, contact:

CharityChomba: chomba@unhcr.org

Virginia Njoroge: njorogev@unhcr.org 


\title{
Emerging Practice from Kenya: Education Lessons on Community Radio
}

\begin{abstract}
n Dadaab refugee camp, many students do not have access to learning materials when schools are closed. Radio-based lessons provide an opportunity to reach a critical mass of students - particularly those who are able to learn independently, and to study with limited in-person assistance from teachers and caregivers. Radio-based lessons allow teachers to continue engaging students with relevant learning materials. When complemented with interactive applications such as WhatsApp, teachers and students are afforded the opportunity to interact more closely, where the required technology is available.
\end{abstract}

Community radio stations are critical to this intervention as many FM radio stations that broadcast national school lessons are inaccessible by refugee camps. Community radio stations therefore provide refugee students with equal access to the learning materials provided to national students.

The intervention entails the use of a radio schedule, which is circulated via bulk SMS and through community leaders a week in advance of the education broadcast. Radio announcements are also used to inform refugees and the host community about the planned radio program line up. Students are required to tune into the radio station according to the pre-communicated schedule. Families sharing a common household fence can share the radio device by placing it near the fence and increasing the volume.

Radio scripts for each topic are prepared by select subject teachers and printed out. One teacher involved in preparing the scripts is selected to rehearse a day in advance before presenting the lesson over the radio. WhatsApp groups composed of students and teachers are used to foster direct interaction.

\section{Results}

Plans are underway to acquire ready-to-air education content from the Kenya Institute of Curriculum Development. This national education content will replace the current radio scripts, once available, while teachers will continue to maintain contact learners through the WhatsApp tree groups.

\section{Partner(s):}

For further information, contact:

Clara van Praag: vanpraag@unhcr.org

Alan Mwika: mwika@unhcr.org

\section{Case Study from Kenya: Engaging School WASH Club Members}

\footnotetext{
The Norwegian Refugee Council's (NRC's) Water, Sanitation, and Hygiene (WASH) intervention involves the inclusion of children and youth and a multi-faceted and multi-level approach. In schools, NRC works closely with school WASH club members (20 members per school), training them on hygiene and sanitation approaches. In turn, trained WASH club members reach out to school mates as peer educators, passing on the information and approaches they have learned. NRC also trains teenage girls in schools on menstrual hygiene management.
}

At the community level, the NRC WASH team works with youth to strengthen the participation of community-based organizations and self-help groups in WASH activities. Given the focus of NRC's approach on children and youth, NRC staff are trained on the Child Safeguarding Policy, and NRC national staff sign onto this policy as part of their job descriptions.

\author{
Partner(s): \\ Norwegian Refugee Council
}

For further information, contact:

Moe Miyahara: miyahara@unhcr.org

Oscar Nabiswa: nabiswa@unhcr.org

John Wagacha Burton: burtonj@unhcr.org 


\section{Emerging Practice from Uganda: Community Connectivity Project}

T

he Community Connectivity project in Imvepi settlement is a youth-managed initiative which responds to the issue of youth idleness in the settlement, while attending to phone-charging needs in the community. A stable and constant power source for phone charging is a key challenge in the settlement - one that hinders communication and coordination between UNHCR, its partners, and the community, including Refugee Welfare Councils (RWCs). The project began after the solar-powered community phone charging booth was installed. The intervention entails connecting community buildings (with a primary focus on Protection Desks and Information and Support Centers), by enabling WiFi access and by transforming the buildings into connected centers. With this connectivity in place, they become places where refugees can access information, communicate with UNHCR and partners through the newly established helpline (as part of the Feedback Referral and Resolution Mechanism [FRRM]), or remotely contact loved ones using variety of messaging/voice call applications.

At the onset of the project, youth actively mobilized and participated in community awareness-raising on the phonecharging activity at the center, reaching out to all Zone 1 villages, including worship places, market, general food distribution locations, and sports events. As part of this process, youth reached agreements with the community on charging rates for various devices: Ugx.300/- for ordinary phones, Ugx.400/- for smart phones, Ugx.500/- for torches, and Ugx.700/- for laptops. Young people also involved the RWCs in community awareness sessions to build a strong consensus over the project. The RWC II Chairperson in particular has provided the youth with very strong support on this project in terms of advocacy.

On commencement, the project only had the capacity to charge a few devices at a time. However, UNHCR recently supported the center by providing it with 20 phone chargers. Currently, it has the capacity to charge 12 to 15 phones and other appliances (laptops, torches, and power banks) daily.

The availability of the connectivity has enhanced peaceful co-existence, bringing together both refugee and host community youth at the center to play board games together and interact in other ways. Thanks to this platform for interaction, the youth are planning a meeting with their host community peers at the center to incorporate these peers into the connectivity project team.

Young people have also initiated the sale of mobile phone airtime from the savings of the phone-charging initiative. This is a convenience appreciated by many Persons of Concern who no longer have to travel lengthy distances for this purpose. The youth also advocated for a 2-stance latrine at the center. This was eventually provided by the International Rescue Committee, with young people making a contribution by excavating the pit latrine.

UNHCR and the Office of the Prime Minister have overall responsibility for the project, while the protection partner and RWCs are responsible for day-to-day monitoring of the project results.

Partner(s):

Danish Refugee Council

Office of the Prime Minister

For further information, contact:

William Ngeze: ngeze@unhcr.org

Faith Kamoyo: kamoyo@unhcr.org 


\section{Emerging Practice from Uganda: Access to Secondary Education}

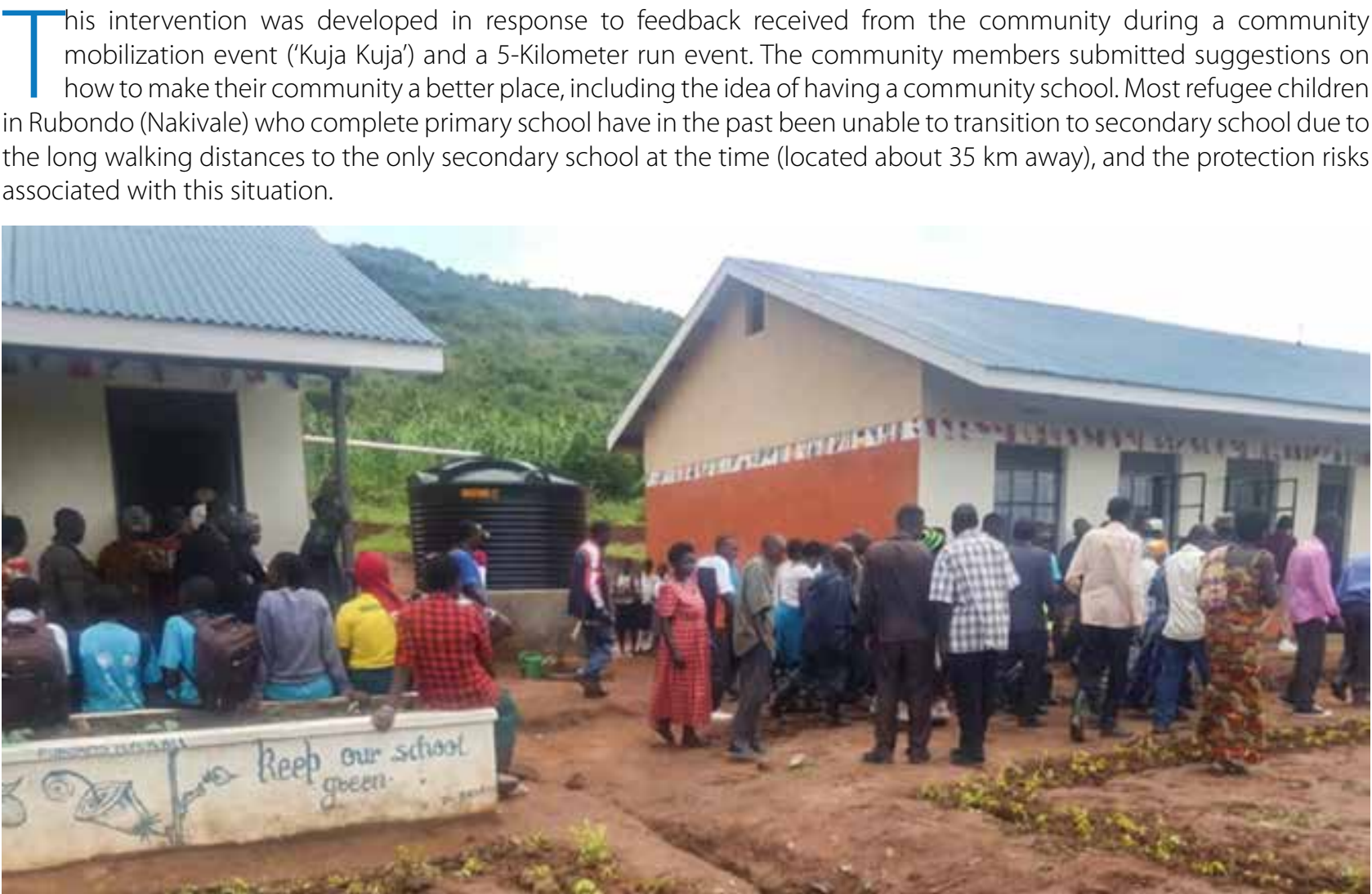

The intervention effort is led by community members, who mobilize all parents in the refugee and host community, encouraging them to send their children to school. Community members also continue to engage in monthly community participation activities, including cleaning the school compound and demarcating and protecting school boundaries from encroachment. Parents contribute school tuition of Ugx 100,000 (about USD 27) per term to facilitate the running of the school, including payment of teacher salaries.

\section{Results}

- Enrollment increased from 76 students (32 female) at the end of 2019 to 110 (52 female) in March 2020, prior to the closure of the schools due to COVID -19 pandemic.

- Retention was high, with 93\% of the students that enrolled in 2019 retained by the beginning of 2020.

- The school has a school feeding program through which meals are provided, which also facilitates retention.

- Community positivity towards the school is encouraging. The community supports this community led initiative, by paying school tuition payments and providing other requirements for their children to access education at the school, such as scholastic materials and school uniforms.

- Coordination and collaboration with other actors is robust The school management works very closely with the District Local Government, UNHCR, Office of the Prime Minister, Windle International Uganda (the UNHCR education partner) and Alight, the partner supporting its development.

- Infrastructural development: With support from stakeholders, notably, Alight, a science laboratory has been constructed and completed. This is a Ministry of Education and Sports requirement for all secondary schools for delivery of science lessons.

\section{Partner(s):}

Alight

District Local Government

Office of the Prime Minister

Windle International Uganda

\section{For further information, contact:}

Davis Mugumya: mugumya@unhcr.org

Sylvia Samanya: samanya@unhcr.org 


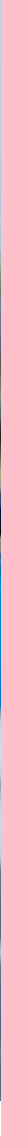

\section{COMMUNITY-BASED PROTECTION}

\section{Emerging Practice from Djibouti: Communities' Contribution to TB Case Finding among Refugees}

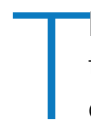
his practice aims to improve Tuberculosis (TB) case finding in refugee settings and to promote early initiation on treatment through community involvement activities. It also seeks to enhance support to TB clients by providing care closer to home. The intervention consists of six main components, namely, the formation of health committees, capacity-building of community health workers (CHWs) and healthcare workers (HCWs), quarterly review meetings, advocacy meetings, nutritional support, and treatment close to the community. These components are elaborated upon below.

- Establishment of a 10-member health committee in each camp to serve as an interface between the health system and to ensure the communities are actively involved.

- Training of $17 \mathrm{CHWs}$ to implement house-to-house community screening of TB, couple with training of $15 \mathrm{HCWs}$ on TB and Multi-Drug Resistant TB management.

- Quarterly review of the community case finding results by the trained HCWs. The results are discussed with the wider community via a meeting led by community block/section leaders, the regional TB representative, and the UNHCR focal point. Meeting participants agree on recommendations for improving TB management in their communities.

- Advocacy meetings with community leaders to help fight stigma against TB and HIV and patients.

- Nutritional support is provided by UNHCR to the identified cases. The national treatment program now permits TB patients to receive treatment at camp level, thus benefiting from the support of their communities.

\section{Results}

With the support of community health committees and leaders, CHWs have thus far referred a total of 140 suspected cases to health centers for diagnosis. This process led to the identification of 12 actual TB cases by mid-year - i.e., twice the expected proportion (of 6 cases) by the mid-year period.

\section{Partner(s):}

Intergovernmental Authority on Development

Djibouti Ministry of Health

For further information, contact:

Dr. Tongna Alain Rodrigue Zoure: zoure@unhcr.org 


\section{Emerging Practice from Djibouti: Community Participatory Solid Waste Collection by Persons of Concern}

P reviously attended to by a third party, the collection and removal of solid waste from refugee households was eventually handed over to Persons of Concern due to budget reductions. During the initial stages, waste collection awareness campaigns had sparse community participation. UNHCR implementing partner, Direction de l'Hydraulique Rurale (DHR), therefore proposed adopting similar collections strategies as those used in Djibouti cities.

Refugee community leader co-developed the household waste collection strategy along with DHR, identifying various waste collection points and ensuring that no household was overlooked. This collaboration led to the establishment of a weekly waste collection schedule, a transit route, and collection points, all of which were communicated to community members. Two motorcycles were purchased, to ply the transit route daily, while refugees wait at the various collection points in their areas with household waste for disposal.

To ensure the quality of household waste collection and management services, DHR recruited refugees as hygiene promoters. The latter benefit from capacity-building sessions to ensure the maintenance of a healthy living environment in their respective communities.

The hygiene maintenance that this intervention promotes can also serve to prevent neighborhood conflicts between the host community and Persons of Concern. Furthermore, as refugee community leaders are involved from the development of the strategy to its implementation, and also oversee the activity, they are at liberty to review and adjust the intervention as needed.

\section{Partner(s):}

Direction de l'Hydraulique Rurale

Office National d'Assistance aux Réfugiés et Sinistrés

For further information, contact:

Moustapha Hassan Ahmed: mhaktulli@gmail.com

Abdo Ali Kayad: abkalay@yahoo.fr

\section{Emerging Practice from Ethiopia: \\ Organization of an Election of Refugee Representatives in Line with Age, Gender, and Diversity (AGD) Considerations}

- ollowing security concerns arising from ethnic clashes that claimed refugee lives in the Kule refugee camp, Agency
for Refugee and Returnee Affairs (ARRA), the camp management agency, suspended the Refugee Central Committee
(RCC) (which was suspected to have been compromised) and temporarily established a Peace Committee that had
the dual responsibility of conflict resolution and community leadership. There was a need to establish a new elected and
trusted refugee representative committee to take over from the Peace Committee and manage the Kule refugee camp
community.

The two-pronged intervention involved developing election guidelines that provided a common understanding among all stakeholders and promoted fairness, transparency, and accountability in the electoral process; and facilitating the organization of the election of refugee representatives drawing on the AGD approach.

While UNHCR and ARRA facilitated the development of the election guidelines and the organization of the election, Peace Committee members (acting RCC members) provided their inputs on the document, and led the entire election process by briefing the wider community on the guidelines, mobilizing the community for the election, and supervising the election in each sub-clan as an electoral committee. The involvement of the Peace Committee (composed of all subclans) from the development of the election guidelines to the actual election, aimed to prevent any protection concerns resulting from the establishment of a new RCC. The election guidelines contained provisions for the inclusion and protects all categories of persons, including Persons with Specific Needs. 


\section{Results}

As a result of this emerging practice, a new Refugee Central Committee has been successfully established, comprising 15 elected members. For the first time, all clans and persons with specific needs are represented in the leadership structure. The number of women within this leadership structure also increased from 1 to 5.

Partner(s):

Agency for Refugee and Returnee Affairs

Refugee Peace Committee

For further information, contact:

Dismas Nyamwana: nyamwana@unhcr.org

Mekdes Aschalew: aschalew@unhcr.org

\section{Emerging Practice from Ethiopia: Community Empowerment Guidelines}

\footnotetext{
ommunity Empowerment Guidelines were collaboratively developed by Eritrean refugee community leaders and UNHCR, Agency for Refugee and Returnee Affairs (ARRA), and other non-governmental organizations (NGOs) with the objective of providing financial support and assets that would empower refugees to support themselves, and empowering the community by building its capacity to design and manage protection activities in refugee camps.
}

To this end, the Rehabilitation and Development organization (RADO), a UNHCR implementing partner working with Persons with Disabilities and older persons received a budget of ETB 770000 (USD 24,062.5) to support its associations in establishing self-help groups through Community Empowerment Projects in all four Tigray camps: Adi Harush, Mai Aini, Hitstats, and Shimelba. RADO oversees 8 associations (two per camp) - 4 centered on Persons with Disabilities and 4 focused on older persons). The Community Empowerment Guidelines provided a framework for Community Empowerment Projects, and the activities for doing so included:

- Sensitizing the beneficiaries (in this case, RADO associations) to guidelines during a meeting with each association, with UNHCR providing guidance when needed. Questions and answer sessions were included to ensure that refugees fully understood the guidelines, and the latter were translated into Tigriana and shared with refugees.

- Drafting of each association's project proposal by refugees in consultation with their community members, using a community empowerment form. The main components of proposal included needs, objectives, and management plans of the project.

- Providing additional support for proposal development to associations in need of it. This ensured the timely submission of proposals and also included coaching on budget and activity planning.

- The identification of project sites/spaces in coordination with camp managers.

- Review sessions with each association to assure the quality of proposals before presentation to a selections committee.

- Training of the management team by RADO on small business management before the disbursement of cash and assets.

- Monitoring by a joint team (UNHCR, Refugee Central Committee, RADO) through periodic project site visits.

- Issuing a monthly allowance to those directly managing the project.

\section{Partner(s):}

Agency for Refugee and Returnee Affairs

Rehabilitation and Development Organization

For further information, contact:

Yamah Massaley: massale@unhcr.org 


\section{Case Study from Kenya: Sports for Protection and Prevention of Violence}

S ports has played an important role to promote the protection of women, men, girls and boys in different sectors such as education, health and peaceful coexistence in Kakuma refugee camp and Kalobeyei settlement. Paradoxically, sports in Kenya (football, in particular) may also trigger strong emotions and conflict among groups that share similar ethnic and cultural identities.

In an effort to curb violence and promote peaceful co-existence among refugees and host communities, UNHCR and partners established a new model for sports involving multi-national, multi-ethnic teams geared toward fostering a stronger bond between the various people groups represented by refugee and host community members.

The new model promotes behavior change among girls, boys, and young men and women, along with 'Do No Harm' principles through a number of key activities:

- The creation of male ('Kakuma Premier') and female ('Diva League') football leagues: The leagues have a requirement that participating teams be multi-ethnic and multi-national.

- Professional Training of Referees: UNHCR and partners provided professional trainings to referees, including host community members, as historically, violence has been triggered by perceived erroneous decisions of referees.

- Professional Training of Coaches: As respected heads of football teams who play an important role in accepting decisions (even when perceived to be erroneous), and calming players and supporters who may have little understanding of referees' calls, coaches also receive training under this intervention.

- Management of Sport Fields and Teams: Sports Council are bodies elected by players to run sports as stipulated by the Kakuma Premier League. Sports Councils and Secretariats have been created in each zone for this purpose. They play an important role in planning sports activities to public satisfaction in order to avert potential conflicts. Sports Councils members are trained on leadership skills, resource allocation, and management.

- The creation of 'Kakuma United Football Club': To strengthen peaceful co-existence and promote interest beyond parochial stakes, UNHCR and partners have advocated for the creation of this club that brings the best players from the Premier League teams to compete at the Kenya National Football League Second Division. Kakuma United Football Club players have attained local celebrity status, becoming increasingly known by refugees and Kenyans alike.

- Mentorship Program: Mentorship trainings are held, targeting girls or young women, and boys in Kakuma. Through guided meetings with well-known and respected players from Kakuma Divas (Women's) League, girls and women are sensitized and equipped with knowledge and skill on various female protection issues. Training sessions have been offered virtually during the COVID-19 period, with enrollment by several players.

- Kalobeyei Sports Complex: The Complex consists of two main venues, i.e., the Stadium and the Multipurpose Center (Hall). It aims to create a safe and supportive environment for refugees and the host community. Activities within this space will also support young people to become active protection actors and agents for positive change, ensuring the wider participation of women, girls, refugees with disabilities and other marginalized groups.

Partner(s):

Lutheran World Federation

For further information, contact:

Amin Afridi: afridi@unhcr.org

Alexander Novikau: novikau@unhcr.org

Astone Ananda: ananda@unhcr.org 


\section{Emerging Practice from Kenya: Community-Based Care Arrangements for Unaccompanied Adults (Including the Elderly) Living with Severe Forms of Disability and Mental Health Illness}

\footnotetext{
T his intervention aims to: identify and assess unaccompanied adults living with severe forms of disability and mental health illness (including the elderly) in refugee settings; provide community-based care arrangement for such adults; ensure they receive appropriate protection interventions and assistance; and reduce stigma against such adults. The intervention was piloted for the target population who happened to be long-stayers at reception centers due to their specific needs. Previously, this population could not be relocated to the community as they required additional community support.
}

This target population is identified by the community, partners, or UNHCR staff, and referred to a specialized services team within UNHCR protection using Kakuma refugee camp's established inter-agency referral pathway. The specialized services team screens all referrals and assigns them for case management to the relevant partner, drawing on an appropriate multiagency approach. The partner carries out an assessment in consultation with the person of concern, his/her family and relevant members of the community. As part of the case plan, the partner identifies a family that would be willing and able to support the vulnerable adult, or broader community-support via a buddy system (generally consisting of neighbors, friends, partner refugee staff, youth leaders, and community leaders who are willing and able to provide support). Within the buddy system, support functions are identified and assigned, including, for example, support with cooking, laundry, or collecting rations.

The process of placing a vulnerable adult in family-based care, involves a series of consultations by the partner coupled with home visits to ensure that the care and placement modalities are well understood and agreed to by the vulnerable adult and the support family. UNHCR and partners provide further needed support, including relocation, shelter, core relief items, re-assignment of assistance location, and designation of alternative care arrangements. Following placement, the partner conducts regular monitoring visits, builds the capacity of the community involved, and ensures that the vulnerable adult and the family are informed on how to contact UNHCR and/or the partner should any issues arise.

In summary, the steps taken during this intervention are as follows:

- Target group cases are identified.

- Agency caseworkers ensure the person of concern is registered, and then referred to relevant agencies for an appropriate multifunctional approach.

- An assessment is conducted, including a vulnerability assessment. The person of concern consents to meet the identified persons considered.

- A pre-screening exercise is carried out, including meeting with the identified person, determining their willingness to participate in the program, conducting a home visit, and assessing accessibility and the general home environment. If the initial meeting was not held at home, the modalities of placement are explained.

- Processing takes place, which includes determining any required relocation logistical arrangements, e.g., shelter, non-food items, ration collection, firewood, etc.

- Relocation and placement at home occurs by holding a meeting with the concerned individual and the caregiver with his/her family, if available, and listening to any concerns/questions.

- Follow-up and monitoring are undertaken, including conducting bi-weekly monitoring for the initial two months at the home, addressing any concerns that may arise, conducting monthly monitoring for another three months and thereafter, where applicable, closing the case.

\section{Results}

In the first month of this community-based care arrangement, a total of 9 unaccompanied adults living with severe disability or mental health illness, including the elderly, were identified and linked to support offered under the program.

Partner(s):

African Inland Church

Humanity and Inclusion

International Rescue Committee

Jesuit Refugee Service

For further information, contact:

Leana Podeszfa: podeszfa@unhcr.org

Alexander Novikau: novikau@unhcr.org

Caroline Jeptoo: jeptoo@unhcr.org

Astone Ananda: ananda@unhcr.org 


\section{Case Study from Kenya: Avoiding Water Contamination with Lid Strings for Jerrycan Covers}

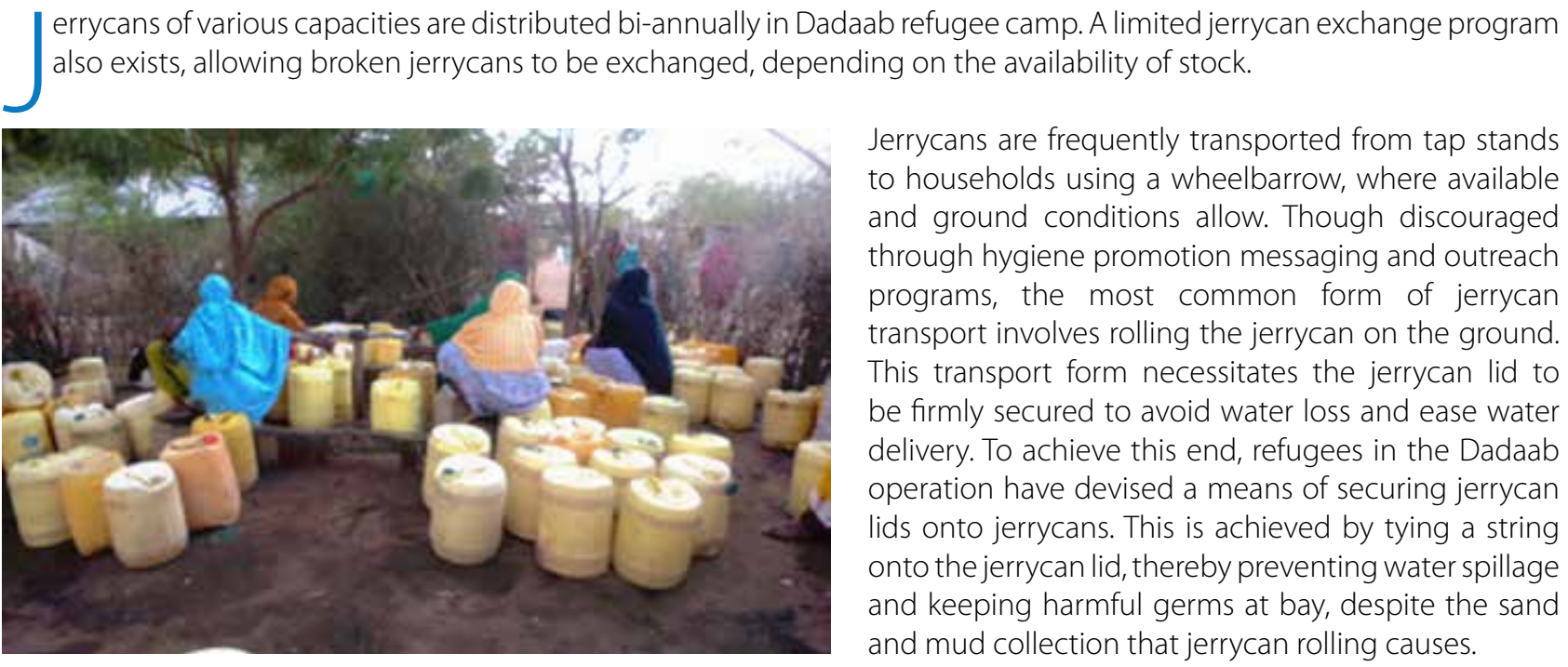

Partner(s): n/a

For further information, contact:

Osman Yussuf Ahmed: ahmedos@unhcr.org

\section{Case Study from Kenya: Market Water Committees}

- nsuring an increased or maintained supply of potable water in refugee contexts remains an important goal. Market
Water Committees (MWCs) were established in Dadaab refugee camps to distinguish domestic water from 'business
refugees and the host community business sector in the three camps. The long-term aim of the initiative is to transition the
management of water services for refugees to a water utility company managed by various stakeholder representatives,
similar to urban water systems approaches in Kenya. UNHCR and CARE have commenced a pilot 'Water ATM kiosk' for a
MWC. If successful, the Water ATM kiosk will be a precursor to monthly bulk water allocation for a family size of about 10
people. Such a family size would be allocated 20 liters of water per capita daily, translating to 6,000 liters per month. The
allocated amount of water will be redeemed from the Water ATM kiosk at any time of the day. Water use exceeding this
level have to be paid for.

Partner(s):

CARE

For further information, contact:

Osman Yussuf Ahmed: ahmedos@unhcr.org 


\section{Emerging Practice from Kenya: ‘The Spiritual Connection' - Refugee-Led Urban Churches as Resources}

$\mathrm{P}$ ersons of Concern communities in Kenya's urban areas require coping mechanisms to enable them survive the challenges of city life in a foreign country. Faith can contribute significantly to promoting social protection and conflict resolution while helping to maintain a sense of identity among refugees.

In Nairobi, some refugees have set up their own places of worship. These centers of worship are primarily designed to meet refugees' spiritual needs, while replicating the church experience from their country of origin. One such church in the outskirts of Nairobi (Kitengela neighborhood) is led by a refugee pastor, who also happens to be a refugee community leader. The church brings together a congregation of refugees who gain access to a range of services, including temporary accommodation for new arrivals in the area; introduction of new arrivals to other refugees and networks; food and material support (despite limited resources); and basic language training, which enhances communication and livelihood.

The church is also a member of the 'Kitengela Pastors Fellowship', a forum which provides networking opportunities with host community churches, and through which some of the conflicts between the refugees and their hosts have been resolved.

\section{Results}

Refugee churches provide a sense of belonging to refugees, which is important for psychosocial wellbeing. The linkage between refugee churches and host community churches opens up opportunities for dialogue and support for Persons of Concern. It also provides practical solutions to some challenges refugees face in relating with the local administration and police. The interaction between the refugee church and host community churches has also enhanced refugees'access to information and useful contacts for support during the COVID-19 pandemic.

\section{Partner(s):}

Commmunity-Based Protection Working Group

Refugee Affairs Secretariat

For further information, contact:

Jackson Karugu: karugu@unhcr.org

\section{Case Study from Kenya: Community WASH Committees}

Kin akuma refugee camp and Kalobeyei settlements are characterized by semi-arid terrain, extreme heat conditions, river flooding, and general water scarcity. To foster community ownership of WASH-related issues, each community block in Kakuma sets up a WASH Committee.

WASH Committees are voluntary groups composed of four to six persons who are responsible for overseeing any issues that emerge in the WASH sector. Besides two block leaders who are automatically members of a block's WASH Committee, the rest of the membership is determined through discussion with community members. Usually, the selected members represent different categories of community members, such as women, youth, minorities, persons with specific needs, and persons living with disabilities.

In Kakuma and Kalobeyei, water is supplied via 19 boreholes through a network of elevated steel tanks and a distribution network reaching tap stands in the community. The boreholes are powered largely by generators, but also by solar panels, where available.

Crowding and long waiting times at water points often result in stress and frustration for water-fetchers. Conflicts at water points can lead to community tension and disengagement. To mitigate this, WASH Committees develop distribution schedules through dialogue with the community. Each family is then specifically assigned to one of three rounds of water distribution. Water is distributed three times in a day (once in the morning, noon, and evening before dark). Distributing water before dark ensures the safety of women and girls, whose task it often is to fetch water. 
Select community members are trained in the repair of WASH facilities, including tap stands, which often break down. Involving the community in engaging and resolving their own WASH-related issues, fosters community empowerment and capacity-strengthening.

The Norwegian Refugee Council (NRC) dedicates protection national staff to support WASH Committees, and to ensure that WASH projects are implemented according to protection standards, principles and guidelines, with an emphasis on conflict resolution and program safeguarding. NRC's WASH sector works closely with UNHCR departments (protection, community unit, field unit, health, education) to address any WASH-related protection issues. Special inclusion and emphasis is placed on women-headed households, unaccompanied children, the elderly, and Persons with Specific Needs. NRC involves the community during all phases of the WASH project cycle management. NRC WASH team participates in a monthly community leaders and partners meeting to provide feedback and share information with the community. NRC also has a complaint, response, and feedback mechanism which encourages community dialogue.

\section{Partner(s):}

Norwegian Refugee Council

For further information, contact:

Oscar Nabiswa: nabiswa@unhcr.org

Moe Miyahara: miyahara@unhcr.org

\section{Case Study from Rwanda: Village Governors}

1 istorically in Mahama camp, UNHCR reports were only sparsely informed by refugee accounts, relying primarily on partner reports and occasional monitoring with limited staffing. The need to involve refugees more deliberately became apparent during the onset of the emergency in the camp, when it was discovered that refugees at the grassroots level lacked information on how to solve their own problems and where to access services in Mahama camp. UNHCR's leadership designed the'Village Governors'intervention to: ensure Persons of Concern access accurate information in a timely manner; strengthen refugees' capacity to independently resolve minor issues within the community without intervention by implementing partners; and monitor service delivery by implementing partners via community accounts, holding partners accountable where discrepancies occur.

The Village Governors initiative involves dedicating one UNHCR staff member to oversee one village in Mahama refugee camp, which accommodates approximately 250-480 families. Such staff members are tasked to ensure that refugees residing in their villages are adequately informed about services provided in the camp, receive a range of available services, and are able to solve minor community issues (or to put mechanisms in place to do so ).

Nicknamed 'Governors,' these UNHCR staff hold a variety of meetings with village leaders, collaborating with them to convene mass gatherings once a month or on a quarterly basis. These community-wide sessions are used to disseminate information about available services, camp rules, safety and security, durable solutions, child protection and SGBV, and community mobilization messages for generating solutions to community problems independently. Question and answer sessions are built into these meetings.

Governors sometimes invite government officers and different implementing partners to convey important messages or to provide information on challenges raised by the community. Governors also participate in community work alongside the residents of their villages prior to meetings as a way of encouraging community work and solidarity. Occasionally, governors hold ad hoc meetings when new forms of assistance are available, or an urgent message needs to be shared with residents (e.g., the roll-out of cash-based interventions, gas distribution, verification exercise information).

Reports from these meetings are shared with the UNHCR management team, which helps similar patterns to be identified, critical lessons to be gleaned, and interventions to be provided as needed.

Partner(s): n/a

For further information, contact:

Emilia Lobti: lobti@unhcr.org; rwaki@unhcr.org 


\section{Emerging Practice from Rwanda: Protection Mobile Clinics}

W

leekly coordination meetings between UNHCR and implementing partners in Mahama refugee camp (for the purpose of coordinating all protection activities) revealed the need to spend more time in the refugee community, ensuring that Persons of Concern were receiving the right messages, understood the referral pathway for child protection and SGBV cases, and had an opportunity to ask questions related to any protection issues in the camp. Working jointly to this end was decided upon as the best strategy for providing a strong front and response to issues in a concurrent manner. A rotation schedule was therefore developed by UNHCR for partners' work in coordinating and organizing such joint community visits in close collaboration with village leaders in the first three weeks of each month. The last week of the month is reserved for planning for the Protection coordination meeting, and ensuring partner and community leadership participation.

As a result of these efforts, every Thursday, a mass village meeting is organized in the community, with the venue rotating among all 18 villages of Mahama camp, in addition to schools, market places, and the host community. The meetings are jointly organized by UNHCR, the Ministry in Charge of Emergency Management (MINEMA), and partners in charge of Protection, in collaboration with village and community leaders, Social Affairs and community-based structures (anti-SGBV groups, youth groups, community activists, drama troupes, dance groups). This practice is referred to as the 'Protection Mobile Clinic.'

The Protection Mobile Clinic typically commences with an introduction of the village/community leader, followed by a dance, song, poem, or drama skit on a given protection issue in the community. Partners then give presentations on available services and responses to/prevention measures for crime, child protection, and SGBV issues.

Protection Mobile Clinics offer a platform for UNHCR, MINEMA and partners to sensitize the community on various protection topics and disseminate the most up-to-date information and announcements. A session for questions, complaints, and feedback from the community is incorporated into the intervention, along with responses from partners.

Individual and sensitive cases are never addressed during the clinic visits. Instead, partners provide general information on where to reach different institutions if needed, and self-identified cases that emerge during the sessions are referred to relevant partners. The community is continuously reminded about their rights, obligations, and respect for the camp rules and regulations.

The mobile clinic is conducted in an engaging and enjoyable way, with partners community leaders employing respectful and empowering language. Child-friendly language is used while addressing children, and the participation of women, men, girls and boys from all backgrounds is encouraged through mobilization.

\section{Results}

Protection Mobile Clinics are a means of proactively reaching out the community, rather than expecting community members to seek out partners. Through this practice, partners are able to remain in touch with the realities that refugee communities face, and receive various complaints and feedback, along with an opportunity to respond to them.

\section{Partner(s):}

Ministry in Charge of Emergency Management

Save the Children International

Alight

Adventist Development and Relief Agency

International Committee of the Red Cross

Legal Aid Forum

Prison Fellowship Rwanda

For further information, contact:

Emilia Lobti: lobti@unhcr.org 


\section{Promising Practice from Rwanda: Voluntary Savings and Loan Associations}

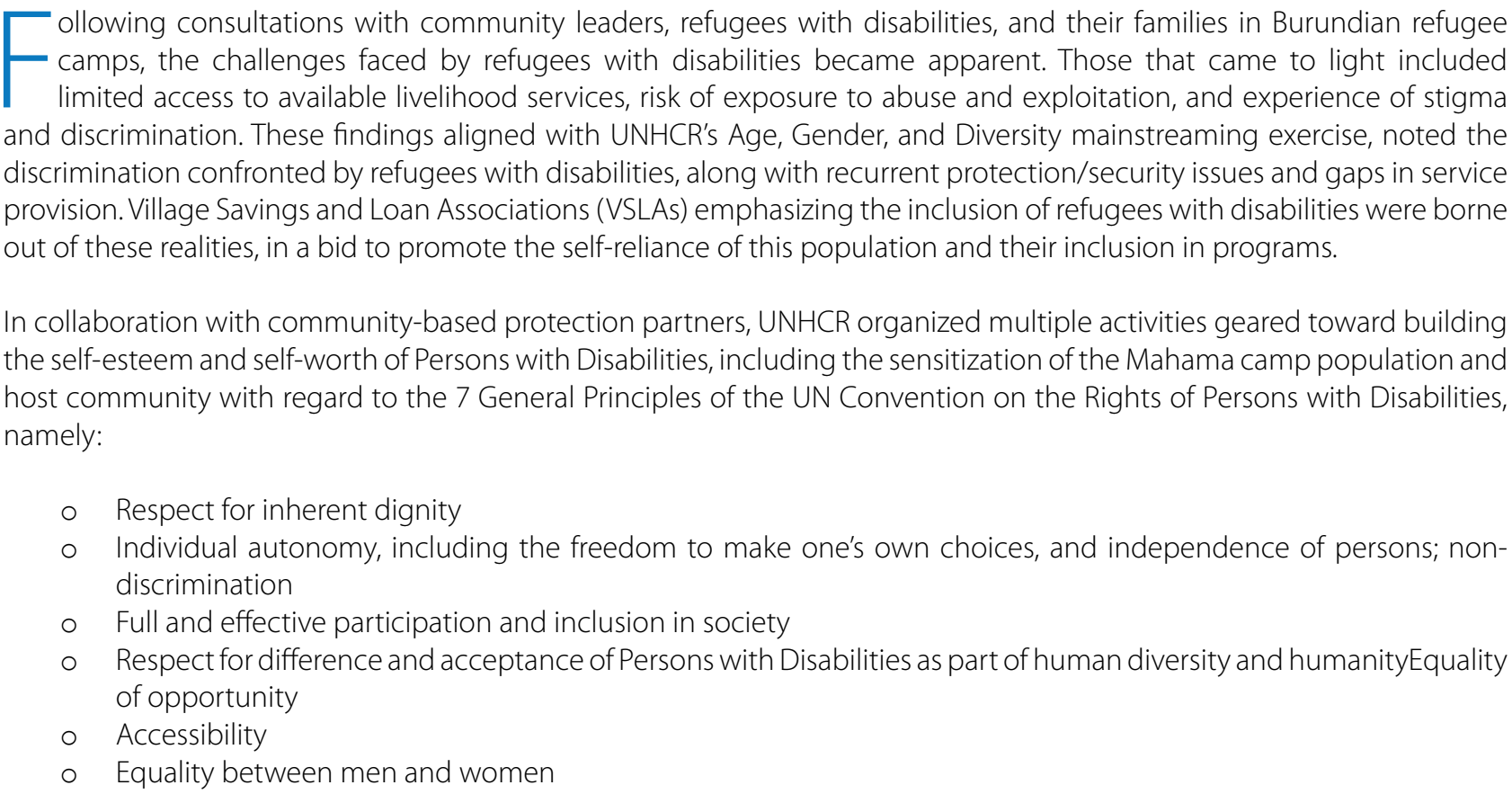

These sensitization sessions enhanced Persons with Disabilities' awareness of their right and freedom to fully participate in the society. Persons with Disabilities therefore formed a disability committee which provided support as participants began developing their own income-generating activities, including Voluntary Saving and Loan Associations (VSLAs), to reduce their aid-dependency and increase their socio-economic participation.

To enable sustainability of the associations created, UNHCR in collaboration with Humanity and Inclusion initiated capacity-building sessions for the associations on the following topics: leadership of the association; participation and the role of each member within the association; means to establish rules and regulations of the associations; the rule of setting goals; monitoring and evaluation within the association; documentation; saving skills; management of savings and loans development; book keeping; and working with financial institutions. The associations were encouraged to open-up accounts in micro-finance institutions in order to enhance their chances of accessing bank loans for durable economic solutions.

UNHCR and implementing partners conduct training sessions and hold regular meetings with the associations, ensuring they are in compliance line with the 'Do No Harm' principle, empowering founding members in a non-discriminatory manner. UNHCR also monitors VSLAs' adherence to agreed-upon regulations, have saving component incorporated into their initiatives, and are proactive about enhancing their self-reliance, self-empowerment and dignity. Regular meetings between implementing partners and beneficiaries, and bookkeeping support, in addition to other strategies, ensure that associations have a monitoring and accountability system in place for transparency.

\section{Results}

- Mahama refugee camp currently has 15 associations mainly formed by Persons with Disabilities, although participation is open to all community members.

- Community members, including leaders, have joined income generating associations initiated by Persons with Disabilities. In Mahama refugee camp, all VSLAs combined make up 361 community members (159 male, 202 female). Of these, 90 are Persons with Disabilities.

- A total of 15 VSLAs have opened bank accounts.

- VSLAs are now sought out by various stakeholders (e.g., the Rwandan government and foreign embassies) to participate in camp competitions, exhibitions, and award ceremonies, and other special occasions. During mass meetings and various international days, time slots are allocated to refugees with disabilities, their families, and partners to share testimonies about the life-changing effects of VSLAs.

- Communities testify that refugees with disabilities also have the capacity and strength to support their families and community at large. 
Partner(s):

Humanity and Inclusion

For further information, contact:

Emilia Lobti: lobti@unhcr.org; rwaki@unhcr.org

\title{
Emerging Practice from Uganda: Enhancing Refugees' Voices through a Refugee Engagement Forum
}

\begin{abstract}
A $s$ the biggest refugee-hosting country in Africa and one of the first to implement the Comprehensive Refugee Response Framework (CRRF), Uganda adopted its CRRF Road Map in January 2018. It set out the vision to strengthen implementation of a comprehensive approach in its response, in order to ease the pressure on host districts and meet the needs of refugees and host communities. Uganda's CRRF Road Map identifies meeting the needs of refugees and host communities as one of the crucial components of the CRRF in the country.
\end{abstract}

The CRRF Steering Group (SG), a high-level decision-making body, meets quarterly to strategically steer CRRF implementation in Uganda. The CRRF SG is a unique platform, bringing together government ministries, departments and agencies, international bilateral and multilateral partners, international and local NGOs, the private sector, refugees, and host communities. Two seats on the CRRF Steering Group are reserved for refugees, who represent 1.44 million refugees in the country.

The Refugee Engagement Forum (REF) was established in October 2018 to ensure the affected communities are now effectively represented at the CRRF SG. Before the REF was established, refugee leadership structures were limited to the settlement level (Refugee Welfare Councils) with no structural engagement at the national level. Due to this limitation, the engagement of the stakeholders with the refugee community at the national level was ad-hoc and not systematic. Currently, the REF is comprised of 37 members from the settlement leadership, reflecting diverse demography, gender and age groups. These include two elected leaders from each settlement, youth representatives, and community leaders in Kampala. The REF members elect two members from the group - one female and one male from different nationalities and different settlements - to represent refugees at the quarterly SG meetings for a one-year duration. The REF meetings are also held quarterly, before each CRRF SG meeting to keep the CRRF SG informed of all decisions that affect refugees. Two elected representatives from the REF have attended all CRRF SG meetings since October 2018. Through the REF, the refugee community is also be kept informed about the discussions and decisions of the CRRF SG.

The REF Taskforce organizes and facilitates the REF meetings. The askforce is composed of the Office of the Prime Minister (OPM), CRRF Secretariat, UNHCR and INGOs (CARE, Oxfam, IRC, and others). OPM's regional Refugee Desks and Settlement Commandants work closely with UNHCR sub- or field offices to support the REF members' work, providing feedback to the community on discussions at REF meetings, and consulting with the community on issues to raise at the REF and CRRF SG.

The Refugee Engagement Forum fosters meaningful participation by the refugee community in the planning, implementation and monitoring of the CRRF in Uganda. The refugee leadership structure is now structurally in place from village - zone - settlement - national (cross-settlement) to share discussions on the ground with the national forum and vice-versa. The REF is not only an important accountability mechanism for all stakeholders in the refugee response; it also contributes to enhancing refugee self-reliance by effectively responding to the needs of refugees and host communities.

Two Refugee Representatives from REF have attended GRF, IGAD, TICAD and other international engagements to share their experience and knowledge and to discuss issues that impact their community.

Partner(s): various

For further information, contact:

Yoko Iwasa: iwasa@unhcr.org

LeslieVelez: velez@unhcr.org

Ndahiriwe Innocent: indahiriwe7@gmail.com

Rick Bartoldus: Rick.Bartoldus@rescue.org

Annet Kyambadde: Annet.Kyambadde@care.org 


\section{Emerging Practice from Uganda: Community-Led Establishment of Refugee Welfare Council Offices, Formation of Persons with Specific Needs Support Groups, and a Skills Development Initiative}

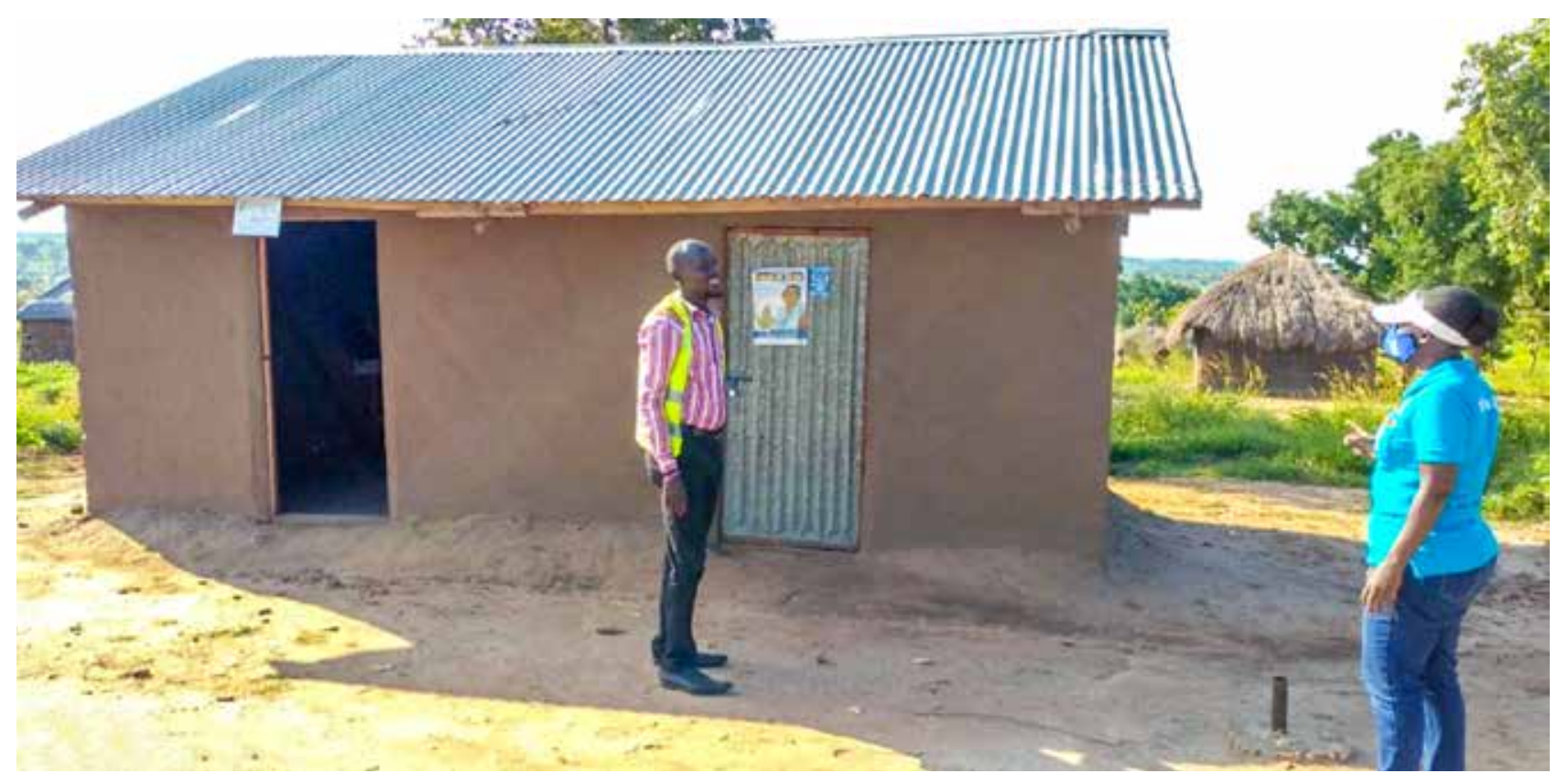

T he construction of semi-permanent Refugee Welfare Council (RWC) spaces in Ofua Zone was a purely communityled initiative spearheaded by the RWC leadership and geared toward enhancing their ability to coordinate more effectively with partners and the entire community. RWC office spaces were viewed as being key to creating an enabling, confidential environment for engagement between community members, partners, and RWCs, while enhancing community participation, maintaining transparency and accountability, and observing existing referral pathways.

RWC office spaces have been established in the 6 Villages of Ofua Zone. These spaces make it easier for refugees and partners to approach RWC leaders for support and involvement in planning, implementation and monitoring of service deliverables. The spaces are open full-time with at least one RWC executive member present to attend to issues, including individual complaints or concerns, feedback-sharing, and the need for meeting space.

In addition, Persons with Specific Needs in Ofua Zone have initiated six functional support groups (one per village), with an additional, seventh group (composed of 19 members and representing the zone) appointed to oversee the six villagebased groups. The Persons with Specific Needs support groups meet regularly to discuss issues that affect them and to develop local solutions to address the issues. Each group consists of a chairperson, vice-chairperson, treasurer, and a secretary who documents group challenges and achievements. The support groups are used as a mechanism for: helping to raise Persons with Specific Needs' awareness of their rights and obligations, providing basic counselling to Persons with Specific Needs requiring it, and engaging Persons with Specific Needs in livelihood opportunities (e.g., poultry farming, kitchen gardening),and in small scale retail business and savings.

Lastly, women in Ofua II village organized themselves into a group of 30 members to train their peers in skills development, including tailoring, beading/knitting/weaving, shoe-making, hairdressing, and soap-making. This women's group offers training to equip non-literate women in the community with skills to enable them to support their livelihood.

\section{Results}

- Persons of Concern have trust in their leadership and continue to present their concerns through the established structures. There is notable participation by the refugee leaders in decision-making to resolve matters that affect them and their community, coupled with greater visibility of leaders in the community through the established spaces. 
- The Persons with Specific Needs support groups have contributed and saved money as part of their Village Savings and Loan Association. They also continue to offer counselling and sensitization services to fellow Persons with Specific Needs on their rights and obligations.

- The women's group in Ofua II has acquired seven tailoring machines and other kits for training other women. Thus far, the group has trained over 16 women in tailoring and 5 women in shoe-making. Ongoing trainings include tailoring (for 10 women) and beading/knitting/weaving (56 women). Group members contribute the equivalent of about Ugx 20,000 for membership, Ugx 500 for registration, and Ugx 1,000 weekly. The group jointly decides how to spend the funds.

Partner(s):

Danish Refugee Council

Office of the Prime Minister

For further information, contact:

Jael Chunge: chunge@unhcr.org

Kalsum ljoru: ijoru@unhcr.org

\section{Case Study from Uganda: Community-Based Peace-Building Initiative}

- ollowing the murder by refugees of a national youth in Rhino Camp refugee settlement (Eden Zone), the relationship - between the refugee and host communities became strained and endangered. It was necessary, therefore, to address and resolve the situation, and to restore the peace that had existed between the two communities prior to the unfortunate incident. The Community-Based Peace-Building Initiative was borne out of these circumstances.

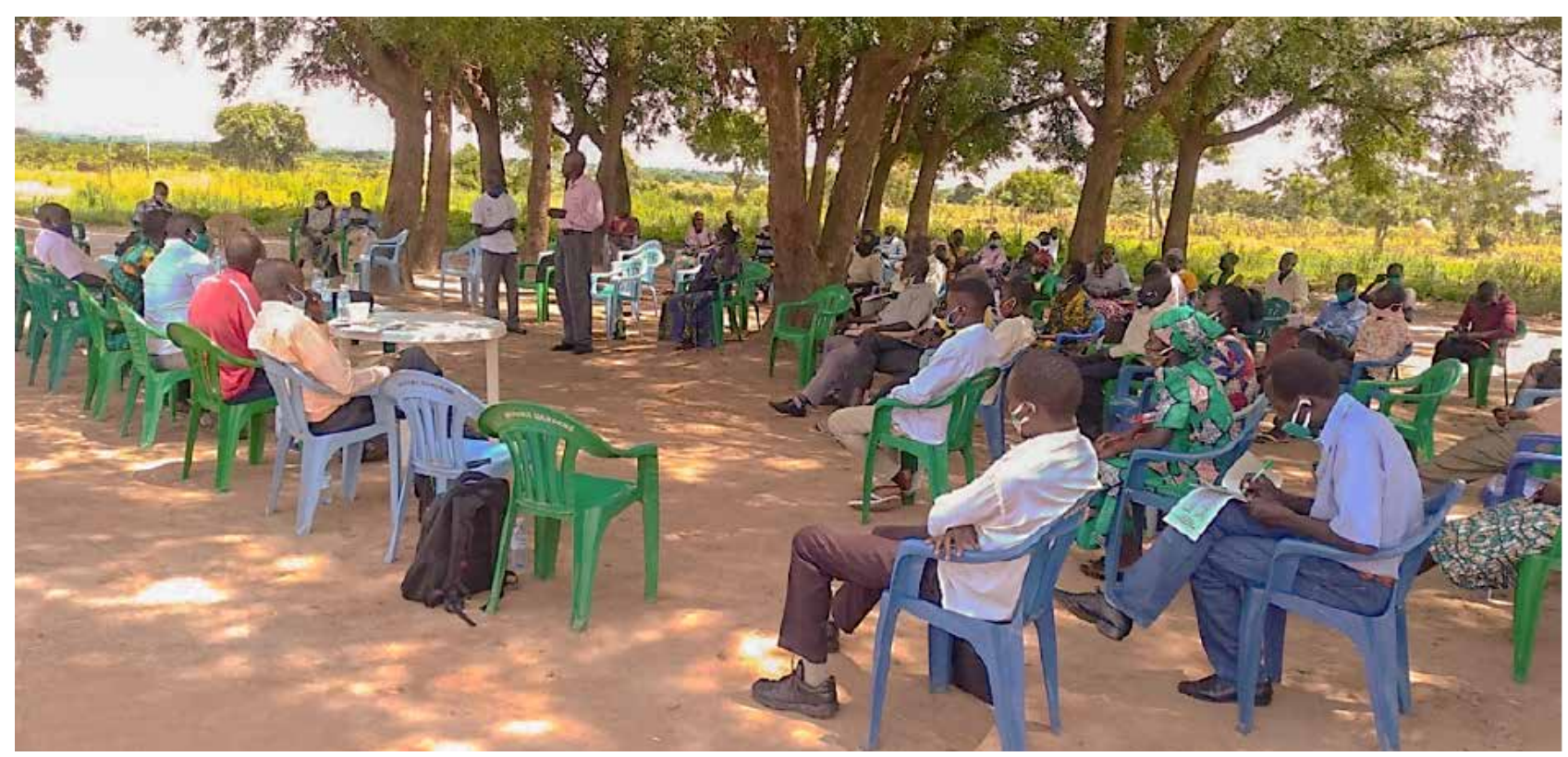

This initiative involved peace-building community dialogues led by Eden Zone refugee leaders and the host community leaders. Four dialogues were conducted - two held with the host community, and two with the refugee community. By the commencement of the dialogues, the suspects had been arrested. During the dialogues, relatives of the deceased decried the crime, while accepting that the condemnation of all refugees would be unfair, and cautioning nationals against retaliation.

The dialogues provided space for refugee community members to express remorse and ask for forgiveness from aggrieved host members. Refugee community members took responsibility for the incident, pointing out to host community members that they had fled their country for their own safety; that murder is unlawful; and that not all refugees were involved in the crime. It was resolved that refugees and nationals should coexist, and continue to share available resources. Refugees who had acquired or hired land were encouraged to resume farming, gathering firewood, collecting building materials, etc. Both communities resolved to put the unfortunate incident behind them and begin a new chapter together. 


\section{Partner(s):}

Community Technology Empowerment Network

Danish Refugee Council

Development Literacy Partner International

Norwegian Refugee Council

Office of the Prime Minister

For further information, contact:

Jael Chunge: chunge@unhcr.org

Ejoyi Geoffrey Bakole: ejoyi@unhcr.org

\section{Emerging Practice from Uganda: Tricycle Initiative}

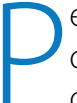
ersons with Specific Needs in Bidibidi Refugee Settlement continue to face challenges in accessing food due to the distant locations of food distribution points (FDP) in the settlement. Some walk up to five kilometers to reach the distribution centers and are forced to sell part of their rations to arrange for collection and delivery. In a bid to lessen Persons with Specific Needs' transport burden, UNHCR introduced a Tricycle Initiative to ensure continued safe and secure access to food assistance for particularly vulnerable individuals among Persons with Specific Needs through an affordable and sustainable transport mechanism.

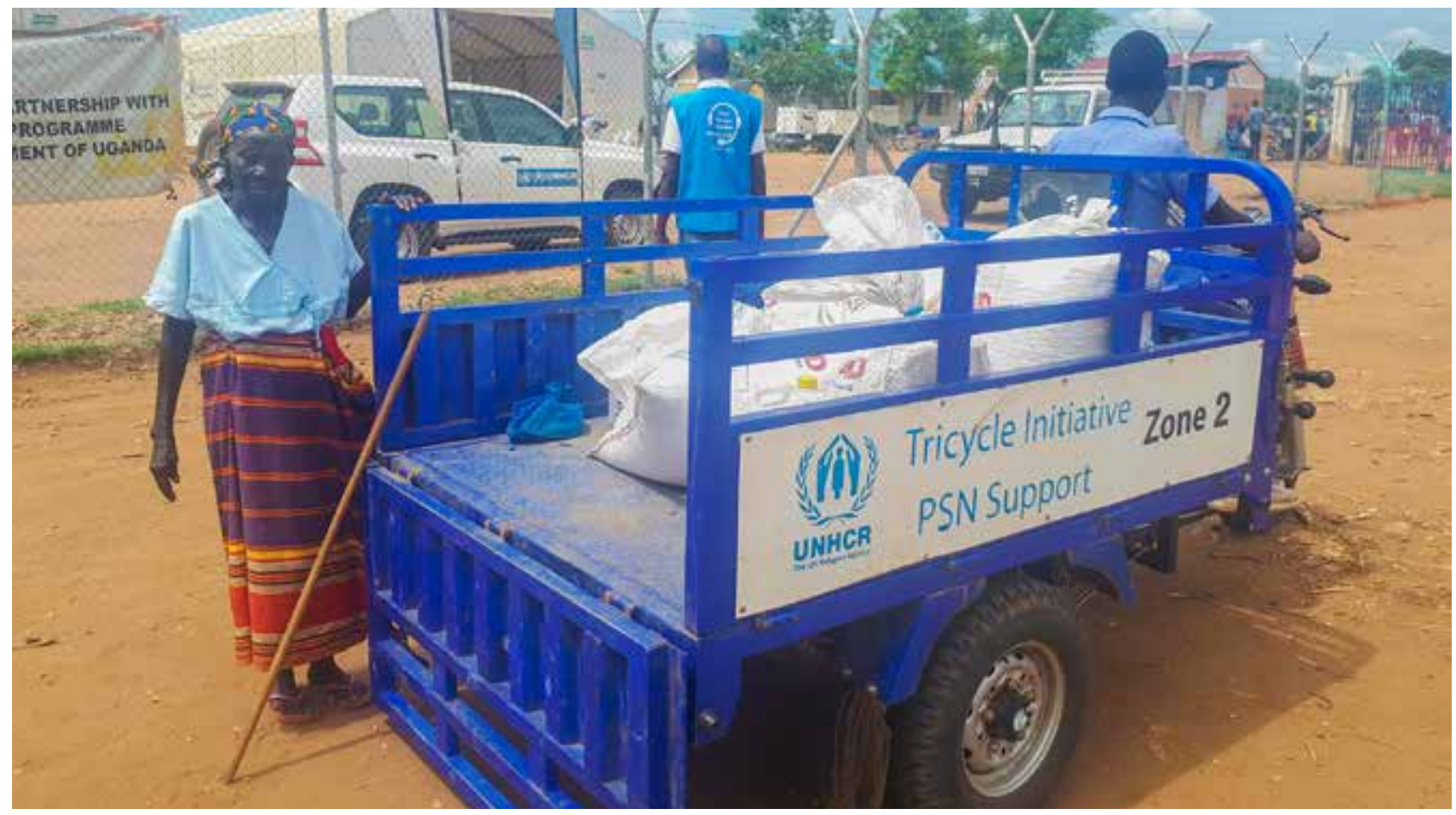

This project has a two-fold objective: 1) to facilitate free of charge transportation services in a safe manner for particularly vulnerable families from FDPs to their homesteads within the settlement, and 2) to support income-generating opportunities to selected Village Savings and Loan Association (VSLAs) on non-food distribution days.

To accomplish these objectives, UNHCR allocated 20 motor-tricycles to Bidibidi Refugee Settlement. The tricycles were distributed per zone to selected VSLAs, depending on zones' needs. The allocation was done taking the size and typology of the zone into consideration, along with the distance from FDPs, and the number of particularly vulnerable individuals in need of transport in each zone. Protection partners helped identify such individuals through a community-based approach, engaging the community structure (RWC Persons with Specific Needs secretary). The VSLAs were also selected from among more than 1,000 active groups through an inter-agency approach involving all protection and livelihood partners as well as the Office of the Prime Minister (OPM).

The groups were selected by assessing their experience, the level and evidence of their savings and loans transactions, as well as the group's motivation to serve Persons with Specific Needs, which was determined by interviewing all the 


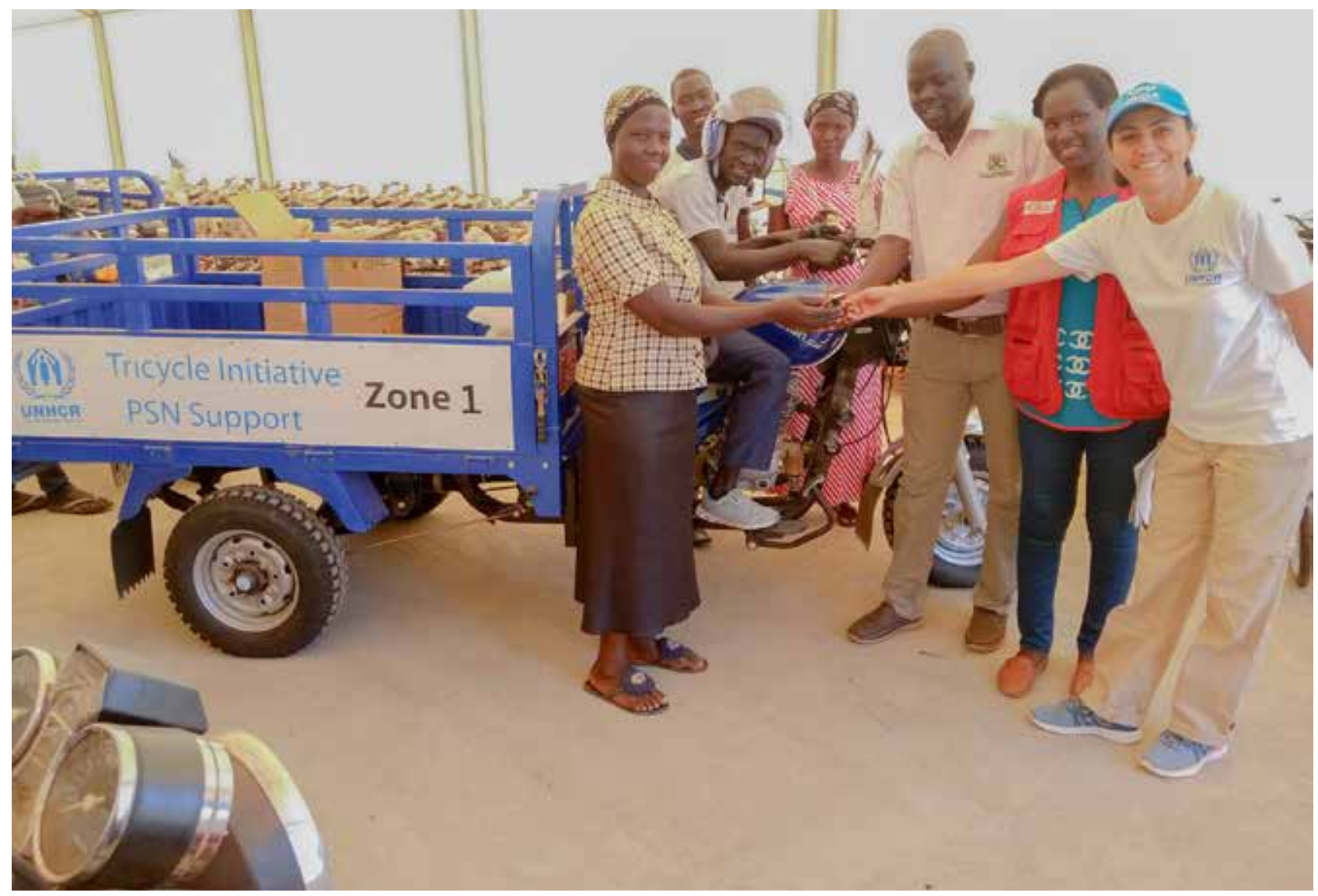

members of the group.Some groups are gender-specific, while others are mixed-gender, depending on the business model of the VSLA, but a mix of both males and females were among those selected for the initiative.

The selected VSLAs were trained to operate the tricycles and two members per group were provided with riding permits. The training and riding permits were facilitated by Dan Church Aid. The VSLAs were supported with fuel and minor repairs and service for an initial period of three months, giving them time to become self-reliant. An MOU was signed between each VSLA, DCA, UNHCR, OPM and IRC, stipulating the objective of this initiative and the roles and responsibilities of all parties.

The 20 tricycles were handed over to the groups in different zones of Bidibidi settlement. They are working well, supporting particularly vulnerable individuals during food distribution in their respective zones to transport their foods free of charge to their homesteads, for an average of five working days per distribution. During the rest of the month, they engage in activities for income generation within the terms and conditions of the MOU that they signed. The initiative has had a positive effect on income generation of the VSLAs.

\section{Results}

The following quote from a VSLA member encapsulates the results garnered by the initiative so far: 'When we started with the tricycles, in our weekly sittings. the members were saving UGX 3,000. We are planning to increase the saving to UGX 4,000 for our next annual cycle which starts October 2020, nine months after start of the Tricycle Initiative. Despite the situation of coronavirus and restriction of movements, we still manage to get jobs around the settlement and the project increased the VSLA income generation significantly. Before, the highest amount of loan was UGX 500,000. However, we can now give out loans up to UGX 1,000,000. This will help the members to think of bigger projects and exponentially increase their possibilities.'

Partner(s):

Dan Church Aid

International Rescue Committee

Office of the Prime Minister

For further information, contact:

Elham Baghdadi: baghdad@unhcr.org

Vincent Bukenya: bukenyav@unhcr.org 


\section{Case Study from South Sudan: Community-Based Protection Network for IDPs}

\footnotetext{
7 he outbreak of conflict in South Sudan led to the displacement of many people into urban centers. Despite a signed peace agreement, there is still fear among displaced populations. Many internally displaced people (IDPs) fear for their lives and are reluctant to return to their homes. The majority of the IDPs were left vulnerable after their homes were vandalized and their livelihoods were disrupted as a result of armed conflict.
}

To support IDPs, a Community-Based Protection Network was established as a key protection structure which would promote community engagement on humanitarian assessment or distribution, identification of vulnerable households in the IDP communities, and information-sharing in 9 IDP sites in Yei Town. The new community-based structure was established, while strengthening existing local structures as a Protection initiative. This approach was geared toward ensuring community ownership and sustainability of the initiative. The Community-Based Protection Network provides a forum for community members to help each other with everyday work needs, and to develop and disseminate best practices, guidelines, and strategies for their members' use.

Under this Network, continuous on-the-job training for community structures was conducted to enhance skills and improve the quality of support provided to the Vulnerable IDPs and host communities. To maximize the use of available resources, and avoid double-targeting and the duplication of effort, the United Methodist Committee on Relief has collaborated with other humanitarian actors throughout this initiative. Proposed project activities were conducted within 9 established IDP sites with the help of the State government. The established structures played a major role in conducting community sensitization and awareness, identification of beneficiaries, reporting of protection violations, and in responding to affected victims who sought support and justice for atrocities committed.

The Community-Based Network has led to the following:

- Functional community-based protection monitoring system, resulting in increased awareness of SGBV risks, clear reporting channels and timely and dignified case management procedures for SGBV survivors

- Functional and efficient data collection mechanism with validation and respect for confidentiality.

- Functional established support groups for Persons with Specific Needs to ensure community self-protection and self-management

Partner(s):

United Methodist Committee on Relief

Mission to Alleviate Suffering in South Sudan

For further information, contact:

Wamutshilani Nkala: nkalaw@unhcr.org 


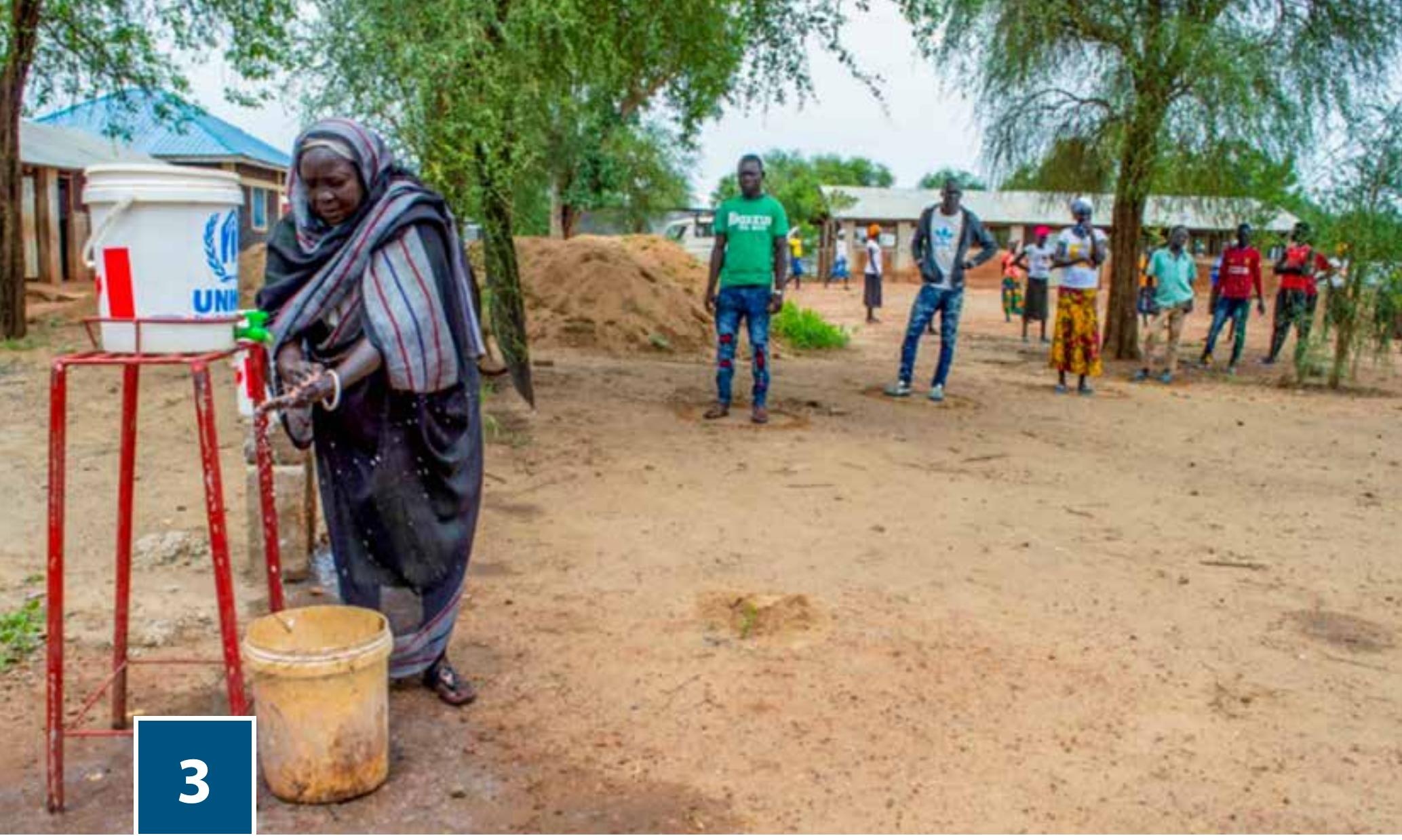

\section{GENDER EQUALITY}

\section{Promising Practice from Ethiopia: Refugee Outreach Volunteers in Addis Ababa}

1 hile the majority of refugees in Ethiopia reside in refugee camps, approximately 30,000 refugees of different $\sqrt{ }$ nationalities reside in the capital city of Addis Ababa. Some refugees come to Addis Ababa through referrals from the refugee camps for health, security, SGBV and other humanitarian reasons that cannot be addressed in the refugee camps. The language barrier in urban centers often poses barriers for refugees in accessing various national services, including legal, health, education, and other services provided by the government.

Refugee Outreach Volunteers (ROVs) are volunteers who reach out to fellow refugees with specific needs (such as older persons, person with disabilities, SGBV survivors and LGBTI) and provide them with accurate information, conduct awareness-raising on important issues, and assist in identifying and referring refugees to appropriate service providers for any protection, health, registration and other needs. Given their familiarity with their respective cultural, social-economic and religious contexts, and strong understanding of refugees' protection needs and gaps, ROVs also play a key role in preventing and addressing misinformation and misunderstanding by the refugee population about UNHCR and partner services.

The intervention approach entails selecting ROVs from the community in a structured, inclusive, and transparent manner, taking AGD dimensions and the ROVs' standing and networks in the community into consideration, with the technical support of UNHCR and the government counterpart, Agency for Refugee and Returnee Affairs. A total of 49 ROVs (24 males and 25 females) were selected from Great Lakes, Sudan, Yemen, South Sudan, Eritrea, Somalia, and other nationalities living in Addis Ababa. With technical guidance from UNHCR, ROVs are divided into three thematic areas of child protection, general protection, and health, and carry out a range of tasks to identify refugees with specific needs. These tasks range from locating foster families on behalf of separated and unaccompanied children, and supporting patients with translation at health facilities, to accompanying such patients to service providers in other sectors, including the police and legal sectors, as needed.

ROVs receive intermittent with training and refreshers on'Do no harm' principles, confidentiality, inclusivity, and impartiality. They are evaluated on these issues periodically during monthly supervision visits, and their commitment is reviewed during this time, as well as during monthly case management meetings and quarterly general ROV meetings that provide 
a platform for all ROVs to share their challenges and success, and build their team spirit. The training of ROVs covers basic Protection from Sexual Exploitation and Abuse concepts and anti-fraud, and each ROV signs a Code of Conduct. ROV s are assigned to identify four cases per month consisting of Persons with Specific Needs who require UNHCR or an implementing partner's attention. Identified cases are followed-up on, with feedback on actions taken provided to ROVs.

\title{
Results
}

Results are measured based on monthly reports of Persons of Concern reached, referred to service providers, number of follow-ups made, and needs met. Feedback from Persons of Concern and immediate supervisors is recorded and filed in each ROV physical file as well in the UNHCR ProGres database.

\section{Partner(s):}

Agency for Refugee and Returnee Affairs

Development and Inter-Church Aid Commission

Jesuit Refugee Service

For further information, contact:

Nunu Aliyi: aliyi@unhcr.org

\section{Case Study from Kenya: Gender Equality in WASH Provision}

W

ASH services in Kakuma refugee camp and Kalobeyei settlement are specifically organized to be provided to all Persons of Concern - including women, men, girls, and boys - in equal and equitable measure. Special emphasis is placed on women and girls as they are more likely to be left behind and underrepresented in conventional community participation approaches.

At the camp level, WASH support beneficiaries are identified through multifunctional teams composed of UNHCR, the Refugee Affairs Secretariat, Health implementing partners, and a range of other partners. Women-headed households are given priority. At the block level, WASH Committees (community WASH representative structures) are trained to identify and coordinate WASH issues within their respective blocks. The Norwegian Refugee Council has ensured diverse leadership representation within WASH Committees, including a 50:50 ratio between male and female representatives. Female candidates are prioritized in the recruitment of WASH community outreach staff as well.

Partner(s):

Norwegian Refugee Council

For further information, contact:

Moe Miyahara: miyahara@unhcr.org

Oscar Nabiswa: nabiswa@unhcr.org

JohnWagacha Burton: burtonj@unhcr.org

\section{Case Study from Uganda: Age, Gender, and Diversity Workplan/Indicator Table}

\begin{abstract}
A pplying an Age, Gender, and Diversity (AGD) approach in our work is essential to ensure that our interventions are safe and accessible for everyone and are provided without discrimination. The approach involves considering the distinct needs and views of women, men, girls and boys of diverse backgrounds, including Persons with Disabilities, persons with diverse sexual orientations and gender identities and ethnic, religious and linguistic minorities or indigenous groups, in assessment, planning, implementation and monitoring processes.

In order to ensure that all UNHCR colleagues and sectors apply an AGD approach in their work, the Community-Based Protection (CBP) and Program teams developed national AGD Action and Workplans for the operation. The current AGD Workplan is based on the national AGD Action Plan that was developed in 2019. The national AGD Action Plan consolidated the field action plans which were developed after a deep dive workshop in 2018, and contains a list of challenges, field activities and national level activities to effectively implement the UNHCR AGD policy. This action plan was further developed through consultations with sector leads in Kampala, field-based protection staff and partners.
\end{abstract}


The AGD Workplan was based on the AGD Action Plan, but resembles an indicator table, introducing outcome and output level indicators, baselines and targets. This will help us to more effectively monitor the implementation of the activities in the work plan. The work plan/table will be finalized through further consultations with sector leads (in and outside of UNHCR) and field colleagues. This has been a joint effort between CBP and Programme colleagues.

The impact of the AGD Workplan for 2020-2021 is yet to be assessed. However, it is expected to have an impact on the way services are delivered in Uganda, and on how UNHCR's AGD policy is implemented. The effective application of an AGD approach in partner's work would increase safety and meaningful access to services for all and empower marginalized and disenfranchised groups.

The process of working with partners and UNHCR staff has already greatly increased awareness of the policy. The partnership between Protection and Program has been very positive. The involvement of a Senior Program Officer has made it a UNHCR initiative, rather than a Protection initiative.

Partner(s): various

For further information, contact:

Sam Mosallai: mosallai@unhcr.org

Yoko Iwasa: iwasa@unhcr.org

Clare Askew: askew@unhcr.org

\section{Case Study from Uganda: Age, Gender, and Diversity Workplans for Implementing Partners}

\footnotetext{
$\Lambda$ n Age, Gender, and Diversity (AGD) approach promotes a nuanced consideration of the realities, views, and needs of women, men, girls, and boys of diverse backgrounds in assessment, planning, implementation, and monitoring processes. Populations of interest also include Persons with Disabilities, persons with diverse sexual orientations and gender identities and ethnic, religious, and linguistic minorities or indigenous groups.
}

To foster the application of an AGD approach in the work of its implementing partners, UNHCR made compliance with its AGD Policy a mandatory requirement for all implementing partners. The Community-Based Protection (CBP) team, with the support of Program colleagues, has since been working with new implementing partners to build their capacity on the AGD approach. Prior to this capacity-building exercise, UNHCR's existing Project Partnership Agreement (PPA) templates contained sparse reference to $A G D$, with no mention of the expectation that implementing partners should comply with UNHCR's AGD Policy. Consequently, partners were not aware of the policy.

To rectify this situation, the CBP team worked closely with Program colleagues throughout the 2020 partner selection process. CBP colleagues attended all introductory meetings with new partners, gave presentations on the AGD policy, informed partners of their obligation to comply with the policy, and provided them with AGD support packages. Furthermore, detailed reference to the AGD approach was included in all new PPAs and cover letters, and it was agreed that all partners would develop individual, sector-specific AGD workplans. Additionally, a half-day training session was conducted, during which partners were further sensitized on the AGD policy, as well as the Inter-Agency Standing Committee Gender with Age Marker. Partners also received guidance on the development of sector-specific partner AGD workplans for the following year, to ensure the effective mainstreaming of Gender Equality (GE) and Accountability to Affected People (AAP) in all partner activities. These workplans contain activities, indicators, focal persons, and deadlines. Progress made towards the implementation of the workplans will be monitored during mid-year and end of year performance reviews.

The impact of AGD inclusion in the PPAs for 2020, the introduction of mandatory reporting requirements, and the development of partner sector-specific AGD workplans are expected to have an impact on service delivery in refugee settings in Uganda. The effective application of an AGD approach in partners' work will likely increase safety and meaningful access to services for all, and empower marginalized and disenfranchised groups. This collaborative process between UNHCR staff and partners has already greatly increased awareness of the AGD policy.

Partner(s): various

For further information, contact:

Sam Mosallai: mosallai@unhcr.org

Yoko Iwasa: iwasa@unhcr.org 


\section{Results}

The refugee and host communities alike now have access to a low-cost, conveniently located grinding mill. The location of the mill within the refugee community reduces protection and security risks for women, girls, and youth. The fully selfreliant Women Association has generated profits, which has led to a decision by the Association to include more women from the refugee community, and to expand the project by venturing into beauty parlor and catering business initiatives in the camp. The business expansion program is currently under study by the Women Association. The grinding mill has also created salaried job opportunities for more than 4 Persons of Concern within the camp. Women involved in the project have raised their livelihoods and are contributing financially to their households and families. The project has the gained attention of more women who are seeking participation in such livelihood projects.

Partner(s):

Agency for Refugee and Returnee Affairs

For further information, contact:

Alisona Rajbanshi: rajbansh@unhcr.org

\section{Case Study from Kenya: Gender-Sensitive WASH Strategies as an SGBV Prevention Measure}

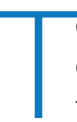
o deter SGBV perpetrators in refugee camps, water points are deliberately constructed in open spaces and within community reach. Furthermore, water supply is delivered from 7 am to 5 pm, rather than later in the evening or night, to ensure the overall safety of the community. To mitigate conflict at the water points (tap stands), WASH committees and refugee leaders have worked with their communities to develop a community water-fetching schedule organized around morning, noon, and evening periods.

Additionally, communal latrines for new refugee arrivals are designed with internal shutters to protect privacy and dignity. Community members have also been sensitized to accompany minors and women to the latrines at night.

Partner(s):

Norwegian Refugee Council

For further information, contact:

Moe Miyahara: miyahara@unhcr.org

Oscar Nabiswa: nabiswa@unhcr.org

John Wagacha Burton: burtonj@unhcr.org

\section{Emerging Practice from Kenya: Community-Run Safe Homes}

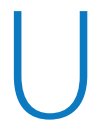
NHCR and partners have a pool of trained volunteers from the refugee community who have opened up their homes to provide safe spaces for survivors of SGBV in need of temporary shelter and support pending the assessment of their needs and solutions. Trained volunteers provide shelter within their household space and assist with the referral of survivors to key service points, such as health facilities. This intervention ensures that survivors who do not require long-term institutionalization still have access to community-based safe spaces, and that the community's capacity is strengthened to independently identify solutions and support.

SGBV survivors receive material support, psychosocial first aid, referrals to services on an emergency and voluntary basis. Only low- and medium-risk cases are admitted into community safe homes after a safety assessment and safety planning exercise are conducted with consent of the survivor. A survivor-centered approach is emphasized while considering admitting survivors into community safe homes. The views of the safe home volunteer in relation to the peculiar profile details and requirements of the case are also taken into consideration to ensure harm is avoided for both the survivor and community volunteer. Regular interagency coordination meetings and ad hoc case conferences are held to determine case plans and track progress throughout the admission process. 


\section{Results}

Community safe homes have been embraced as a supplement and substitute for institutional safe spaces, as such services are often limited or lacking. The program has also been used to provide safe shelter for men and boys, and persons of diverse Sexual Orientation, Gender Identity and Expression - populations for whom no safe spaces are dedicated to their protection, and for whom access to emergency shelters poses a challenge.

The community itself has been able to respond to cases that require urgent safety and safe shelter pending discussions with SGBV case workers. Community identification and referral of SGBV incidents has also been strengthened, with the safe home volunteers acting as focal point persons that the community can engage.

\section{Partner(s): $\mathrm{n} / \mathrm{a}$}

For further information, contact:

Aisha Ngonze: ngonzea@unhcr.org

\section{Emerging Practice from Kenya: Community Caregivers - Temporary Urban Safe Shelters for SGBV Survivors}

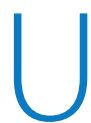
NHCR and the Hebrew Immigrant Aid Society (HIAS) have worked with community structures in urban refugee settings to cultivate a pool of refugee volunteers who offer their own homes as safe, temporary accommodation for SGBV survivors. In the Nairobi neighborhood of Kayole, these community volunteers/caregivers receive at least two trainings annually to boost their capacity to support SGBV survivors. They also receive a stipend to support their hosting costs. Partner agency social workers monitor the household shelters and provide further support to both the survivor and caregiver.

Community committees are sensitized on the community caregiver initiative, and serve as a platform for identifying and vetting community caregivers prior to their enrolment in the program. Where no community caregiver is available take in a survivor, other community structures (e.g., churches) are used as safe havens for survivors.

\section{Results}

The practice is a community-based solution which underscores the fact that communities can support SGBV survivors outside of the conventional partner referral pathway, which may not be functional at critical times (e.g., at night). The intervention has resulted in timely response to survivors, including ensuring their access to safe shelter, health facilities and police stations, as well as immediate counselling.

Community caregivers have developed strong working relationships with the authorities, particularly, the police and sub-county government officials, resulting from their regular interactions with these stakeholders as they work to support survivors in their households. Other community members who are not within the pool of community care givers also occasionally volunteer to host SGBV survivors.

Partner(s):

Hebrew Immigrant Aid Society

Refugee Affairs Secretariat

GBV Working Group

Other SGBV partners

For further information, contact:

Jackson Karugu: karugu@unhcr.org

Daniel Mureithi: mureithi@unhcr.org 


\section{Emerging Practice from Rwanda: Teenage Pregnancy and School Dropout Initiative}

\footnotetext{
- eenage pregnancy is a longstanding problem in Kiziba camp, increasing the risk of school dropout among teenage girls. From March to August 2020, 22 female students were identified as being pregnant and were at risk of dropping out of school.
}

A number of factors drive school dropout for pregnant students in the camp, including a lack of family/social support for reintegrating affected girls into the education system; fear of discrimination from teachers and peers in school; and the lack of a timely risk mitigation structure for school dropout in the event of teenage pregnancy. The current initiative therefore aimed to: assess the challenge of school dropout through a mapping exercise; understand the risks of school dropout for pregnant teenage girls via focus group discussions; strengthen family and social support for pregnant teenage girls to promote their reintegration into the education system, as an SGBV intervention strategy; and, ultimately, ensure a smooth transition back into the education system.

The practice comprised the following components:

- A comprehensive out-of-school children mapping exercise, facilitated by refugee teachers and the UNHCR education partner, through which 185 children ages 3-19 were identified as having dropped out of school for multiple reasons.

- Focus group discussions with girls, boys, parents, and teachers to better understand the risks for school dropout by pregnant girls. Girls explained that they felt their lives were over once they found out they were pregnant. Their parents felt too ashamed to let them return to school, and the girls worried that they might face discrimination at school.

- The establishment of a community structure consisting of refugee teachers and CPCMs to organize home visits and provide counseling to the families of pregnant girls identified by school authorities. This community structure was established in collaboration with Education and Child Protection units, as well as with the Karongi CBP team.

- Impending training sessions for teachers and CPCMs (led by the SGBV partner and health partner) to strengthen knowledge and communication capacity in risk mitigation.

\section{Results}

With continuous effort, in the year of the intervention commencement, 15 teenage girls who had dropped out of school due to pregnancy returned to school, and some of them performed excellently in national examinations.

\section{Partner(s):}

World Vision International

Refugee teachers

\section{For further information, contact:}

Ruizhi Zhu: zhur@unhcr.org; rwaki@unhcr.org

Speciose Buwamaria: buwamari@unhcr.org

\section{Promising Practice from Rwanda: Survivor Identification and Response through Screening}

\footnotetext{
A Ithough SGBV is known to be a common problem in refugee settings, under-reporting is a challenge which prevents SGBV survivors from receiving the multi-sectoral response that they need. To foster the proactive identification of SGBV survivors and to connect them to comprehensive care in a convenient manner, UNHCR Rwanda adopted an evidence-based SGBV response model developed by the Population Council and Kenyatta National Hospital, Kenya, and evaluated in refugee settings in Uganda in collaboration with UNHCR.
}

The intervention involves the following components:

- Provider Training and Planning Session: UNHCR and the Population Council trained healthcare providers on SGBV screening techniques, and collaboratively adapted a tested screening tool used to detect current and past experiences of SGBV, including (physical, psychological, and sexual) intimate partner violence and lifetime, nonpartner rape. The training also centered on core SGBV concepts, values clarification, screening ethics, survivorcentered approaches, supportive referrals, and documentation. Training participants included providers from 5 


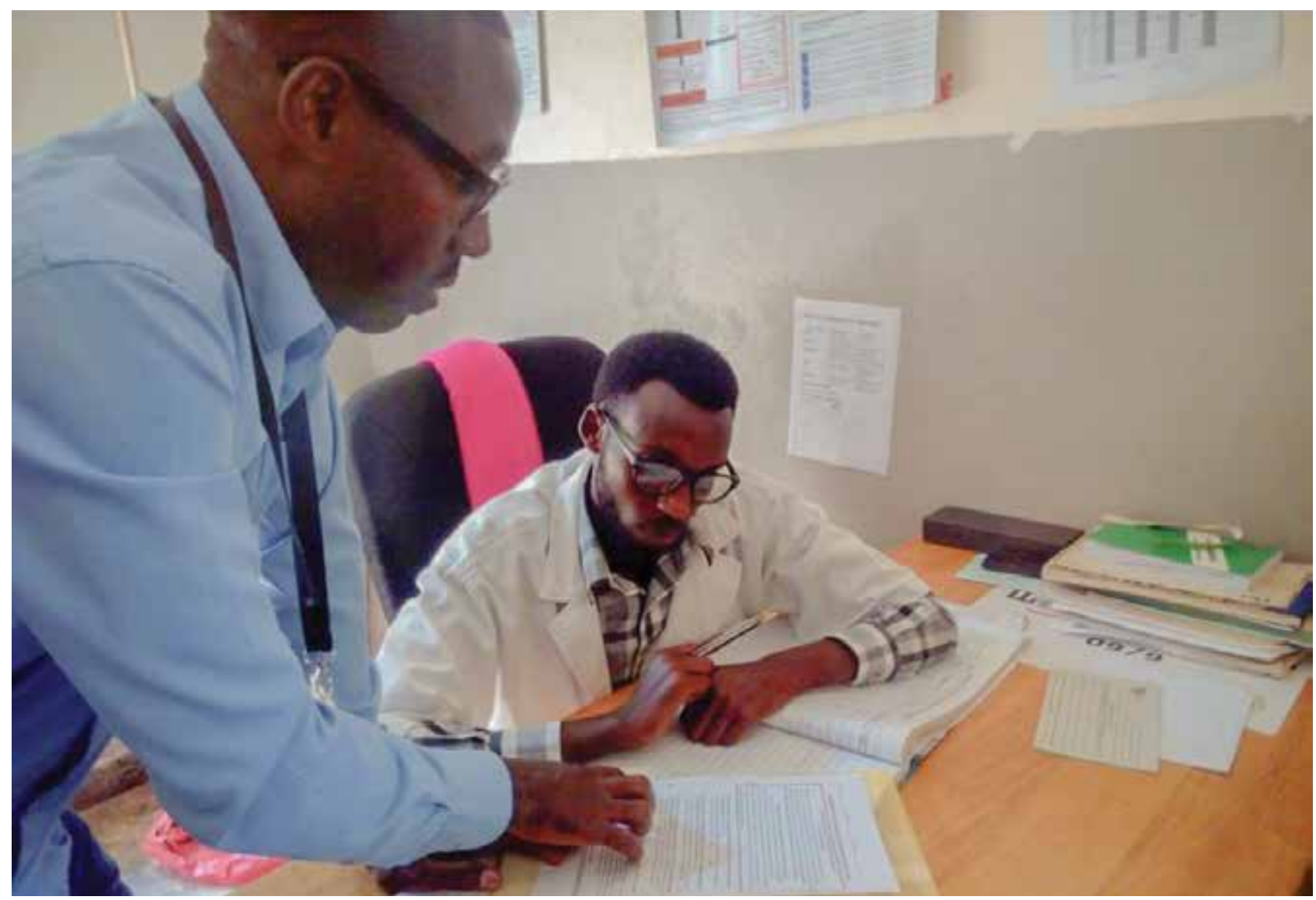

camps in Rwanda, representing departments/clinics where audiovisual privacy could be assured (specially, the Outpatient, Non-Communicable Diseases, Inpatient, Antenatal Care, Maternity, HIV, and Nutrition Departments). The session was also used as a forum for working collaboratively with provider participants to adapt the entire screening process to the realities of their health facility contexts.

- Screening and Referral: Trained healthcare providers (nurses, nutritionists, social counselors, midwives) administered the brief screening tool in the context of private, one-on-one consultations with all female clients aged 14 and above presenting for health services in the respective clinics/departments. Clients found to be 'SGBV-positive' via the screening tool were referred (with their consent) to SGBV comprehensive care centers available on-site in all participating health facilities. To strengthen referrals, phone calls between providers were used where accompanying survivors to the SGBV care centers was not possible.

- Data Collection: Participating clinics use 2 monthly reporting templates to capture the number of: clients screened, survivors identified, survivors referred for comprehensive care, and survivors receiving care at SGBV comprehensive care centers. Incidents by type of SGBV, observations, challenges, and needs are also captured, and data is disaggregated for women and girls to help enhance programming.

SGBV screening in refugee camps in Rwanda started in August 2017 in Gihembe and Nyabiheke camps, and in July 2019 in Mahama, Kigeme and Mugombwa Camps, and has been ongoing ever since. Health care providers working in these camps are trained to screen female clients routinely to detect as many survivors as possible as early as possible.

\section{Results}

- In 2017, participating health facilities in 2 Rwanda camps experienced a 300\% increase in the proportion of survivors identified and connected to comprehensive SGBV care within 2 months after the commencement of the screening intervention.

- In 2019, in the 5 participating refugee camps in Rwanda, 326 persons were identified as SGBV-positive via screening, and 276 were referred to SGBV response services.

- In 2019, participating health facilities in refugee camps in 4 countries combined (including Rwanda) experienced a 129\% increase in the proportion of survivors identified and connected to comprehensive SGBV care within 6 months of the commencement of the screening intervention.

- In the 5 Rwanda camps where routine SGBV screening is being conducted, SGBV response partners report a sudden increase in cases received, which is thought to relate to the significant increase in SGBV survivor detection and access to care. Qualitative data collected from both clients and providers at the health center indicate that women and girls are now aware that, in addition to the SGBV response partner/agency, they can also report SGBV 
incidents at the health center. This has contributed to the number of detected cases of SGBV.

- In addition to Rwanda, the intervention has been successfully replicated by UNHCR and its implementing partners in South Sudan, Uganda, and Zambia.

- In collaboration with the Population Council, UNHCR has conducted an initial set of meetings with education partners, district, teachers and students, to prepare the ground for introducing a child sexual abuse screening intervention in schools in Mahama, Mugombwa and Kigeme camps. This initiative, also initially developed and evaluated in Kenya by the Population Council and Kenyatta National Hospital, is pending due to COVID-19.

\section{Partner(s):}

Africa Humanitarian Action

Alight

Plan International

Population Council

Save the Children International

\section{For further information, contact:}

Zahra Migrani: mirghani@unhcr.org

\section{Case Study from Kenya: \\ Chain Management of Forensic Evidence}

A s part of a multi-sectoral response to SGBV, access to justice/legal services is available to refugee survivors according to their needs and choices. However, prosecutions are often hampered by a lack of forensic evidence for rape and physical assault cases. Consequently, some cases end up being dismissed from courts of law. Police departments in Kibondo and Kasulu Districts have Forensic Units and are keen to provide support to refugee camps. Gaps exists within camp contexts, however, including the lack of sexual assault/post-rape care (PRC) kits across all camp health facility locations, the need for PRC form standardization, inadequate documentation of police medical examination report forms ('PF3'), and poor collection of forensic evidence specimens.

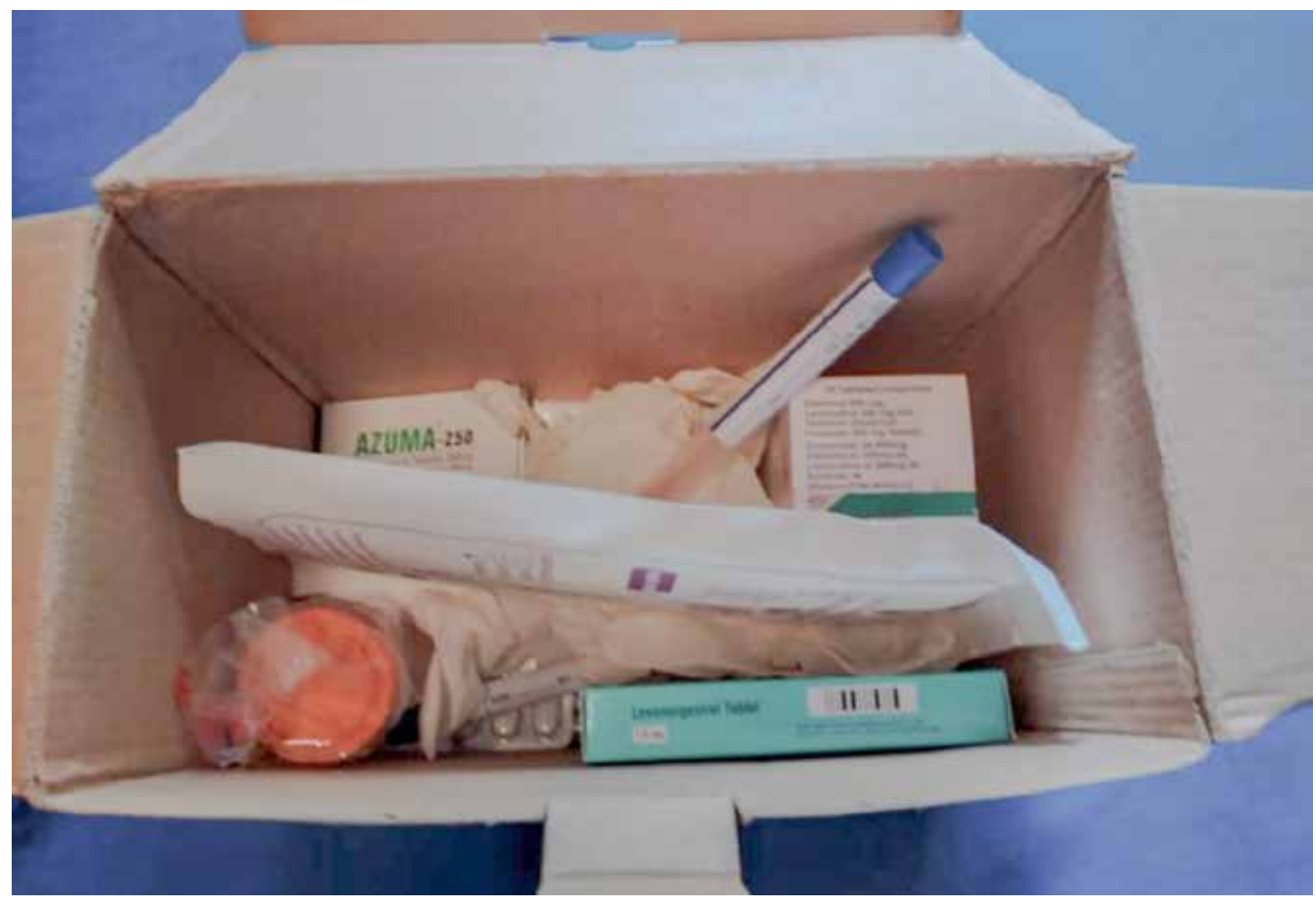




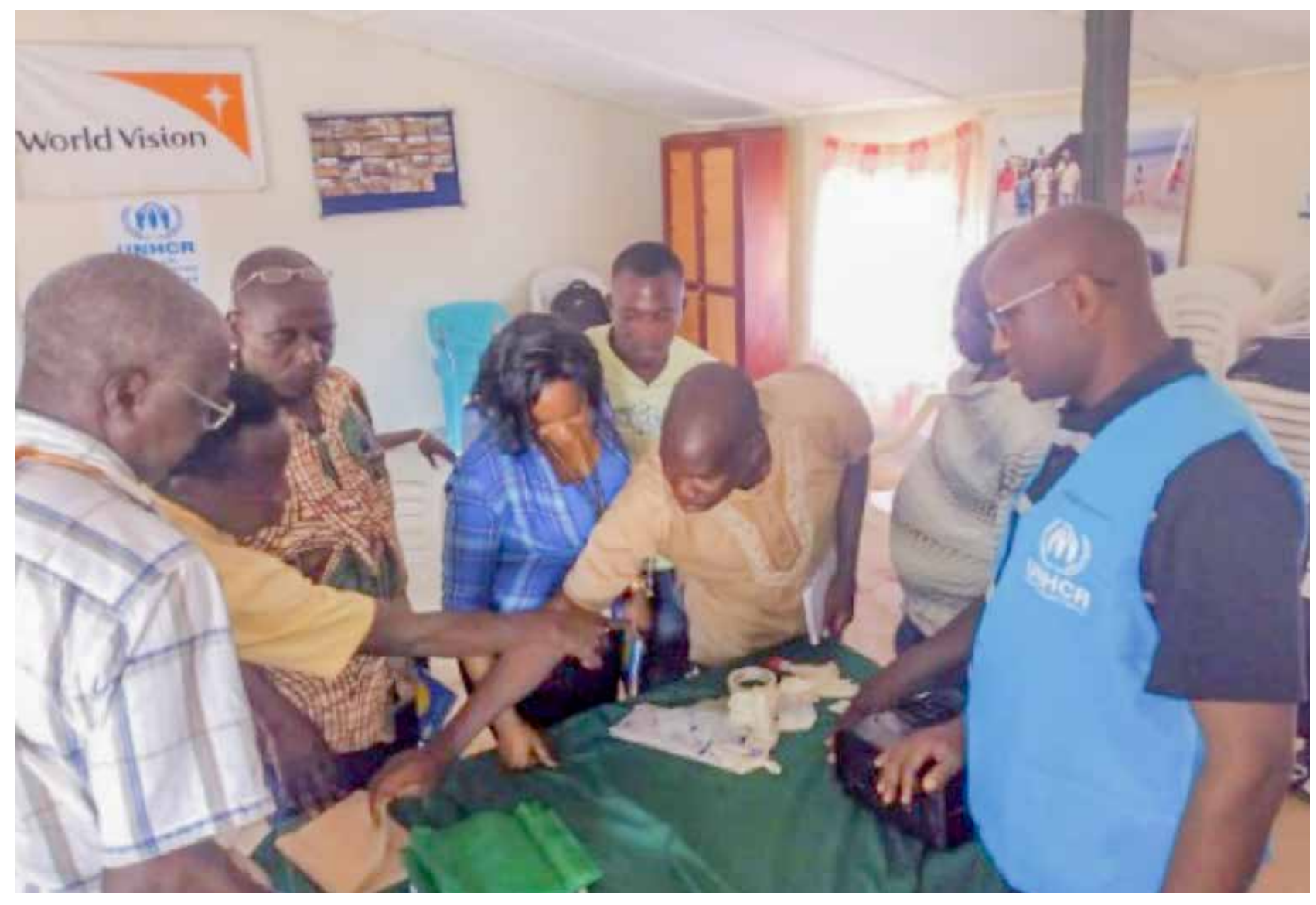

In police stations, there is a lack of forensic police investigators in all camp locations, and a lack of funds for processing forensic evidence from the scene to the government chemist. In general, coordination among police, protection and health actors is weak, coupled with a lack of equipment for collecting and analyzing forensic specimens at SGBV Support Centers.

In response to these realities, the current intervention aims to strengthen the chain management of forensic evidence collection by building the capacity of post-rape care health and police providers to collect, document, and use forensic evidence. The intervention was originally developed by LVCT Health, Kenya, and entails two main components: the assembling of a PRC kit, using material already available in health facilities (albeit in various locations within these contexts); and multi-sectoral training for PRC health, police, and legal/justice providers on national PRC documentation forms that are meant to be filled out collaboratively by health facilities and police stations when responding to rape cases. This documentation needs to be filled out accurately to facilitate the prosecution of rape cases in court. As the documentation forms (post-rape care forms, police medical examination forms) often contain sections for health providers and police to fill out, it is important for both kinds of providers to be familiar with the forms, with how to fill them out properly, with their role in linking survivors to the police/health facility (depending on where the survivor presented first), and with working collaboratively to ensure completion of the forms in preparation for legal proceedings.

In Tanzanian refugee contexts, this intervention is being operationalized as follows:

- Locally-assembled PRC kits were put together by all health facilities.

- The PRC form was standardized for use in all camp locations

- Training on forensic evidence collection and accurate PF3 form completion was carried out with PRC health providers.

- The police were tasked with including a forensic police investigator in the review of a Memorandum of Understanding between UNHCR and the police; allocation of funds for forensic evidence processing in all locations; and procuring PRC kit items that were not already available within the health facilities.

- The Protection Unit was tasked with ensuring that relevant actors attend SGBV SWG monthly coordination meetings at camp level; contributing to procuring necessary equipment; and conducting awareness-raising sessions on forensic evidence practice.

- Six health providers have since been trained in forensic chain management by the healthcare focal point. Training topics included forensic evidence collection and storage, as well as PRC kit assembly procedures.

- A coordinated engagement among Ministry of Home Affairs, Police and Government Chemist in the regional 
analytical laboratory of Mwanza was conducted. Consensus was built on next steps for the intervention, including: plans for the government chemist to train health workers in forensic evidence chain management, and plans for the government to inspect health facilities for their preparedness for forensic chain management (collection tools, storage, etc.).

Since the commencement of the intervention, there has been an improvement in the filling of PF3 forms by police, although periodic refresher training sessions are recommended due to police staff transfers. PRC kits are now available in all health facilities in the participating camps, with periodic reporting and support from UNHCR and Health focal points. Improved coordination by the Protection SGBV Unit has caused improvements in referral mechanisms and pathways. Improvements have also been observed in the response time to survivors in various service delivery points. Monthly meetings convened by UNHCR with key actors tasked with taking stock of the intervention (IRC, health partners, government protection officers, police) has facilitated solutions to some of the challenges.

Partner(s):

International Rescue Committee

LVCT Health

Medical Teams International

Médecins Sans Frontières

Ministry of Home Affairs

Police Department

SGBV implementing partner

Tanzanian Red Cross

For further information, contact:

Benon Orach: orachb@unhcr.org

Rehema Katyega: katyega@unhcr.org

Dr.Yoel Mashimba:ymashimba@medicalteams.org

Miata Tubee Johnson: johnsonm@unhcr.org

Michael Gaitho: Michael.Gaitho@lvcthealth.org

\section{Promising Practice from Uganda: The 'Zero Tolerance Village Alliance'}

\footnotetext{
- he 'Zero Tolerance Village Alliance' (ZTVA) is a community-based SGBV prevention intervention that was developed for South African rural settings by the Thohoyandou Victim Empowerment Programme under the leadership of Fiona Nicholson, and introduced to refugee settings for the first time in Uganda. Rwamwanja settlement served as the pioneer refugee intervention site based on high reported rates of SGBV.
}

\section{The intervention involves the following components:}

Community Mapping and Dialogues: to identify all structures and agencies that can be targeted for participation, including providing SGBV services. Community dialogues are used to provide information about the intervention and promote community ownership.

Establishment of a Stakeholder Forum (SF): A SF is appointed and its membership is representative of community sectors (i.e., community leadership, churches, education, business). The SF holds monthly meetings to evaluate intervention implementation, and to ensure community members attend training workshops under the intervention.

Signing a Memorandum of Agreement (MoA): SF members sign a MoA to help formalize and guide the partnership between the SF and the implementing partner. The MoA outlines a list of criteria that the SF will help the community meet in order to be formally inducted into an alliance of villages that are branded as SGBV'zero tolerance zones.'

Training: Key community groups are sensitized on SGBV issues via training sessions. Leaflets summarizing the trainings are provided, and trainees are encouraged to independently train two other community members after receiving their own individual training.

Pledge-Taking Ceremony: Once the expectations outlined in the MoA were achieved by the village, the village receives sponsorship for a public ceremony, during which men in the community are invited to take a public pledge to proactively address the eradication of SGBV in their villages, and awards are issued to various community members for taking a stand against SGBV, or sharing their testimonies. 


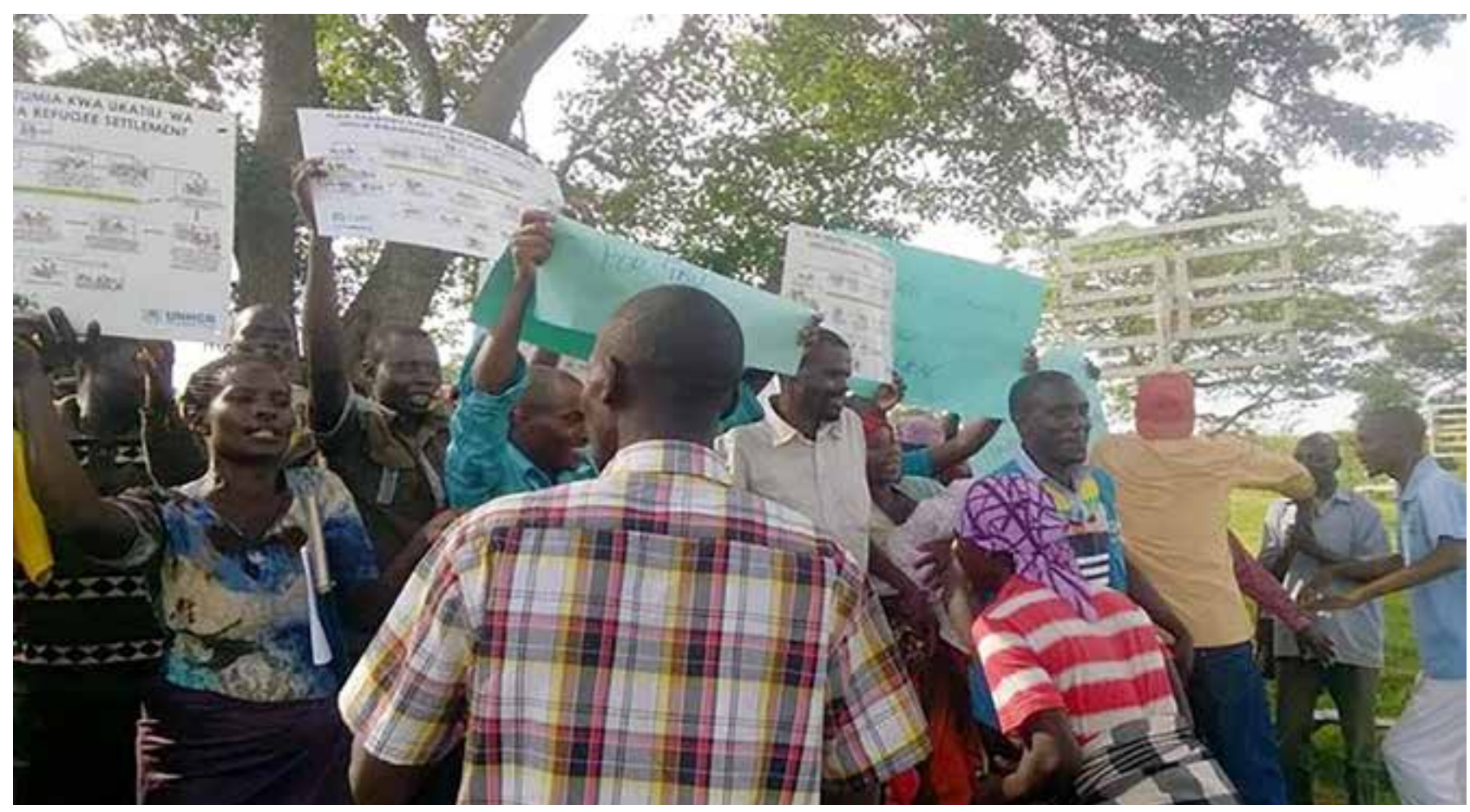

Alliance Identification: The pledge-taking ceremony culminates in the unveiling of a community billboard, indicating the name of the village and declaring their zero-tolerance status and induction into an 'alliance' of zero-tolerance villages. A small allowance was also made available for the villages to use in building a sense of community after the ceremony.

Evaluation results of this intervention in Ugandan refugee settings demonstrated a statistically significant reduction in the occurrence of: physical intimate partner violence (IPV) (for men and women), sexual IPV (for men), non-partner physical violence (for men and women), and non-partner sexual violence (for women).

To date, the ZTVA intervention has been implemented in about 15 refugee villages in Uganda. Background preparations (including training of implementing partners, sensitization of community leaders, and/or creation of Stakeholder Forums, etc.) have also been carried out to introduce the intervention in refugee settings in Djibouti, Ethiopia, and Zambia.

Partner(s):

Lutheran World Federation

Office of the Prime Minister

For further information, contact:

Mildred Ouma: ouma@unhcr.org

Monicah Rwotmon: monicah.rwotmon@lutheranworld.org

Fiona Nicholson: nicholsonfiona27@gmail.com

\section{Case Study from South Sudan: \\ Community-Based Approaches in Protection (SGBV Prevention, Risk Mitigation, Child Protection, and Youth Engagement)}

R efugees in Upper Nile State reside in four camps in Maban County, with heavy reliance on humanitarian agencies for the provision of life-saving and basic services. While significant progress has been made, concerns related to SGBV incidents, such as forced marriage, rape, FGM, and denial of opportunities to women and girls continue to be reported. In addition, the presence of armed elements has compromised the civilian and humanitarian character of asylum and contributed to the occurrence of some SGBV incidents.

A multi-faceted intervention was therefore developed with the objectives of: ensuring the safety of SGBV survivors in Maban refugee camps (including, but not limited to providing community-based safe shelters); enhancing social interaction, establishing or strengthening social networks, and building confidence in advocacy and information-sharing; and building the capacity of community-based structures in the timely identification, referral, and response to arising needs in the community. 
To effectively engage community-based structures in meeting these objectives, UNHCR, together with SGBV and Child Protection and Youth partners, has continued to enhance the capacity of SGBV advocates, SASA! Activists, refugee leaders, women and youth representatives, Community Activists, and representatives of Community-Based Safe Shelters to enhance their contribution toward prevention, mitigation and response to SGBV concerns in coordination with UNHCR and partners. Specific interventions include:

- The establishment of Community-Based Safe Shelters - a community-driven initiative - to ensure survivors are not exposed to risk.

- Women center activities (listening sessions for women and girls), which provide a safe platform for discussing issues affecting them and for sharing recommendations for addressing the issues.

- A 'whole society' engagement in SGBV response and prevention with the particular inclusion of youth and children in the spaces where they live, play, work and learn.

- A robust SGBV program entailing the SASA! methodology, a 'whole society,' community-based approach in which SASA! activists, Protection monitors, SGBV Advocates, Community Leaders, Association Representatives, and religious leaders are all engaged in awareness-raising and information dissemination on SGBV at village level.

- Deliberate engagement of boys and men in the SGBV program as catalysts for positive behavioral change.

- Case management by partner focal points ensuring a multi-sectoral response.

- Provision of tents and non-food items.

\section{Partner(s):}

Commission for Refugee Affairs

County authorities

Danish Refugee Council

Ministry of Gender, Child, and Social Welfare

Relief International

Save the Children International

\section{For further information, contact:}

SO Bunj AoR 
